\title{
Forced to cooperate
}

Citation for published version (APA):

Dupuis, L. (2018). Forced to cooperate: mechano-chemical interactions in cardiac sarcomeres. [Doctoral Thesis, Maastricht University]. Maastricht University. https://doi.org/10.26481/dis.20181109ld

Document status and date:

Published: 01/01/2018

DOI:

10.26481/dis.20181109ld

Document Version:

Publisher's PDF, also known as Version of record

\section{Please check the document version of this publication:}

- A submitted manuscript is the version of the article upon submission and before peer-review. There can be important differences between the submitted version and the official published version of record.

People interested in the research are advised to contact the author for the final version of the publication, or visit the DOI to the publisher's website.

- The final author version and the galley proof are versions of the publication after peer review.

- The final published version features the final layout of the paper including the volume, issue and page numbers.

Link to publication

\footnotetext{
General rights rights.

- You may freely distribute the URL identifying the publication in the public portal. please follow below link for the End User Agreement:

www.umlib.nl/taverne-license

Take down policy

If you believe that this document breaches copyright please contact us at:

repository@maastrichtuniversity.nl

providing details and we will investigate your claim.
}

Copyright and moral rights for the publications made accessible in the public portal are retained by the authors and/or other copyright owners and it is a condition of accessing publications that users recognise and abide by the legal requirements associated with these

- Users may download and print one copy of any publication from the public portal for the purpose of private study or research.

- You may not further distribute the material or use it for any profit-making activity or commercial gain

If the publication is distributed under the terms of Article $25 \mathrm{fa}$ of the Dutch Copyright Act, indicated by the "Taverne" license above, 


\section{Forced to क्रित्रि}

Mechano-Chemical Interactions in Cardiac Sarcomeres

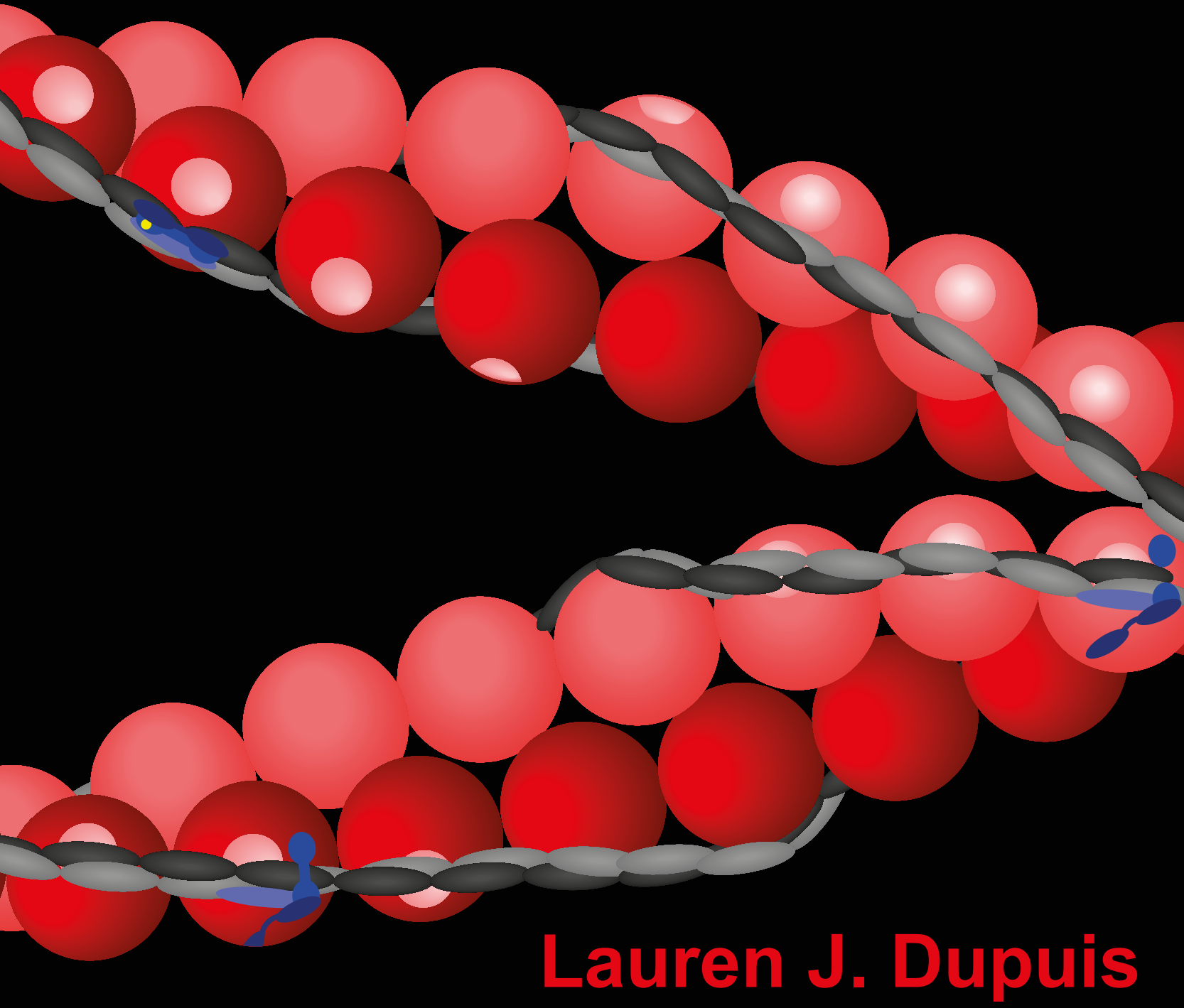





\section{Forced to Cooperate Mechano-Chemical Interactions in Cardiac Sarcomeres}

\section{DISSERTATION}

to obtain the degree of Doctor at the Maastricht University, on the authority of the Rector Magnificus

Prof.dr. Rianne M. Letschert,

in accordance with the decision of the Board of Deans,

to be defended in public

on Friday 9 November 2018 at 10:00am

by

Lauren J. Dupuis 


\section{Supervisor:}

Prof. dr. Tammo Delhaas

\section{Co-Supervisor:}

Dr. ir. Joost Lumens

\section{Assessment Committee:}

Prof. dr. Paul G.A. Volders (chairman)

Dr. ir. Peter H.M. Bovendeerd (Eindhoven University of Technology)

Prof. dr. ir. Chris T.A. Evelo

Prof. dr. ir. Ralf L.M. Peeters

Prof. dr. Jolanda van der Velden (VU Amsterdam)

Financial support for the publication of this thesis as provided by Maastricht University is gratefully acknowledged. 


\section{Contents}

Chapter 1

Introduction

Chapter 2

Mechano-chemical interactions in cardiac sarcomere contraction: A

computational modeling study

\section{Chapter 3}

High tension in sarcomeres hinders myocardial relaxation: a

computational study

\section{Chapter 4}

Mechano-chemical interactions in cardiac sarcomere shortening

\section{Chapter 5}

Linking cross-bridge cycling kinetics to response to cardiac

resynchronization therapy: A Multiscale modeling study

\section{Chapter 6}

General Discussion

Summary

Valorisation

Acknowledgements

Curriculum vitae and publications 



\section{Chapter 1}

\section{Introduction}

The heart is a component of the circulatory system and functions within a complex framework of mechanics, metabolism, and electrics. Within this complex framework, the rhythmic contraction and relaxation of cardiac muscle (myocardium) drives the circulation of blood throughout the body.

Since the work of Ringer (1), it has been widely known that calcium $\left(\mathrm{Ca}^{2+}\right)$ triggers contraction in cardiac muscle. Consecutive experiments by other researchers have shown that relatively small increases in $\left[\mathrm{Ca}^{2+}\right]$ results in disproportionately large increases in muscle force $(2,3)$. Donaldson and Kerrick (4) first suggested in their experiments on skeletal muscle in frog that the disproportionate increases in force were due to interacting sites along the muscle, implying cooperativity. Following their suggestion, it became common to represent the calcium-force relationship in muscle with a Hill-type sigmoidal curve (5). The original use for the Hill-type sigmoidal curve was to understand cooperative binding of oxygen within a single molecule of hemoglobin. However, because cooperative interactions in the sarcomere occur along a longer distance than a single molecule, the mechanism of cooperativity represented by the Hill model cannot fully explain cooperativity in the calcium-force relationship. Unfortunately, direct imaging of sub-cellular level processes responsible for cooperativity is impossible because these processes are on too small of a scale and occur too quickly. Although the technology to directly visualize the cooperativity mechanism in cardiac muscle may not be available yet, computational models can be developed and utilized to try to understand the interactions and push knowledge one step further than current technologies allow us to see. 


\section{General Aim}

In this thesis, we aim to build a computational model of sarcomere mechanics that is based on a novel hypothesis for the mechanism of cooperativity in cardiac muscle. We suggest that there is an intrinsic chemical cooperativity in the myocardium that is boosted by the mechanical tension in the muscle. We construct our model step-bystep from the ability to mimic the most basic static experiments towards the ability to mimic the shortening and relengthening of myocardial tissue that characterizes the rhythmic contraction and relaxation in the heart. Because the model is developed for eventual use in understanding clinically relevant problems in the human heart, we implement the newly developed sarcomere contraction model in a modeling framework of the circulation. As a first test, the combined model is applied to understand the relationship between cellular level mechanics and organ level hemodynamics in a heart with dyssynchronous activation patterns.

\section{Outline of Thesis}

Experiments have been performed in which cardiac muscle cells are skinned to allow control of the $\mathrm{Ca}^{2+}$ concentration $\left(\left[\mathrm{Ca}^{2+}\right]\right)$ in the intracellular space. In these experiments, $\left[\mathrm{Ca}^{2+}\right]$ is held constant and the steady-state isometric tension is measured $(2,3)$. We utilize these results for comparison to our work in Chapter 2 where we implement our novel hypothesis of cardiac cooperativity in a computational model of the sarcomere called the MechChem model of mechano-chemical interactions in the cardiac sarcomere. The MechChem model is tested with the conditions of static skinned muscle experiments (6). In addition, we present background on sarcomere physiology.

As a next step, we implement time dependence in the MechChem model. In a physiological situation, the free calcium concentration in the cytoplasm rises and falls in time. Relaxation begins when calcium concentration in the cytoplasm decreases due to extrusion of $\mathrm{Ca}^{2+}$ through various channels and pumps. Cardiomyocytes do not reach steady state tension because they must relax before the next heartbeat. While experiments have shown that greater peak tension in the thin filament results in 
prolongation of contraction during the isometric twitch $(7,8)$, the authors of the experiments do not offer an underlying mechanism to explain the prolongation of contraction. We implement a model of the $X B$ cycle in MechChem and use the model to try to explain the mechanism underlying the prolongation of contraction in sarcomeres under greater peak tension (Chapter 3). We hypothesize that the prolongation of contraction at higher sarcomere lengths is due to higher tension in the thin filament because this high tension strengthens the bond between $\mathrm{Ca}^{2+}$ and the troponin complex, rendering it energetically less favorable for $\mathrm{Ca}^{2+}$ to detach.

As a next step, we implement sarcomere shortening in the MechChem model as cardiac tissue shortens during contraction and re-lengthens during relaxation throughout a cardiac cycle. To understand the change in length of the myocardium, experiments can be performed on isolated cardiac muscle. In the experiments, the initial sarcomere length is constant, and the muscle contracts against various afterloads. The intracellular calcium transient rises and falls throughout contraction, and the tension generated by the muscle is recorded. These experiments are referred to as isotonic twitch experiments. In Chapter 4 we outline the implementation of the isotonic twitch in the MechChem model and compare the simulated results to the experimental data of Katsnelson et al. (9).

Understanding clinically relevant problems was the eventual reason for the development of the MechChem model. To this end, MechChem was implemented within the CircAdapt model of the human heart and closed-loop circulation. As a first test, the combined model of MechChem in CircAdapt was utilized to understand the relationship between cellular- and organ-level function in failing hearts. In the healthy human heart, the ventricular myocardium contracts and relaxes in a well-coordinated, synchronous manner, leading to a rather homogeneous distribution of work load across the ventricular walls. In patients with heart failure, however, this mechanical coordination is often lost due to the conduction disorder left bundle branch block (LBBB). LBBB is characterized by heart failure and delayed electrical activation of the left ventricular free wall relative to the septum and the right ventricular free wall 
leading to asynchronous contraction of the ventricular myocardium. A common treatment for LBBB is cardiac resynchronization therapy (CRT), which aims at restoring synchrony of ventricular activation through pacing of both ventricles. Unfortunately, a subset of patients does not respond positively to this treatment. It has been hypothesized that positive response to CRT depends on whether the underlying substrate is merely electrical or a combination of electrical and mechanical substrates (10). In Chapter 5, we tested the combined MechChem-CircAdapt model by simulating failing hearts with LBBB and while resynchronized. We compared tissue deformation patterns and response to CRT to the clinical data in humans as presented by Leenders et al. (11).

In our concluding chapter (Chapter 6) we present a general discussion where we look at the connection between the studies in Chapters $\mathbf{2}-\mathbf{5}$ as well as their possible implications to the broader field. 


\section{References}

1. Ringer S. A further Contribution regarding the influence of the different Constituents of the Blood on the Contraction of the Heart. The Journal of Physiology. 1883;4(1):29-42.

2. Dobesh DP, Konhilas JP, de Tombe PP. Cooperative activation in cardiac muscle: impact of sarcomere length. American Journal of Physiology - Heart and Circulatory Physiology. 2002;282(3):H1055H62.

3. Kentish JC, ter Keurs HE, Ricciardi L, Bucx JJ, Noble MI. Comparison between the sarcomere length-force relations of intact and skinned trabeculae from rat right ventricle. Influence of calcium concentrations on these relations. Circulation Research. 1986;58(6):755-68.

4. Donaldson SK, Kerrick WG. Characterization of the effects of Mg2+ on Ca2+- and Sr2+-activated tension generation of skinned skeletal muscle fibers. The Journal of General Physiology. 1975;66(4):427-44.

5. Hill AV. The Combinations of Haemoglobin with Oxygen and with Carbon Monoxide. I. Biochemical Journal. 1913;7(5):471-80.

6. Dupuis LJ, Lumens J, Arts T, Delhaas T. Mechano-chemical Interactions in Cardiac Sarcomere Contraction: A Computational Modeling Study. PLOS Computational Biology. 2016;12(10):e1005126.

7. Janssen PM, Hunter WC. Force, not sarcomere length, correlates with prolongation of isosarcometric contraction. American Journal of Physiology. 1995;269(2):H676-H85.

8. Kassiri Z, Myers R, Kaprielian R, Banijamali HS, Backx PH. Rate-dependent changes of twitch force duration in rat cardiac trabeculae: a property of the contractile system. The Journal of Physiology. 2000;524(Pt 1):221-31.

9. Katsnelson LB, Nikitina LV, Chemla D, Solovyova O, Coirault C, Lecarpentier Y, et al. Influence of viscosity on myocardium mechanical activity: a mathematical model. Journal of Theoretical Biology. 2004;230(3):385-405.

10. Lumens J, Tayal B, Walmsley J, Delgado-Montero A, Huntjens PR, Schwartzman D, et al. Differentiating Electromechanical From Non-Electrical Substrates of Mechanical Discoordination to Identify Responders to Cardiac Resynchronization Therapy. Circulation: Cardiovascular Imaging. 2015;8(9).

11. Leenders GE, Lumens J, Cramer MJ, De Boeck BWL, Doevendans PA, Delhaas T, et al. Septal Deformation Patterns Delineate Mechanical Dyssynchrony and Regional Differences in Contractility: Analysis of Patient Data Using a Computer Model. Circulation Heart Failure. 2012;5(1):87-96. 



\title{
Chapter 2
}

\section{Mechano-chemical interactions in cardiac sarcomere contraction: A computational modeling study}

This chapter is based on:

Lauren J. Dupuis, Joost Lumens, Theo Arts, and Tammo Delhaas (2016). Mechanochemical interactions in cardiac sarcomere contraction: A computational modeling study. In PLoS Computational Biology. Volume 12, issue 10.

\begin{abstract}
We developed a model of cardiac sarcomere contraction to study the calcium-tension relationship in cardiac muscle. Calcium mediates cardiac contraction through its interactions with troponin $(T n)$ and subsequently tropomyosin molecules. Experimental studies have shown that a slight increase in intracellular calcium concentration leads to a rapid increase in sarcomeric tension. Though it is widely accepted that the rapid increase is not possible without the concept of cooperativity, the mechanism is debated. We use the hypothesis that there exists a base level of cooperativity intrinsic to the thin filament that is boosted by mechanical tension, i.e. a high level of mechanical tension in the thin filament impedes the unbinding of calcium from $\mathrm{Tn}$. To test these hypotheses, we developed a computational model in which a set of three parameters and inputs of calcium concentration and sarcomere length
\end{abstract}


result in output tension. Tension as simulated appeared in good agreement with experimentally measured tension. Our results support the hypothesis that high tension in the thin filament impedes $T n$ deactivation by increasing the energy required to detach calcium from the $T n$. Given this hypothesis, the model predicted that the areas with highest tension, i.e. closest to the Z-disk end of the single overlap region, show the largest concentration of active $T n^{\prime}$ s.

\section{Introduction}

It has been widely known since the 1883 work of Ringer [1] that calcium ions $\left(\mathrm{Ca}^{2+}\right)$ regulate muscle contraction. Although the mechanism was unknown at the time, Ringer found that rat hearts did not contract forcefully unless $\mathrm{Ca}^{2+}$ was added to the solution perfusing the muscle. Since Ringer's discovery the relationship between $\mathrm{Ca}^{2+}$ concentration $\left(\left[\mathrm{Ca}^{2+}\right]\right)$ and tension in muscle has been widely studied. Experiments have shown a steep rise in steady state tension with relatively little increase in intracellular $\left[\mathrm{Ca}^{2+}\right]$. The desire to understand the steepness of the $\left[\mathrm{Ca}^{2+}\right]-$ tension relationship has led to the hypothesis that cardiac muscle contraction is highly cooperative $[2,3]$. Cooperativity implies that a single event encourages subsequent similar events. In the case of muscle contraction, a single $\mathrm{Ca}^{2+}$ binding invoking the generation of tension might enhance additional $\mathrm{Ca}^{2+}$ bindings to generate more tension [4]. Various hypotheses about the mechanism of cooperativity in cardiac muscle have been proposed.

Before discussing cooperativity, we explain the structures and molecular mechanisms involved in cardiac contraction. The cardiac muscle is striated, meaning that it is composed of a repeating organization of contractile units known as sarcomeres (Figure 2.1A). Within the sarcomeres thick and thin filaments can interact with one another to generate tension $[5,6]$.

The sarcomere is bounded by two opposing Z-disks with the thin filaments protruding from each (Figure 2.1A). A thin filament contains a double helical arrangement of spherical actin monomers (Figure 2.1B), each with a myosin binding site on its surface 
facing the outside of the helix [7]. A thin filament is surrounded by thick filaments, each having myosin heads protruding every $14.3 \mathrm{~nm}$ that can bind to actin to form a cross-bridge $(X B)$ that can generate force [8]. The thick and thin filaments range in length from $\sim 1.5$ to $\sim 1.7 \mu \mathrm{m}$ and $\sim 1.1$ to $1.3 \mu \mathrm{m}$, respectively [9]. The distance between Z-disks is equal to the sarcomere length $(S L)$. Opposing thin filaments can mutually overlap in the so-called double overlap region (Figure 2.1A). The single overlap region is the section of the thick filament outside of the double overlap region that overlaps with the thin filament. We assume that $X B^{\prime}$ 's can form solely in the single overlap region [10]. As $S L$ increases up to about $2.3 \mu \mathrm{m}$, the length of the double overlap region decreases, thereby lengthening the single overlap region, thus providing more opportunities for $X B$ formation and subsequently higher force generation (Figure 2.1A).

$\mathrm{Ca}^{2+}$ activates the thin filament, allowing $X B^{\prime} s$ to form and force to develop (Figure 2.1B). Seven actin monomers, one tropomyosin $(T m)$ molecule, and one troponin complex $(T n)$ form a repeating structure called a regulatory unit $(R U)$ along the thin filament (Figure 2.2A) [11]. Tm molecules are double helical molecules that follow the same helical path as the actin monomers, overlapping one another head to tail under the $T n$. At rest, the $T m$ molecule wraps around the actin monomers in a position that blocks the myosin binding sites, thereby hindering the formation of $X B^{\prime} s$ and thus contraction. Each $T n$ is composed of three subunits, the inhibitory subunit $(T n l)$, the $\mathrm{Tm}$ binding subunit ( $\mathrm{TnT}$ ) that attaches the $\mathrm{Tn}$ to $\mathrm{Tm}$, and the $\mathrm{Ca}^{2+}$ binding subunit (TnC) [12](Figure 2.2B). Tnl binds to actin monomers at rest and essentially anchors the Tm molecule in place, blocking the binding sites on actin [13]. When the cell depolarizes, $\mathrm{Ca}^{2+}$ enters and may bind to $\mathrm{TnC}$ opening a hydrophobic patch on the $\mathrm{N}$ terminus of $\mathrm{TnC}$. The $\mathrm{COOH}$ terminus of $\mathrm{Tnl}$ exhibits a strong affinity for the $\mathrm{NH}_{2}$ terminus opened on $\mathrm{TnC}$ and binds strongly to it, and the inhibitory peptide of $\mathrm{TnI}$ releases actin[14]. The $T m$ strand moves laterally, thus freeing nearby $X B$ binding sites on the thin filament (Figure 2.2C). We refer to the latter movement of $\mathrm{Tm}$ as the activation of the $\mathrm{Tn}$ (Figure 2.2B). It has been shown that, along with $\mathrm{Ca}^{2+}$ binding to 
$T n C$, the formation of a strong $X B$ is necessary to fully unblock the binding sites on actin [15]. During relaxation the $\left[\mathrm{Ca}^{2+}\right]$ in the cell decreases, $\mathrm{TnC}$ releases the bound $\mathrm{Ca}^{2+}$ ions, and $\mathrm{Tnl}$ rebinds to actin moving the $\mathrm{Tm}$ back over the $\mathrm{XB}$-binding sites preventing further force generation [16].

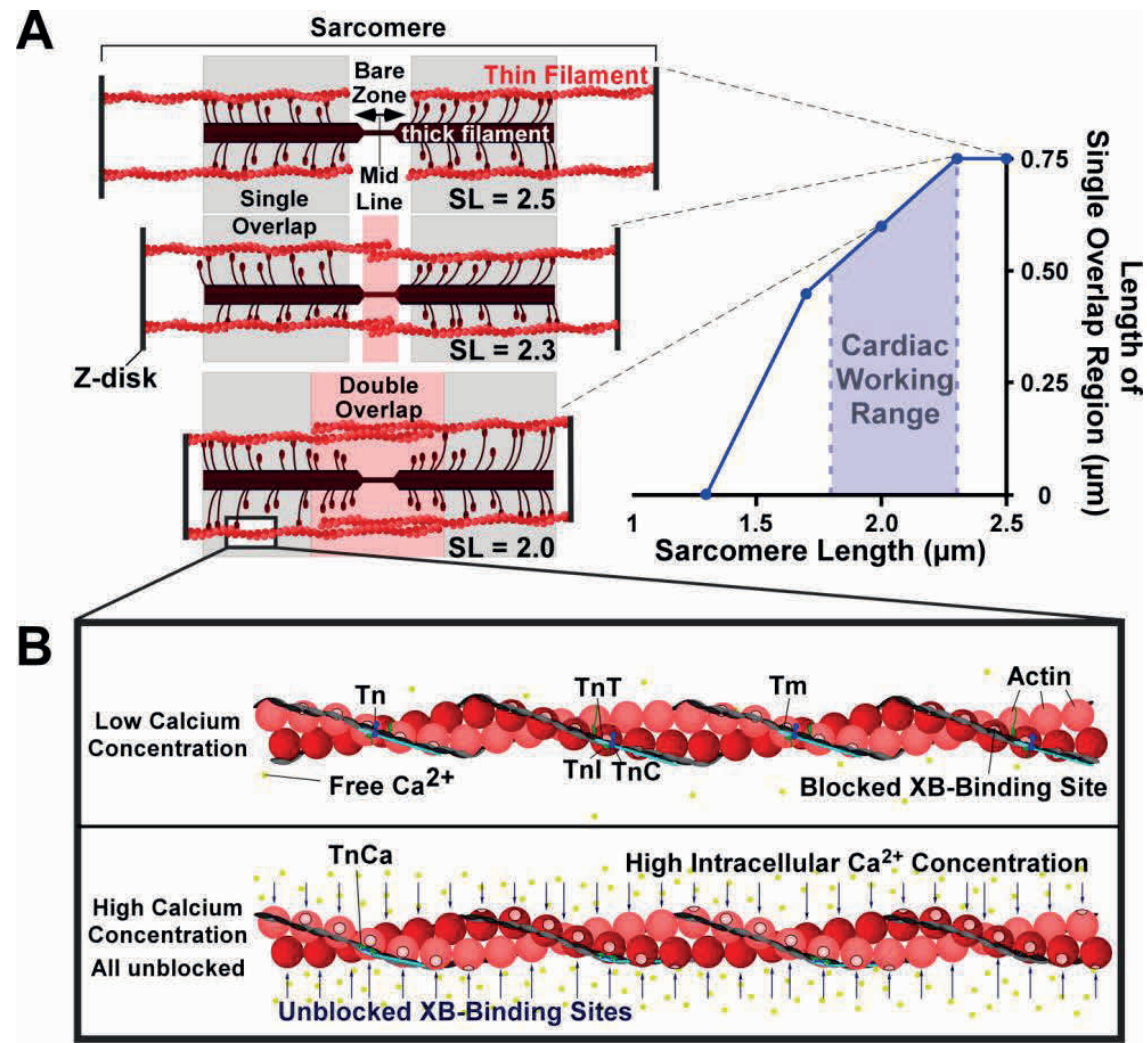

Figure 2.1: Schematic Representation of Sarcomere. (A). Sarcomeres are composed of thick and thin filaments that interact to generate force. Sarcomere length $(S L)$ determines the length of single overlap region where cross-bridges $(X B)$ can form and generate force. (B) At rest, the binding sites on the spherical actin are blocked by a strand of tropomyosin $(T m)$. Ca binding to $T n(T n C a)$ activates a troponin complex $(T n)$ resulting in the lateral movement of the Tm strand that unblocks the $X B$-binding sites. The $T n$ is composed of three subunits, troponin $\mathrm{C}(\operatorname{TnC})$, troponin I $(T n l)$, and troponin $\mathrm{T}(\operatorname{Tn} T)$. 
Commonly the relation between intracellular $\left[\mathrm{Ca}^{2+}\right]$ and muscle tension is described by the Hill-relation, having zero active tension at low $\left[\mathrm{Ca}^{2+}\right]$ and approaching a level of saturation at high $\left[\mathrm{Ca}^{2+}\right.$. If a single $\mathrm{Ca}^{2+}$ ion would bind to a single $\mathrm{Tn}$ complex without cooperativity, for a physiological muscle contraction, the required increase in $\left[\mathrm{Ca}^{2+}\right]$ would be unphysiologically large. By comparing the relatively small, physiological change in $\left[\mathrm{Ca}^{2+}\right]$ with the resulting large change in muscle tension, $\mathrm{Ca}^{2+}$ binding to $\mathrm{Tn}$ requires an increase of cooperativity by the so-called Hill coefficient $n$. In experiments by Dobesh et al. [18] it is found $n \approx 7$.

On the basis of conventional chemical principles, such a high level of cooperativity requires full cooperation between a number of binding sites at least equal to the Hill coefficient. In cardiac muscle, each $\mathrm{Tn}$ contains three binding sites for $\mathrm{Ca}^{2+}$. Each of the two intertwined actin strands in the thin filament contains a Tn after every sequence of seven actin monomers. The $T n^{\prime}$ s of both actin strands located at equal lengths form repetitive pairs of $T n^{\prime}$ s, or in-register $T n^{\prime}$ s. Galińska-Rakoczy et al. showed that the Cterminal section of $\mathrm{Tnl}$ extends over the actin monomer on the adjacent strand reaching to the $\mathrm{Tm}$ molecule [17]. Their results suggest that upon $\mathrm{Ca}^{2+}$ binding the $\mathrm{Tn}$ may influence the position of the $T m$ molecule on the surface of the adjacent actin strand as well. Because of this interaction, we consider some degree of cooperativity of $\mathrm{Ca}^{2+}$ binding to in-register $\mathrm{Tn}^{\prime}$ s. Each $\mathrm{Tn}$ complex has 3 binding sites for $\mathrm{Ca}^{2+}$, with one of them having a substantially lower affinity for $\mathrm{Ca}^{2+}$ than the others [19]. So, potentially, each pair of in-register Tn complexes contains 6 binding sites, limiting the intrinsic Hill coefficient to a maximum value of 6 if each of the binding sites exhibits an equal degree of cooperativity. The term 'intrinsic' is used to indicate that the binding is considered purely chemical, excluding effects of mechanical tension. In experiments by Sun et al. [20], in the absence of muscle tension, $\mathrm{Ca}^{2+}$ was bound to $\mathrm{Tn}$ with a Hill coefficient of 3 , indicating that the intrinsic cooperativity was about half of the abovementioned maximum. Even in the case of full cooperativity between in-register $T n^{\prime}$ s, i.e. with a intrinsic cooperativity coefficient equaling 6 , one more binding site on a $T n$ in the next $R U$ would be necessary to reach a cooperativity represented by the 
Hill coefficient of 7 in experiments of Dobesh et al. [18]. However, $T n^{\prime}$ s are separated along the thin filament $T n^{\prime}$ s by about $35 \mathrm{~nm}$, a distance too large for conventional mechanisms of chemical cooperativity.

In many current sarcomere models, the trend is to represent cooperativity along the thin filament as the interaction between neighboring $X B^{\prime} S$ and $R U^{\prime}$ s. Razumova and colleagues [21] developed a stiffness-distortion model of the $X B$ and tested three different models of nearest neighbor cooperative interactions ( $R U-R U, X B-X B, X B-R U)$. They found that while none of these interactions were able to completely account for the cooperative force development observed in experiments, each mechanism had a large impact on the $\left[\mathrm{Ca}^{2+}\right]$-tension relationship. $X B-X B$ interactions had the highest impact on peak force, $R U-R U$ interactions had the greatest impact on the steepness of the $\left[\mathrm{Ca}^{2+}\right]$-tension relationship, and $X B-R U$ interactions had the greatest impact on $\mathrm{Ca}^{2+}$ sensitivity. The model as developed by Rice and colleagues [22] was based on the model of Razumova and colleagues [21] including assumed nearest neighbor cooperativity. Campbell and colleagues [23] have developed a model of cooperativity in which adjacent $T m$ molecules overlap one another at the $T n$, thereby encouraging $R U$ activation when the nearest neighbor $R U$ is activated. It is not clear yet on what physical principles such $R U-R U, R U-X B$ and $X B-X B$ interactions would work.

It has further been proposed that at longer $S L^{\prime} S$ the constant volume of the myofiber lattice causes the filaments to squeeze closer together, possibly encouraging binding events by moving a myosin head within reach of a binding site on the thin filament $[24,25]$. Similarly, Daniel et al. propose that the cooperative realignment of binding sites occurs when the thin filament is strained by up to $2 \mathrm{~nm}$ [26], a lateral movement that could possibly move a binding site within reach of a myosin head. Still others believe that the end-to-end interactions of the $T m^{\prime}$ 's along the thin filament are responsible for the cooperative activation [27]. 

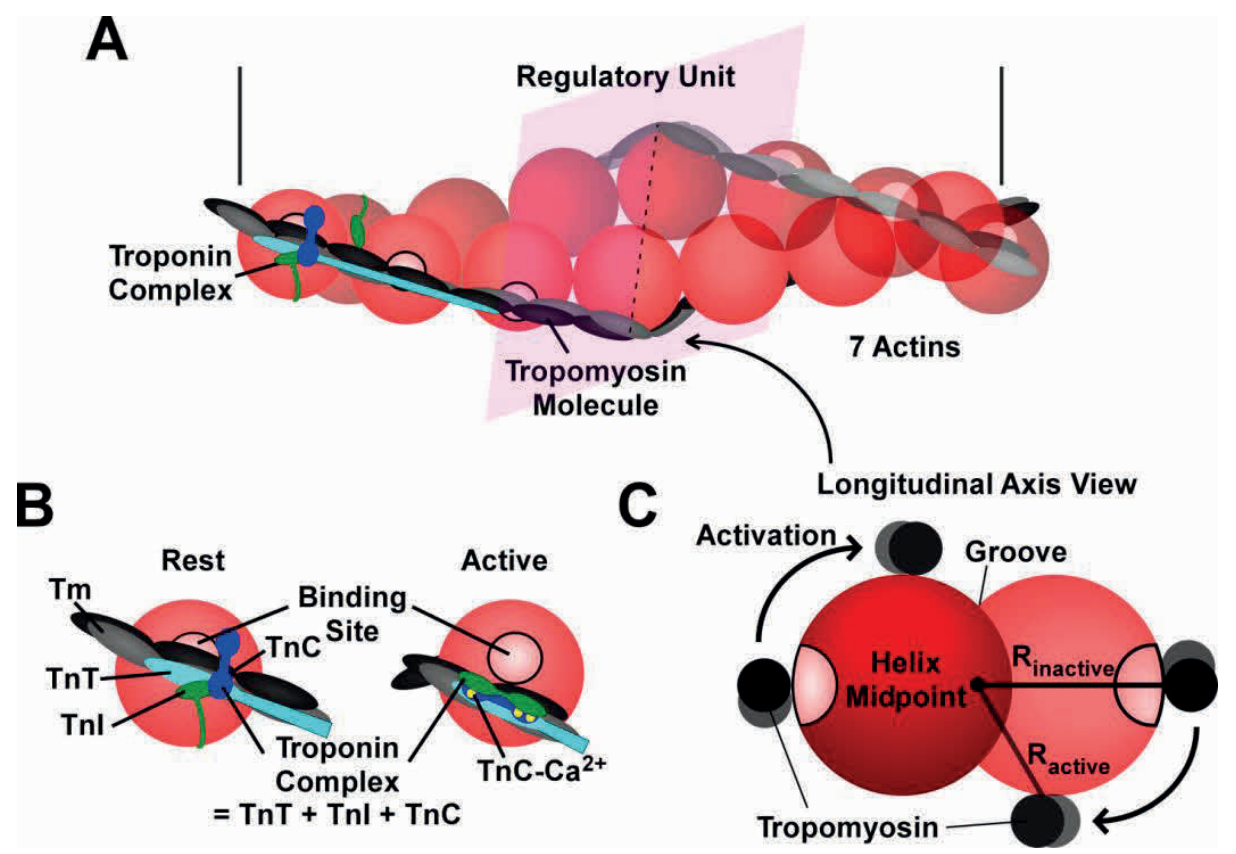

Figure 2.2: Schematic Representation of $T n$ activation. (A) $A R U$ is a functional unit of the sarcomere that is considered to work together. It is composed of 7 actin monomers, one tropomyosin $(T m)$ molecule, and one troponin complex $(T n)$. At rest, the C-terminal section of $T n l$ extends over the actin monomer on the adjacent strand reaching to the Tm molecule, hindering its movement [17]. That hindrance is removed upon the conformational changes resulting from $\mathrm{Ca}^{2+}$ binding to that $\mathrm{Tn}$. Note: $\mathrm{Tn}^{\prime}$ s on adjacent actin strands are located in-register with one another, but this is not displayed in the figure. (B) A conformational change in the $T n$ caused by the binding of calcium to $T n C$ effectively removes the $T m$ from the blocking position. (C) The thin filament in $(\mathrm{A})$ is viewed from the longitudinal axis. The movement of $\mathrm{Tm}$ from the position blocking the $X B$-binding sites toward the groove reduces the radius $(R)$ from the center of the actin double helix.

In the present study we introduce a mechanism based on the mechanochemical interaction of $\mathrm{Ca}^{2+}$ binding to $\mathrm{Tn}$. Binding of $\mathrm{Ca}^{2+}$ to $\mathrm{Tn}$ results in conformational changes in the Tn complex. We postulate that mechanical stretch of the thin filament facilitates the change in conformation by reducing the energetic increment required for binding of $\mathrm{Ca}^{2+}$ to $\mathrm{Tn}$. Thus, mechanical tension in the thin filament will shift the equilibrium of $\mathrm{Ca}^{2+}$ binding to $\mathrm{Tn}$ towards the bound state. The latter shift in equilibrium is quantified by the use of the general physical principle that the ratio of prevalence of two states depends on the difference in energy between the two states. 
We think that such a mechanism shows properties of cooperativity even though it differs from a pure chemical mechanism.

On the basis of the abovementioned mechanochemical principle, we designed a computational model of cardiac sarcomere mechanics that we call the MechChem model. The MechChem model is intended to predict the tension in a thin filament as a function of $\left[\mathrm{Ca}^{2+}\right]$ and sarcomere length. We investigated whether the mechanochemical mechanism can boost cooperativity from the intrinsic level with a Hill coefficient of 3 described by Sun et al. [20] up to the level of 7 shown by Dobesh et al. [18] in skinned muscle preparations. The energy to bind $\mathrm{Ca}^{2+}$ to $\mathrm{Tn}$ is assumed to decrease linearly with thin filament tension. Currently we focus on the static behavior, implying that thin filament tension was calculated while maintaining constant sarcomere length and $\left[\mathrm{Ca}^{2+}\right]$. Under static conditions we considered the chemical reactions involved to be in equilibrium.

In investigating the static behavior of cardiac muscle contraction, muscle tension as simulated with the MechChem model is compared with simulations based on conventional chemical binding of $\mathrm{Ca}^{2+}$ to $\mathrm{Tn}$ with a high degree of cooperativity, resulting in the typical S-shaped Hill curve. Experimental results to compare with were obtained from skinned muscle preparations, subject to various static levels of $\left[\mathrm{Ca}^{2+}\right]$ and sarcomere length as reported by Dobesh et al [18]. When fitting both the MechChem model and Hill-type model to experimental findings, a $\left[\mathrm{Ca}^{2+}\right]$-tension relation is found for each sarcomere length, requiring the estimation of 3 parameters. Furthermore, it is investigated which parameters values could remain while changing sarcomere length.

\section{Methods}

\section{Model Design}

Formation of $X B^{\prime}$ s is initiated by the binding of $\mathrm{Ca}^{2+}$ to $\mathrm{TnC}$ which we will refer to as the binding of $\mathrm{Ca}^{2+}$ to a in-register pair of $\mathrm{Tn}$, referred to in the equations as $\mathrm{Tn}_{2}$. The related chemical equilibrium reaction is represented by 


$$
\mathrm{Tn}_{2}+n \mathrm{Ca}^{2+} \leftrightarrows \mathrm{Tn}_{2} \mathrm{Ca}_{n}
$$

Parameter $n$ indicates the coefficient related to the intrinsic cooperativity of $\mathrm{Ca}^{2+}$ binding to $\mathrm{Tn}_{2}$. When $\mathrm{Ca}^{2+}$ binds to $\mathrm{Tn}, \mathrm{Tm}$ moves away from the position in which it blocks the $X B$-binding sites on the actin monomers. We assume that the unblocking of $X B$-binding sites, or activation of a $T n$, automatically implies $X B$ formation in the environment of that $T n$. After formation, the $X B$ exerts a longitudinal force $F_{X B}$ on the thin filament which is guided as tension along the thin filament to the Z-disk (Figure 2.3). Thus, toward the Z-disk, the longitudinal tension $S(x)$ in the thin filament is increasing by a step $F_{X B}$ at each location where a $X B$ has formed. Variable $x$ represents the distance from the beginning of the single overlap region moving toward the Z-disk (Figure 2.3). The maximum value $\left(x_{\max }\right)$ of $x$ represents the location on the thin filament where the single overlap region ends. The single overlap length depends on $S L$ and the assumed filament lengths as shown in S1 Appendix.

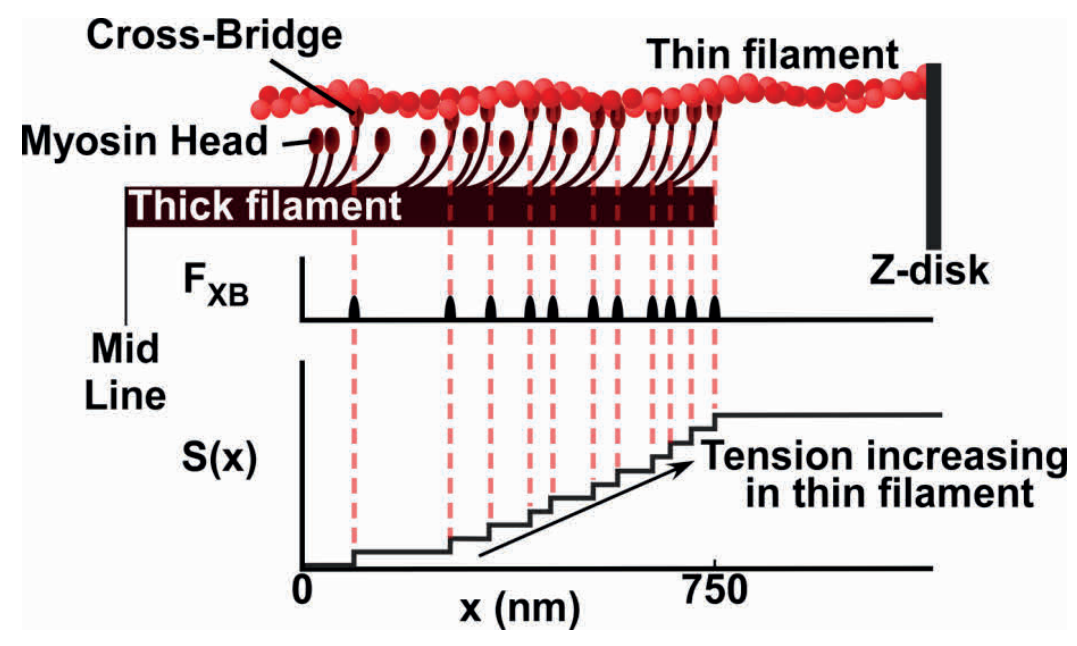

Figure 2.3: Build-up of tension in the thin filament. Each cross-bridge $(X B)$ generates an individual force $F_{X B}$. Tension $S(x)$ at location $x$ is the sum of each individual $X B$ force from the start of the single overlap region closest to the mid line $(x=0)$ to the position $x$. So, tension along the thin filament increases with each $X B$ exerting force. 
Looking from the middle of the sarcomere towards the Z-disk, for tension $S\left(x_{j}\right)$ just distal to $X B_{j}$ at position $x_{j}$ it holds:

$$
S\left(x_{j}\right)=\sum_{i=1}^{i=j} F_{X B, i} \text { with } 1 \leq j \leq j_{\max }
$$

The symbol $j_{\max }$ indicates the number of $X B^{\prime} s$ attached to the thin filament in the single overlap region. Equation 2.2 is graphically elucidated in Figure 2.3. Tension $S(x)$ increases with each attached $X B$ until distance $x$ exceeds the end of the single overlap region where no $X B^{\prime}$ 's can be formed. The total tension of the thin filament equals the tension acting on the Z-disk from that thin filament.

\section{Implementation of the MechChem model}

The following assumptions are key to the MechChem model implementation presented in this section:

- $\quad \mathrm{Ca}^{2+}$ binding to $\mathrm{Tn}$ moves $\mathrm{Tm}$ azimuthally on the thin filament, unblocking the $X B$-binding sites on nearby actin monomers.

- $\quad$ Unblocking of binding sites implies $X B$ binding and force development.

- $\quad X B^{\prime}$ s can form solely in the single overlap region.

- The total tension in the thin filament at the Z-disk is the sum of the individual $X B$ force acting on the thin filament.

- Tension hinders the deactivation of the thin filament. The energy required for $\mathrm{Tn}$ to release $\mathrm{Ca}^{2+}$ increases linearly with the mechanical tension in the thin filament.

- Binding of $\mathrm{Ca}^{2+}$ to an in-register $\mathrm{Tn}$ pair $\left(T n_{2}\right)$ is intrinsically cooperative, characterized by the base cooperativity coefficient $n$ (according to [20]: $n=3$ ).

- When simulating isometric tension development, all $X B^{\prime}$ 's generate equal force.

Referring to Equation 2.2, the principle of the model is explained by the existence of discrete locations where $X B^{\prime}$ s can form. In living muscle at any given time during 
contraction, there are countless $X B^{\prime}$ s. Thus, we decided to represent muscular force generation by the average of many discrete states, allowing us to describe tensions as continuous functions of distance $x$ along the representative thin filament. Focusing on the static conditions only, the involved chemical reactions are considered in equilibrium. According to Equation 2.1, we consider binding of $\mathrm{Ca}^{2+}$ to $\mathrm{Tn}$ as a chemical binding to an in-register $T n$ pair, named $T n_{2}$.

Equilibrium concentrations of $\mathrm{Ca}^{2+}, \mathrm{Tn}_{2}$, and $\mathrm{Tn}_{2} \mathrm{Ca}_{n}$ are related by:

$$
K_{\text {TnCa }}{ }^{n}=\frac{\left[\mathrm{Tn}_{2}\right]\left[\mathrm{Ca}^{2+}\right]^{n}}{\left[\mathrm{Tn}_{2} C a_{n}\right]}
$$

The symbol $K_{\text {TnCa }}$ represents the equilibrium constant, and $n$ is the base cooperativity coefficient representing the intrinsic cooperativity of $\mathrm{Ca}^{2+}$ binding to $\mathrm{Tn}_{2}$. We assume that when $\mathrm{Ca}^{2+}$ binds to the $\mathrm{Tn}_{2}$, the nearby $X B$-binding sites are unblocked. For the proportion of activated $T n^{\prime} s, P(x)$, at position $x$ along the thin filament, it holds

$$
P(x)=\frac{\left[T n_{2} C a_{n}\right](x)}{\left[T n_{2}\right](x)+\left[T n_{2} C a_{n}\right](x)}
$$

Solving Equation 2.3 for $\left[\mathrm{Tn}_{2} \mathrm{Ca}_{n}\right]$ and substituting the result into Equation 2.4 renders an expression for $P(x)$ as a function of $\left[\mathrm{Ca}^{2+}\right]$ and $K_{T n C a}$ (Equation 2.5).

$$
P(x)=\frac{1}{1+\left(\frac{K_{T n C a}(x)}{\left[C a^{2+}\right]}\right)^{n}}
$$

Because in the MechChem model binding of $\mathrm{Ca}^{2+}$ to $\mathrm{Tn}_{2}$ is assumed to depend on mechanical tension in the thin filament, the equilibrium constant $K_{\text {TnCa }}$ is considered to depend on the position $x$ along the thin filament. The energy required to detach $\mathrm{Ca}^{2+}$ from the $T n$ is assumed to increase linearly with the tension $S(x)$ in the thin filament. Thus, the equilibrium constant is multiplied by the exponential of the product of tension $S(x)$ and a constant $C_{S}$, representing the effect of the added affinity for $\mathrm{Ca}^{2+}$ by an increase of tension. The latter relation is based on the general physical principle 
that in equilibrium the ratio of state concentrations is proportional to the exponential of a constant multiplied by the energy difference between both states. Analogously, the ratio of ion concentrations on both sides of a membrane depends on the voltage difference across the membrane. So, we express the dependence of the equilibrium concentration constant, $K_{\text {TnCa }}$ on tension $S(x)$ by:

$$
K_{\text {TnCa }}(x)=K_{\text {TnCa0 }} e^{-C_{S} S(x)}
$$

The symbol $K_{\text {TnCaO }}$ represents the equilibrium constant in the absence of tension in the thin filament. The physical dimension of constant $C_{S}$ is the inverse of tension. Replacing $K_{T n C a}(x)$ in Equation 2.5 with Equation 2.6 yields the tension-dependent expression for $P(x)$ in Equation 2.7.

$$
P(x)=\frac{1}{1+e^{n\left(-C_{S} S(x)-\ln \left(\frac{\left[C a^{2+}\right]}{K_{\text {TnCa0 }}}\right)\right)}}
$$

We assume that the density of attached $X B^{\prime}$ s is proportional to $P(x)$. Assuming that all attached $X B^{\prime}$ s exert the same force during steady state isometric contraction, we find that the $X B$-induced force density $f_{\mathrm{XB}}(x)$ acting at location $x$ is proportional to $P(x)$ (Equation 2.8).

$$
f_{X B}(x)=C_{f} P(x)
$$

Constant $C_{f}$ represents force density with full Tn activation, having the physical dimension of force per unit length along the thin filament. After elimination of $P(x)$ by substitution of Equation 2.7 into Equation 2.8 and using the property that the derivative $\mathrm{d} S(x) / \mathrm{d} x$ of tension with respect to $x$ equals force density $f_{\mathrm{XB}}(x)$, we find the following differential equation for $S(x)$ with boundary condition $S(0)=0$ :

$$
\frac{d S(x)}{d x}=\frac{C_{f}}{1+e^{n\left(-C_{S} S(x)-\ln \left(\frac{\left[C a^{2+}\right]}{K_{\text {TnCa0 }}}\right)\right)}}
$$


Assuming $n=3$ according to the findings by Sun et al. [20] for the intrinsic thin filament cooperativity, Equation 2.9 contains 3 independent parameters, i.e., $C_{f}, C_{s}$ and $K_{T n C a O}$.

We have fitted the Hill-type model with the experimental data of Dobesh et al. [18] for comparison, expressing tension $S_{H}$ as a function of $\left[\mathrm{Ca}^{2+}\right]$. The three parameters $E C_{50}$, $n_{H}$, and $S_{\text {max }}$, represent the $\left[\mathrm{Ca}^{2+}\right]$ at the $50 \%$ level of maximum tension, the Hill coefficient, and maximum tension, respectively. Thus we used

$$
S_{H}=S_{\max } \frac{\left[\mathrm{Ca}^{2+}\right]^{n_{H}}}{E C_{50}^{n_{H}}+\left[\mathrm{Ca}^{2+}\right]^{n_{H}}}
$$

\section{Simulation Protocol}

Under normal conditions, cardiac muscle cells are enclosed by membranes that regulate the influx of $\mathrm{Ca}^{2+}$ ions from the extracellular space through channels and pumps [28]. Submersing the muscle in detergent causes perforations in the cellular membranes, a procedure known as muscle skinning. Due to these perforations, the membrane channels and pumps no longer regulate intracellular ion concentrations. Thus, it is assumed that when a skinned muscle cell preparation is submerged in a solution containing ions, the intracellular ion concentrations are equal to that of the immersing solution. Thus, the intracellular $\left[\mathrm{Ca}^{2+}\right]$ can be manipulated, and the muscle will contract and generate tension in response.

Data points published by Dobesh and colleagues (Figure 2A of the original article) [18] provided the experimentally measured tension values $\left(S_{\text {exp }}\right)$ at various $S L^{\prime} s$ and $\left[\mathrm{Ca}^{2+}\right]^{\prime}$ 's. We tested our model at a range of $\left[\mathrm{Ca}^{2+}\right]^{\prime}$ s between 0.001 and $10 \mu \mathrm{M}$ for five $S L^{\prime} \mathrm{s}$ ranging from 1.85 to $2.25 \mu \mathrm{m}$. Single overlap length $x_{\max }$ increases with SL according to the formulation of Rice et al [22], the equations of which are presented in the S1 Appendix (Equation 2.A1-2.A3). The parameters $C_{s}, C_{f}$ and $K_{\text {CaTno }}$ were varied so that the sum of the squared differences between the experimental data by Dobesh and the solution of the differential equation (Equation 2.9) was minimal. The minimization was performed on each individual curve. The Hill-type model with parameters $E C_{50}, S_{\max }$, 
and $n_{H}$ was fitted to the same experimental data. For the MechChem and Hill-type models, the residual errors were assessed to find systematic differences between model and measurement. Additionally, the root mean squared error (RMSE) was calculated for all individual curves (Equation 2.11). $S_{\text {model }}$ and $S_{\text {Dobesh }}$ represent the tension generated in the model and the tension measured experimentally by Dobesh et al., respectively. Because the tension values reported by Dobesh et al. referred solely to the active tension generated, the results of the MechChem model are also reported as active tension. Additionally, we calculate the tension solely in the thin filament. Hence, the passive tension component contributed by the extracellular matrix or titin is viewed as a separate component that would be additional to the calculated active tension. The number of points is represented by $j$.

$$
R M S E=\sqrt{\frac{\sum_{i=1}^{j}\left(S_{\text {model }}-S_{\text {Dobesh }}\right)^{2}}{j}}
$$

The RMSE compares the results of our model to the data from the experiments of Dobesh et al. [18].

In the MechChem model, tension was obtained by solving Equation 2.9 numerically in Matlab (MathWorks, Natick, MA) with the ode23 solver.

\section{Results}

The experimental data of Dobesh and colleagues in skinned cardiac muscle (Table 2 and Figure $2 \mathrm{~A}$ of the original article) [18] is compared to the MechChem (Figure 2.4A) and the Hill (Figure 2.4B) model-generated $\left[\mathrm{Ca}^{2+}\right]$-tension relationships in Figure 2.4. In Figure 2.4A best-fit curves are shown, as obtained by the MechChem model after proper adjustment of the three parameters $K_{\text {TnCaO }}, C_{f}$ and $C_{s}$ per curve. Similarly, in Figure 2.4B, the curves are best-fit simulations, obtained by the Hill-type model, after proper adjustment of parameters $E C_{50}, n_{H}$ and $S_{\max }$. The lower panels, $4 C$ and $4 \mathrm{D}$, show the fitting errors per curve as a function of the $\left[\mathrm{Ca}^{2+}\right]$ for the MechChem and Hill-type model, respectively. The black lines indicate the median value of the error. For the 
MechChem model, there is no clear common pattern, and the error does not significantly differ from zero. For the Hill-type model, the error values show a clear common pattern, showing a consistent underestimation at the beginning of the upslope and the very last data points and a consistent overestimation at the location where the curves bend towards the maximum value. Thus, in contrast with the MechChem model, the shape of the tension curve, as simulated with the Hill-type model, is clearly different from the measured data. The MechChem curves are asymmetric with a relatively sharp upward bend for low $\left[\mathrm{Ca}^{2+}\right]$ and a moderate bending toward the saturation level.
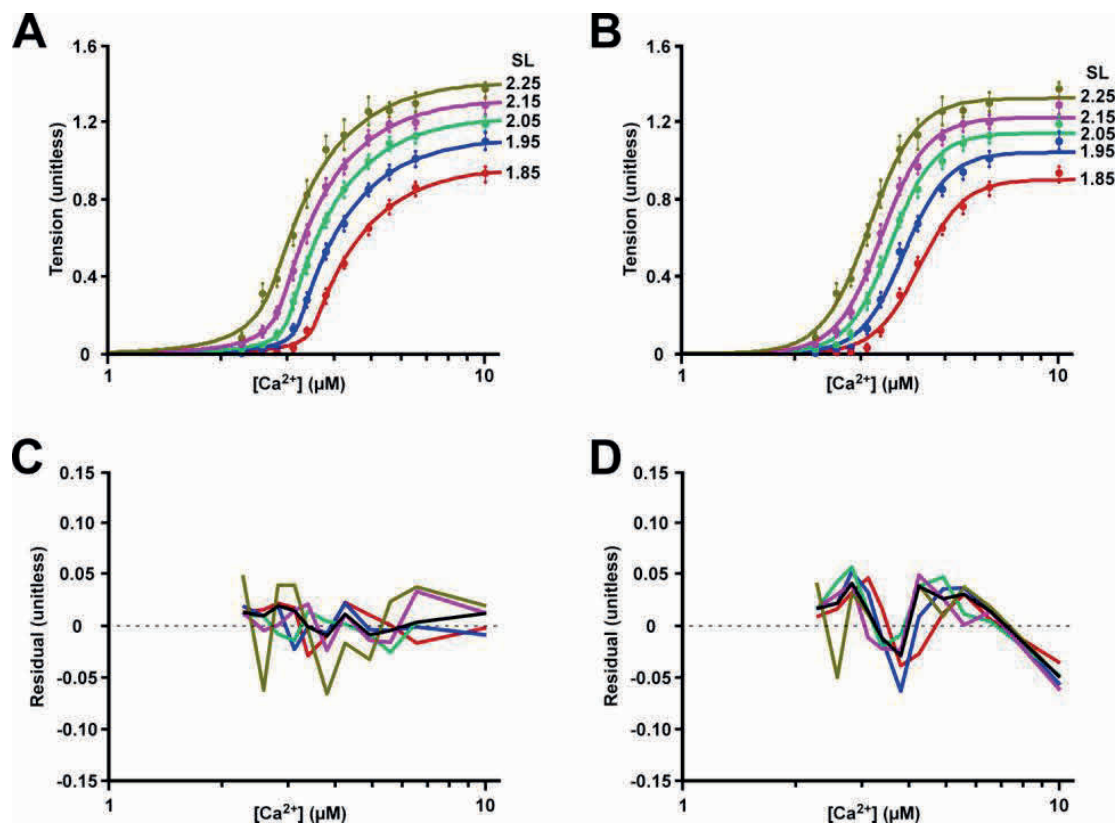

Figure 2.4: Static $\left[\mathrm{Ca}^{2+}\right]$-Tension Relationship in Skinned Muscle. (A). The MechChem model generated $\left[\mathrm{Ca}^{2+}\right]$-tension relationship with all parameters fitted per curve. The dots on the curve are plotted from the data presented in Table 2 and Figure 2A of Dobesh et al. 2002 [18]. (B) Hill model generated [ $\mathrm{Ca}^{2+}$ ]-tension curves. The experimental data points from Figure 2.4A were superimposed on the model generated curves. The error between the experimental data and both the (C) MechChem model generated $\left[\mathrm{Ca}^{2+}\right]$-tension curves and (D) the Hill model generated $\left[\mathrm{Ca}^{2+}\right]$-tension curves is shown. 
In Table 2.1, the triplets of parameter values for the simulated curves are shown per sarcomere length for both the MechChem model and the fits according to the Hill-type model. The best-fit values we obtained for the Hill-type model were similar to those obtained by Dobesh et al. [18]. The differences we report are likely a result of either a different fitting routine or slight errors when the data were extracted from a published figure. On average, the root mean square error RSME for the MechChem model is smaller than that for the Hill-type model. The parameter $C_{f}$ varied by a maximum of $6.5 \%$ at different $S L^{\prime} S$, whereas $K_{\text {Tncao }}$ and $C_{s}$ decreased by $43.2 \%$ and $70.6 \%$, respectively. Interestingly, for the MechChem model, parameter values $K_{\text {TnCaO }}$ and $C_{s}$ exhibit strong interdependency. Our analyses showed that for a change in $K_{\text {TnCaO }}$ by a factor of $a(a>1), C_{s}$ changed according to Equation 2.12 while exhibiting little influence on the resulting $\left[\mathrm{Ca}^{2+}\right]$-tension relationship.

$$
a * K_{\text {TnCa0 }} \approx a^{3} * C_{s}
$$

According to the abovementioned interdependency, it is possible that either $K_{\text {Tncao }}$ or $C_{s}$ changes with $S L$. We assume that $K_{T n C a O}$ is the parameter that changes with $S L$ because the necessary increase in $K_{T n C a O}$ is much less than that in $C_{s}$. Additionally, a large body of research has shown that the sensitivity of $\mathrm{Tn}$ to bind $\mathrm{Ca}^{2+}$ changes with $S L[18,29,30]$. Therefore, we performed a best fit by varying $K_{\text {TnCaO }}$ while keeping $C_{f}$ and $C_{s}$ fixed at their average values. The resulting fit is shown in Figure 2.5. As shown in Table 2.1, the fit is nearly as good as the fit by variation of all three parameters. For the Hill-type model, all parameters vary with sarcomere length, albeit that cooperativity only slightly diminishes with sarcomere length.

In the results, obtained with the MechChem model, cooperativity is apparent from the steep upslope of the $\left[\mathrm{Ca}^{2+}\right]$-tension relationship (Figure 2.4A), i.e. the sharp upward bend in the $\left[\mathrm{Ca}^{2+}\right]$-tension relationship that begins at $\left[\mathrm{Ca}^{2+}\right]$ of about $2.5 \mu \mathrm{M}$. With increasing $S L$, there is an increase in both maximum tension development and slope in both the model-generated and experimental $\left[\mathrm{Ca}^{2+}\right]$-tension relationships. Furthermore, the $\left[\mathrm{Ca}^{2+}\right]$ required for half maximum tension development $\left(E C_{50}\right)$ 
decreases with longer SL'S indicating the increasing sensitivity of tension development in the thin filament to $\mathrm{Ca}^{2+}$. The experiments of Dobesh et al. [18] showed a decrease in $E C_{50}$ from $4.28 \mu \mathrm{M}$ to $3.16 \mu \mathrm{M}$ with $S L$ increasing from $1.85 \mu \mathrm{m}$ to $2.25 \mu \mathrm{m}$ (as determined by the Hill fit in our analyses) (Figure 2.4B, Table 2.1). In the MechChem simulations covering the same SL range, $E C_{50}$ decreased from $4.18 \mu \mathrm{M}$ to $3.17 \mu \mathrm{M}$ when fitting the model to the $\left[\mathrm{Ca}^{2+}\right]$-tension curves per SL (Figure 2.4A).

Table 2.1: Parameter values of fit model to experimental data

\begin{tabular}{|c|c|c|c|c|c|c|}
\hline Sarcomere length & $\mu \mathrm{m}$ & 1.85 & 1.95 & 2.05 & 2.15 & 2.25 \\
\hline \multicolumn{7}{|l|}{ MechChem $n=3$} \\
\hline$K_{\text {TnCaO }}$ & $\mu \mathrm{M}$ & 10.09 & 9.46 & 8.63 & 7.14 & 5.73 \\
\hline$C_{f}$ & $10^{6} \cdot \mathrm{m}^{-1} \mathrm{~S}$ & 1.85 & 1.95 & 1.97 & 1.96 & 1.95 \\
\hline$C_{s}$ & $\left.\mathrm{~S}^{-1} 1\right)$ & 9.27 & 8.66 & 7.32 & 4.61 & 2.73 \\
\hline RMSE & $\mathrm{S}$ & 0.017 & 0.013 & 0.012 & 0.017 & 0.040 \\
\hline \multicolumn{7}{|c|}{ MechChem $\mathrm{n}=3$ with fixed: $C_{f}=1.93 \cdot 10^{6} \mathrm{~m}^{-1} \mathrm{~S}, C_{s}=6.5 \mathrm{~S}^{-1}$} \\
\hline$K_{T n C a O}$ & $\mu \mathrm{M}$ & 9.26 & 8.56 & 8.18 & 7.91 & 7.48 \\
\hline RMSE & $\mathrm{S}$ & 0.024 & 0.012 & 0.019 & 0.021 & 0.053 \\
\hline \multicolumn{7}{|l|}{ Hill-type } \\
\hline $\mathrm{Ca}_{50}$ & $\mu \mathrm{M}$ & 4.28 & 3.9 & 3.6 & 3.4 & 3.16 \\
\hline$n_{H}$ & - & 7.3 & 7.5 & 7.5 & 7.2 & 6.9 \\
\hline$S_{\operatorname{Max}}$ & $\mathrm{S}$ & 0.90 & 1.04 & 1.14 & 1.22 & 1.32 \\
\hline RMSE & $\mathrm{S}$ & 0.028 & 0.037 & 0.033 & 0.032 & 0.033 \\
\hline
\end{tabular}

Figure 2.6A and 2.6B show the MechChem simulation of tension and degree of activation along the thin filament in the single overlap zone, respectively, at different $\left[\mathrm{Ca}^{2+}\right]^{\prime} \mathrm{s}(3,4,5 \mu \mathrm{M}$ with $\mathrm{SL}=2.05 \mu \mathrm{M})$. These values were chosen to show the results in the middle range of the experimental conditions. The MechChem model predicts that the density of bound $X B^{\prime}$ s increases with the position $x$ along the thin filament. 
The plateaus shown in Figure 2.6B represent the full activation of $T n^{\prime}$ s, i.e. all binding sites on that section of the thin filament are exposed for $X B$ binding.

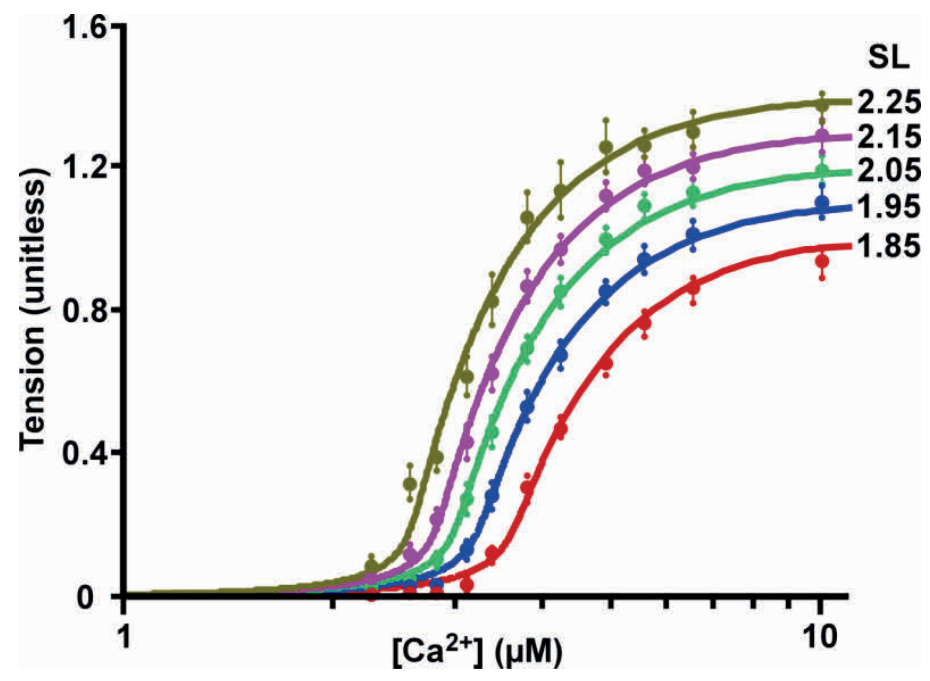

Figure 2.5: MechChem generated $\left[\mathrm{Ca}^{2+}\right]$-Tension Relationship in Skinned Muscle. The MechChem model generated $\left[\mathrm{Ca}^{2+}\right]$-tension relationship with $K_{\text {Tncao }}$ changing with $S L$, while setting $\mathrm{Cf}=1.93 \cdot 10^{6} \mathrm{~m}^{-1} \mathrm{~S}$ and $C s=6.5 \mathrm{~S}^{-1}$. The unit $\mathrm{S}$ refers to the unit of tension as shown by Dobesh et al. [18]. The dots on the curve are plotted from the data presented in Table 2 and Figure 2A of Dobesh et al. 2002 [18].

A

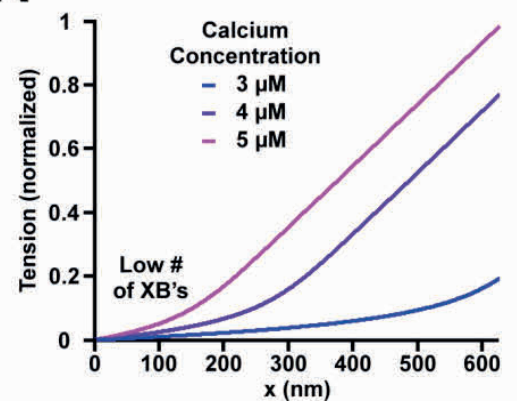

B

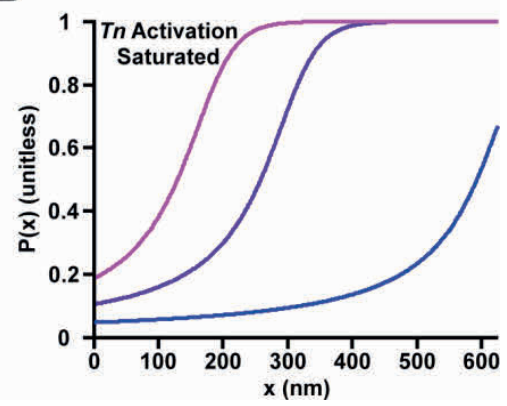

Figure 2.6: Tension and the proportion of activated troponin complexes $(T n) P(x)$ in the thin filament along the single overlap region. A sarcomere with length $2.05 \mu \mathrm{m}$ was modeled with constant calcium concentrations ([Ca]). The blue, purple, and magenta lines represent a $[\mathrm{Ca}]$ of 3,4 , and $5 \mu \mathrm{M}$, respectively. (A) The plot shows the non-linear cumulative tension developed at position $x$ along the single overlap region. (B) $P(x)$ is displayed. The plateaus reached at [Ca]'s of 4 and $5 \mu \mathrm{M}$ represent the full activation of $T n^{\prime}$ s, i.e. all binding sites on that section of the thin filament are exposed for $X B$ binding. 


\section{Discussion}

The methods presented here provide a novel view of a mechanochemical mechanism of cooperativity in cardiac sarcomeres. The intrinsic cooperativity of $\mathrm{Ca}^{2+}$ binding to an in-register pair of $T n^{\prime}$ s, i.e., cooperativity in the absence of mechanical tension, is characterized by a base cooperativity coefficient $n=3$ according to experimental data reported by Sun et al. [20]. In skinned muscle experiments of Dobesh et al. [18], cooperativity is considerably higher, as characterized by a Hill coefficient of 7 . We propose that the latter boost occurs by a mechanism where mechanical tension in the thin filament strengthens the chemical binding of $\mathrm{Ca}^{2+}$ to $\mathrm{Tn}$. With each $\mathrm{XB}$ bound, the tension in the thin filament increases toward the Z-disk. This tension increments the energy required to deactivate a $\mathrm{Tn}$, thus increasing the affinity of $\mathrm{Ca}^{2+}$ for $\mathrm{Tn}$ towards the Z-disk. The model-simulated $\left[\mathrm{Ca}^{2+}\right]$-tension relation appeared in good agreement (Table 2.1) with the experimental data from Dobesh et al. [18], thus supporting the hypothesis that an important contribution to cooperativity in cardiac muscle is delivered by the mechanical tension in the thin filament acting over a long range, i.e. the entirety of the thin filament.

\section{Comparing measured $\left[\mathrm{Ca}^{2+}\right]$-tension relation with MechChem model simulations}

Considering the MechChem simulation with parameters $C_{f}$ and $C_{s}$ fixed and $K_{\text {TnCaO }}$ depending on SL (Figure 2.5, Table 2.1), the model-generated $\left[\mathrm{Ca}^{2+}\right]$-tension curves resembled the experimental data of Dobesh et al. [18]. Typically, the $\log \left[\mathrm{Ca}^{2+}\right]$ - tension curve is S-shaped from zero tension at low $\left[\mathrm{Ca}^{2+}\right]$ to a saturated value at high $\left[\mathrm{Ca}^{2+}\right]$. The steepness of the slopes in the experimental data of Dobesh et al. [18] indicates cooperativity with a Hill coefficient around 7. At low $\left[\mathrm{Ca}^{2+}\right]$, the $\left[\mathrm{Ca}^{2+}\right]$ - tension bends up sharply, giving rise to the steepest part of the curve, indicating a high $\left[\mathrm{Ca}^{2+}\right]$ sensitivity. With maximum [ $\left.\mathrm{Ca}^{2+}\right](10 \mu \mathrm{M})$ and SL increasing from $1.85 \mu \mathrm{m}$ to $2.25 \mu \mathrm{m}$, the simulated peak tension increased by $49 \%$ and the measured value by $47 \%$. Simulations also produced an increase in $\mathrm{Ca}^{2+}$ sensitivity, shown by a decrease of $K_{\text {CaTno }}$ from 16.6 down to $11.8 \mu \mathrm{M}$, implying a leftward shift of the $\left[\mathrm{Ca}^{2+}\right]$-tension curves with increasing SL. The shape of the MechChem curves (Figure 2.4A, 2.5) covers the 
experimental data better than the sigmoid Hill-curves (Figure 2.4B), as shown by the residuals in Figure $2.4 \mathrm{C}$ and $2.4 \mathrm{D}$, especially for the mid and high $\left[\mathrm{Ca}^{2+}\right]$. It should be noted that for low $\left[\mathrm{Ca}^{2+}\right]$ the residuals of the MechChem curves are somewhat larger, probably because of the steep gradient of that part of the curve. The discrepancy between the results of the MechChem model at low $\left[\mathrm{Ca}^{2+}\right]$ and the experimental results may be further explained by assuming some dispersion in sarcomere length and in the length of the thin and thick filaments, causing the sharp uprising bend to be smeared out, thus moderating the slope in that part of the curve.

\section{Proposed mechanism of cooperativity in the MechChem model}

Cooperativity is assumed to have two components. The first is the intrinsic cooperativity related to binding of $\mathrm{Ca}^{2+}$ to $\mathrm{Tn}$ in absence of mechanical tension. The related intrinsic cooperativity coefficient is set to 3 according to the findings by Sun et al. [20]. Secondly, the mechanochemical component boosts the cooperativity from the intrinsic coefficient of 3 up to the experimentally measured value of about 7 .

Sun et al. [20] inhibited tension development in trabeculae with blebbistatin and found that in the absence of tension the Ca-dependent activation of $T n^{\prime}$ s along the thin filament remained cooperative with a Hill coefficient $n_{H}=3$. The Hill coefficient indicates the minimum number of binding sites that cooperate. Each $T n$ contains 3 binding sites for $\mathrm{Ca}^{2+}$ [20], but they do not all have the same affinity and are not likely to fully cooperate. Galińska-Rakoczy et al. [17] showed that the C-terminal section of $T n l$ touches the $T m$ on the adjacent actin strand, thus coupling both $T n^{\prime}$ s, forming an in-register pair $T n_{2}$. Consequently, for each $T n_{2}$, there are 6 binding sites available for inherent cooperation. Apparently, cooperativity is partial, resulting in $n \approx 3$.

By introduction of the MechChem model of tension-driven cooperativity, we propose that mechanical tension in the thin filament results in the strengthening of the binding between $\mathrm{Ca}^{2+}$ and $\mathrm{Tn}$, thereby hindering deactivation, implying that tension lowers the energy associated with $\mathrm{Ca}^{2+}$ being bound to $\mathrm{Tn}$. Combining intrinsic cooperativity with the mechanochemical mechanism results in the MechChem model, which results 
display a striking resemblance to the experimentally measured tension as a function of $\left[\mathrm{Ca}^{2+}\right]$ in skinned muscle preparations (Figure 2.4, 2.5). Many correlations between change of $\mathrm{Ca}^{2+}$ binding properties and mechanical events have been reported.

Rieck et al. [31] showed that even in the absence of tension in the thin filament, the formation of strong $X B^{\prime}$ s stabilized the open conformation of the Tn. Since $X B^{\prime}$ s can only form if a nearby $T n$ is in the unblocked state, that $T n$ may help to unblock the inregister $T n$, thus enhancing the formation of additional $X B^{\prime} s$ attached to the paired actin strand. Furthermore, Isambert et al. [32] showed that the rigidity of the thin filament decreased when Tm was in the unblocked state as compared to being in the blocked state. This finding shows that there is a mechanical coupling between the conformational changes of $T n$ and elastic properties of the thin filament. More recently, Desai et al. [33] directly showed that myosin binding was necessary for complete activation of the thin filament upon partial activation due to calcium binding.

The MechChem simulated best-fit $\left[\mathrm{Ca}^{2+}\right]$-tension curve was determined for intrinsic cooperativity coefficients $n=1,2,3$ and shown in the supplementary material (2.S1 Figure). The fit is excellent when $n=3$, while for $n=1$ or 2 , the curves are not as steep as in the physiological situation. In the experiments by Sun et al., development of mechanical tension was shown not to have an effect on cooperativity, i.e. the Hill coefficient remained as low as 3. As they mentioned in their article [20], they could not exclude the possibility that the applied fluorescent probes attached to the various structures of the Tn complex may change the properties of $T n$ to some degree. We think that these probes may inhibit the mechanochemical enhancement of $\mathrm{Ca}^{2+}$ affinity by tension.

\section{Cooperativity in the MechChem model vs. the Hill model}

The $\left[\mathrm{Ca}^{2+}\right]$-tension relation in skinned muscle has generally been characterized by a modified Hill curve that takes on a symmetric s-shape [34]. The $\left[\mathrm{Ca}^{2+}\right]$-tension relation simulated by our model is asymmetric (Figure 2.4A, 2.4C); the initial steep rise in tension generated with additional $\mathrm{Ca}^{2+}$ decreases closer to saturation. Whereas 
Dobesh et al. fitted symmetric curves, they admitted that the Hill curves consistently overestimated the tension developed in the sarcomeres as the curves rounded toward saturation (Figure 2.4B, 2.4D). In an attempt to account for the asymmetry, Dobesh et al. [18] proposed that the experimental data be fit to 2 Hill coefficients that meet at $E C_{50}$ for each curve. In the Mech Chem model, the $\left[\mathrm{Ca}^{2+}\right]$-tension relation is already asymmetric, so the introduction of an additional parameter is not necessary to reshape the curve.

Both the Hill-type model and the MechChem model require 3 parameters per curve. By fitting the models to the experimental data for each sarcomere length separately, in the Hill-type model all 3 parameters appeared to depend on SL, albeit that dependency of the Hill coefficient $n_{H}$ appeared weak (Table 2.1). In the MechChem model, parameters $C_{f}$ and $C_{s}$ did not depend clearly on SL, thus hinting us to keep these parameter values fixed, while only reestimating the equilibrium constant $K_{\text {CaTno, }}$ expressing $\mathrm{Ca}^{2+}$ affinity in absence of mechanical tension. The resulting fits were nearly as good (Figure 2.5), suggesting that $\mathrm{Ca}^{2+}$ affinity apparently depends somehow on SL, while the other parameters were general to all SL's.

Our model comprises two mechanisms of cooperativity that generate the $\left[\mathrm{Ca}^{2+}\right]$ tension relationship, i.e. tension in $\mathrm{Tm}$ strengthening the bond between $\mathrm{Ca}^{2+}$ and $\mathrm{Tn}$ and intrinsic cooperative activation of in-register pairs of $T n$. The Hill function is based on common chemical equilibrium and provides the basis for many current models of $\mathrm{Ca}^{2+}-\mathrm{Tn}$ binding. For example, Rice et al. [22] utilize a modified Hill function to model the transition between the active and inactive states of $T n$. The peak intracellular $\left[\mathrm{Ca}^{2+}\right]$ reaches $1.45 \mu \mathrm{M}$ in the Rice model, a concentration that the Hill function mimics well. The Hill model does not provide a physical explanation for the mechanism of cooperativity in cardiac muscle, but instead utilizes a coefficient that characterizes the steepness of the $\left[\mathrm{Ca}^{2+}\right]$-tension relation. Additionally, the Hill model was initially developed to understand the cooperative binding of oxygen to hemoglobin [34], where it makes physical sense that a first chemical binding of oxygen on a hemoglobin molecule will facilitate subsequent bindings because oxygen-binding sites on 
hemoglobin are separated by only 2.5 to $3.5 \mathrm{~nm}$ [35]. To reach the high physiologic level of cooperativity, several $T n^{\prime}$ 's must interact. Because $T n^{\prime}$ 's are separated by about $35 \mathrm{~nm}$ along a single actin strand within the thin filament, it is physically difficult to explain that a chemical binding can influence the binding of a different molecule that far away by conventional chemical principles.

It has been shown that the sensitivity of $T n$ to bind $\mathrm{Ca}^{2+}$ changes with $S L[18,29]$, a characteristic implemented in the MechChem model. There is currently no clear consensus regarding the mechanism behind length dependent $\mathrm{Ca}^{2+}$ sensitivity in cardiac muscle (for review, [36]). One proposed mechanism is that the lattice spacing decreases with longer SL, moving myosin heads closer to the thin filament and rendering the thin filament more sensitive to $\mathrm{Ca}^{2+}$ by enhancing binding [37]. However, the lattice spacing hypothesis has been questioned after it has been shown that muscle length does not necessarily correlate with myofilament spacing [30]. It has also been proposed that phosphorylation of sites on $\mathrm{Tn} /$ by protein kinase $\mathrm{A}$ and protein kinase $\mathrm{C}$ alters the $\mathrm{Ca}^{2+}$ sensitivity and could prove significant in the regulation of length dependent activation [14]. Conversely, Lee et al. have shown that the increase of passive tension in titin leads to increased $\mathrm{Ca}^{2+}$ sensitivity [38]. It is possible that the change in $K_{T n C a O}$ in the MechChem model is due to a combination of the abovementioned mechanisms.

\section{The MechChem model vs. nearest neighbor cooperativity models}

As already indicated in the introduction, various models have been developed on the basis of nearest neighbor cooperativity, based on $R U-R U, X B-X B$ or $X B-R U$ interactions. These models generally result in Hill-type $\left[\mathrm{Ca}^{2+}\right]$-tension relations that fit accurately to experimental data. However, the curves are slightly, but systematically different from the MechChem curves (Figure 2.4). It is not clear yet if these differences are sufficiently strong to make a choice between the two model types. We predict a clear difference to be expected, yet we do not currently have the means to test the model. In a tension bearing sarcomere, the MechChem model predicts that the hindrance to Tn deactivation imposed by high tension in the thin filament causes a higher 
concentration of activated $\mathrm{Tn}^{\prime}$ s, bound $\mathrm{Ca}^{2+}$, and $\mathrm{XB}^{\prime}$ s toward the Z-disk end of the single overlap region where tension is highest (Figure 2.5B). With the nearest neighbor hypothesis, no preference is to be expected on the location of activated $T n^{\prime}$ s. However, although not explicitly noted, the MechChem model does take into account some of the nearest neighbor cooperativity mechanisms. The development of tension in the thin filament begets more tension development. Thus, $X B^{\prime}$ s recruit more $X B^{\prime}$ s. Additionally, the tension in the thin filament at position $x$ strongly determines whether the $T n$ is active at point $x$ (XB-RU cooperativity). $R U-R U$ cooperativity is accounted for in the MechChem model through the intrinsic cooperativity coefficient $n$.

\section{Comparison with long range cooperativity hypotheses}

Most of the models previously discussed have included only local cooperativity mechanisms, yet there are also models and hypotheses that incorporate cooperative mechanisms acting along the entirety of the thin filament. Brandt and colleagues [39] proposed that the mutual overlap of $\mathrm{Tm}$ molecules under $\mathrm{Tn}$ causes a simultaneous unblocking or blocking of all Tm molecules along the filament. Conversely, the "cooperative realignment of binding sites", a model developed by Daniel and colleagues [26], ignores the effects of $\mathrm{Tm}$ but looks instead at the possible impact of strain on the thin filament. Hence, this model can predict cooperativity in tension development but not in activation. Still others attribute the cooperative effect to the constant volume property of the myofiber matrix. The stretch of sarcomeres causes the thick and thin filaments to squeeze closer together increasing the probability of $X B$ binding [24]. Like the cooperative realignment of binding sites, the lattice spacing hypothesis can account for cooperative tension generation but not activation.

The model designed by Land \& Niederer [40] represented the entire thin filament, a compilation of $26 R U^{\prime}$ s. They hypothesized that the state (position) of the Tm molecule in each $R U$ has a corresponding free energy determined by the state of neighboring $X B^{\prime} S$ and $R U^{\prime}$ s. The energy term is used to compute the probability of a $R U$ being blocked or unblocked. While the simulated results are consistent with available experimental data and insight can be gained through the Land \& Niederer model, it is 
composed of a system of 750 ordinary differential equations. The tension-driven cooperativity model we present consists of a single ordinary differential equation and three key parameters, so we present a highly simplified model that still captures behaviors shown in available experimental data. Our model, like the Land \& Niederer [40] model, includes an energy term in the computations of state $P$. It is a mechanical energy term related to the tension within the thin filament that increases along the thin filament from the start of the single overlap region towards the Z-disk. Our model is different from others because the mechanics of the thin filament $(S(x))$ directly impact the related chemistry $\left(\mathrm{Ca}^{2+}\right.$ binding to $\left.\mathrm{Tn}\right)$ whereas most of the other models view the mechanics and chemistry separately.

Izakov et al. have hypothesized that the number of strongly bound $X B^{\prime}$ s along the thin filament affects the binding affinity of $\mathrm{Ca}^{2+}$ to $\mathrm{Tn}$ and have implemented this idea in a computational model [41]. Landesberg and Sideman proposed a similar model, but it was a loosely coupled model meaning that $\mathrm{Ca}^{2+}$ was not required to remain bound for the $T n$ to remain active, but bound $X B^{\prime}$ s were adequate to do so [42]. Our model differs because we propose that the tension in the thin filament contributes to an increment energy required to unbind $\mathrm{Ca}^{2+}$ from $\mathrm{Tn}$. This energy increases along the thin filament toward the Z-disk as tension increases. In the models discussed above, the global affinity for $\mathrm{Ca}^{2+}$ to bind to $\mathrm{Tn}$ will change based on the number of strong $X B^{\prime}$ s. Within our model, however, the affinities increase with tension in the thin filament, being explained by a linear increase of binding energy with tension in the thin filament.

\section{Mechanism of relaxation as calcium concentration decreases}

Due to the interaction between mechanics and chemistry within the model, neither mechanics nor chemistry fully account for the activation or subsequent deactivation of the thin filament. While we propose that high tension in the thin filament hinders deactivation, a decrease in intracellular $\left[\mathrm{Ca}^{2+}\right]$ will trigger relaxation. Due to the buildup of tension within the thin filament as shown in Figure 2.3, there is always a loose end closer to the mid-line in which little tension is developed. The hindrance to 
deactivation imposed by high tension does not exist in these areas, thus promoting the deactivation of the $T n^{\prime}$ s closest to the mid-line first. We propose that the areas of highest tension (closest to the z-disk) are the latest to deactivate.

\section{Heterogeneity of cross-bridge density}

Our model has provided a potentially experimentally testable hypothesis. Model results suggest directionality in the dispersion of $X B^{\prime}$ s along the thin filament with a higher concentration of $X B^{\prime}$ s closer to the Z-disk. Desai and colleagues [33] were recently able to fluorescently label single myosin heads and observe single bindings to the thin filament. They observed that although myosin binding activated the $R U$, there was no directionality in the binding of $X B^{\prime}$ s. However, tension was not developed in this model because the myosin heads were not tethered to the thick filament. Additional experimental evidence is needed to test the hypothesis of $X B$ dispersion in the loaded thin filament. The difficulty lies in developing an experimental approach that enables viewing of each individual $X B$ binding in a skinned muscle under tension.

\section{Model Limitations}

The model presented here assumes that when an area on the thin filament becomes unblocked, $X B^{\prime}$ s are automatically formed. Thus, the fraction of unblocking is proportional to the $X B$ - force developed along the strand. The assumption that $X B^{\prime} S$ form automatically when $R U^{\prime}$ s are activated is not physiologically accurate. We expect this to be corrected upon the explicit incorporation of the different steps of the $X B$ cycle. However, the experimentally measured tension in skinned muscle preparations is obtained after the steady state has been reached. Hence, the experimental data is time-independent.

The current model results are limited to the $\left[\mathrm{Ca}^{2+}\right]-$ tension relationship. The introduction of dynamics such as the $X B$ cycle requires additional assumptions to be made and additional unknown parameters added. However, the next logical step for the model is the implementation of the $X B$ cycle that will be studied in the isometric twitch. The MechChem model is a mean field approximation, so it is highly simplified. 
Specific spatial details such as individual binding sites are not accounted for, but the simplification reduces computational cost considerably.

\section{Conclusions}

A novel mechanochemical model of tension generation by the sarcomere has been developed based on long range cooperativity imposed by mechanical tension in the thin filament and intrinsic cooperativity resulting from the interaction between the calcium-binding sites on the in-register troponin complexes. Simulated $\left[\mathrm{Ca}^{2+}\right]$-tension curves resembled those obtained in steady state isometric muscle experiments. Thus, our results support the hypothesis that high tension in the thin filament impedes deactivation by increasing the energy required to detach calcium from the troponin complex. Furthermore, we found that the tension in the thin filament was relatively low toward the beginning of the single overlap region close to the mid-line of the sarcomere but increased steeply in the overlap region closer to the Z-disk. Model simulations suggest that the concentration of calcium bindings to the troponin complexes and active $X B^{\prime}$ s are low at the free end of the thin filament and saturated closer to the Z-disk. Future experimental studies are needed to test the latter property, indicating the validity of our hypothesis on the cooperative effect of tension in the thin filament on force generation by the cardiac sarcomere. 


\section{S1 Appendix}

\section{Overlap length}

Single overlap length $x_{\max }$ of the thin and thick filaments depends on SL and length of the thin filament $I_{\text {thin }}$, the myosin filament $I_{\text {thick }}$, and the bare zone $I_{\text {bare }}$ in the middle of the thick filament according to the formulations of Rice et al (22):

$$
\begin{gathered}
x_{\max }=x_{z}-x_{c} \\
x_{z}=\min \left(\frac{l_{\text {thick }}}{2}, \frac{l_{\text {sarc }}}{2}\right) \\
x_{c}=\max \left(\frac{l_{\text {sarc }}}{2}-\left(l_{\text {sarc }}-l_{\text {thin }}\right), \frac{l_{\text {bare }}}{2}\right)
\end{gathered}
$$

The values of the independent length components utilized in the model are as follows: $I_{\text {thin }}=1.2 \mu \mathrm{m}, I_{\text {bare }}=0.1 \mu \mathrm{m}, I_{\text {thick }}=1.6 \mu \mathrm{m}$ (9).

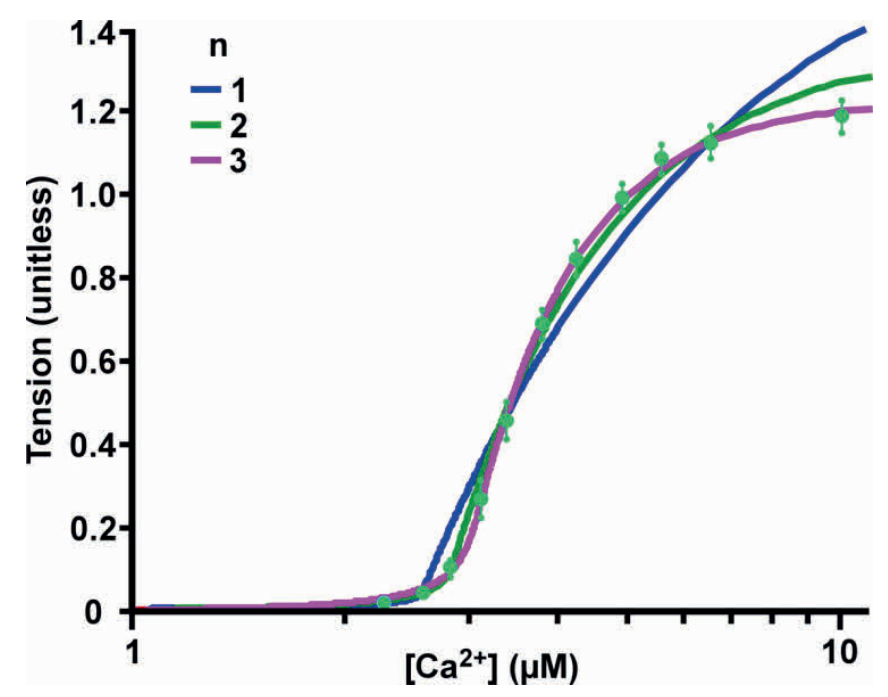

2.S1 Figure: $\left[\mathrm{Ca}^{2+}\right]$-Tension relationship alterations in intrinsic cooperativity. The cooperativity constant $n$ is altered between values of 1 (blue), 2 (green), and 3 (magenta) at $S L=2.05 \mu \mathrm{m}$. The green dots on the plot are the experimental data of Dobesh et al, and the best fit of the model to the experimental data of Dobesh et al. was found for each $n[1,2,3] . C_{s}=[134.90,24.07,7.32]\left(S^{-1}\right) C_{f}=[0.00293,0.00220,0.00197]\left(\mathrm{nm}^{-1} \mathrm{~S}\right)$ $K_{\text {TnCaO }}=[618.97,22.14,8.63](\mu \mathrm{M})$. 


\section{References}

1. Ringer S. A further Contribution regarding the influence of the different Constituents of the Blood on the Contraction of the Heart. The Journal of Physiology. 1883;4(1):29-42. doi: 10.1113/jphysiol.1883.sp000120.

2. Allen DG, Kentish JC. The cellular basis of the length-tension relation in cardiac muscle. Journal of Molecular and Cellular Cardiology. 1985;17(9):821-40. doi: http://dx.doi.org/10.1016/S0022-2828(85)800973.

3. Campbell K. Impact of myocyte strain on cardiac myofilament activation. Pflugers Arch - Eur J Physiol. 2011;462(1):3-14. doi: 10.1007/s00424-011-0952-3.

4. Gordon AM, Homsher E, Regnier M. Regulation of Contraction in Striated Muscle2000 2000-0104 00:00:00. 853-924 p.

5. Huxley HE. Electron microscope studies of the organisation of the filaments in striated muscle. Biochimica et Biophysica Acta. 1953;12(1-2):387-94. doi: http://dx.doi.org/10.1016/0006-3002(53)90156-5.

6. Huxley HE. The Double Array of Filaments in Cross-Striated Muscle. The Journal of Biophysical and Biochemical Cytology. 1957;3(5):631-48. PubMed PMID: PMC2224118.

7. Hanson J, Lowy J. The structure of F-actin and of actin filaments isolated from muscle. Journal of Molecular Biology. 1963;6(1):46-IN5. doi: http://dx.doi.org/10.1016/S0022-2836(63)80081-9.

8. Levine R, Weisberg A, Kulikovskaya I, McClellan G, Winegrad S. Multiple Structures of Thick Filaments in Resting Cardiac Muscle and Their Influence on Cross-Bridge Interactions. Biophysical Journal. 2001;81(2):1070-82. doi: http://dx.doi.org/10.1016/S0006-3495(01)75764-5.

9. Poole DC, Lieber RL, Mathieu-Costello O. Myosin and actin filament lengths in diaphragms from emphysematous hamsters. Journal of applied physiology (Bethesda, Md : 1985). 1994;76(3):1220-5. Epub 1994/03/01. PubMed PMID: 8005866.

10. Gordon AM, Huxley AF, Julian FJ. The variation in isometric tension with sarcomere length in vertebrate muscle fibres. The Journal of Physiology. 1966;184(1):170-92. PubMed PMID: PMC1357553.

11. Potter JD. The content of troponin, tropomyosin, actin, and myosin in rabbit skeletal muscle myofibrils. Archives of Biochemistry and Biophysics. 1974;162(2):436-41. doi: http://dx.doi.org/10.1016/0003-9861(74)90202-1.

12. Solaro J. Chapter 30 - Mechanisms Regulating Cardiac Myofilament Response to Calcium. In: Cohen NSKTV, editor. Heart Physiology and Pathophysiology (Fourth Edition). San Diego: Academic Press; 2001. p. 519-26.

13. Yang S, Barbu-Tudoran L, Orzechowski M, Craig R, Trinick J, White H, et al. Three-Dimensional Organization of Troponin on Cardiac Muscle Thin Filaments in the Relaxed State. Biophysical Journal. 2014;106(4):855-64. doi: http://dx.doi.org/10.1016/j.bpj.2014.01.007.

14. Solaro RJ, Rarick HM. Troponin and Tropomyosin: Proteins That Switch on and Tune in the Activity of Cardiac Myofilaments. Circulation Research. 1998;83(5):471-80. doi: 10.1161/01.res.83.5.471.

15. McKillop DF, Geeves MA. Regulation of the interaction between actin and myosin subfragment 1 : evidence for three states of the thin filament. Biophysical Journal. 1993;65(2):693-701. PubMed PMID: PMC1225772.

16. Parmacek MS, Solaro RJ. Biology of the troponin complex in cardiac myocytes. Progress in Cardiovascular Diseases. 2004;47(3):159-76. doi: http://dx.doi.org/10.1016/j.pcad.2004.07.003.

17. Galińska-Rakoczy A, Engel P, Xu C, Jung H, Craig R, Tobacman LS, et al. Structural Basis for the Regulation of Muscle Contraction by Troponin and Tropomyosin. Journal of Molecular Biology. 2008;379(5):929-35. doi: http://dx.doi.org/10.1016/j.jmb.2008.04.062.

18. Dobesh DP, Konhilas JP, de Tombe PP. Cooperative activation in cardiac muscle: impact of sarcomere length. American Journal of Physiology - Heart and Circulatory Physiology. 2002;282(3):H1055H62. doi: 10.1152/ajpheart.00667.2001.

19. Holroyde MJ, Robertson SP, Johnson JD, Solaro RJ, Potter JD. The calcium and magnesium binding sites on cardiac troponin and their role in the regulation of myofibrillar adenosine triphosphatase. Journal of Biological Chemistry. 1980;255(24):11688-93.

20. Sun Y-B, Lou F, Irving M. Calcium- and myosin-dependent changes in troponin structure during activation of heart muscle. The Journal of Physiology. 2009;587(Pt 1):155-63. doi: 10.1113/jphysiol.2008.164707. PubMed PMID: PMC2670030. 
21. Razumova MV, Bukatina AE, Campbell KB. Different Myofilament Nearest-Neighbor Interactions Have Distinctive Effects on Contractile Behavior. Biophysical Journal. 2000;78(6):3120-37. doi: http://dx.doi.org/10.1016/S0006-3495(00)76849-4.

22. Rice JJ, Wang F, Bers DM, de Tombe PP. Approximate Model of Cooperative Activation and Crossbridge Cycling in Cardiac Muscle Using Ordinary Differential Equations. Biophysical Journal. 2008;95(5):2368-90. doi: http://dx.doi.org/10.1529/biophysj.107.119487.

23. Campbell SG, Lionetti FV, Campbell KS, McCulloch AD. Coupling of Adjacent Tropomyosins Enhances Cross-Bridge-Mediated Cooperative Activation in a Markov Model of the Cardiac Thin Filament. Biophysical Journal. 2010;98(10):2254-64. doi: http://dx.doi.org/10.1016/i.bpj.2010.02.010.

24. Tanner BCW, Farman GP, Irving TC, Maughan D W, Palmer BM, Miller M S. Thick-to-Thin Filament Surface Distance Modulates Cross-Bridge Kinetics in Drosophila Flight Muscle. Biophysical Journal. 2012;103(6):1275-84. doi: 10.1016/j.bpj.2012.08.014. PubMed PMID: PMC3447602.

25. Williams CD, Salcedo MK, Irving TC, Regnier M, Daniel TL. The length-tension curve in muscle depends on lattice spacing. Proceedings of the Royal Society B: Biological Sciences. 2013;280(1766):20130697. doi: 10.1098/rspb.2013.0697. PubMed PMID: PMC3730583.

26. Daniel TL, Trimble AC, Bryant Chase P. Compliant Realignment of Binding Sites in Muscle: Transient Behavior and Mechanical Tuning. Biophysical Journal. 1998;74(4):1611-21. doi: http://dx.doi.org/10.1016/S0006-3495(98)77875-0.

27. Moore JR, Campbell SG, Lehman W. Structural determinants of muscle thin filament cooperativity. Archives of Biochemistry and Biophysics. 2016;594:8-17. doi: http://dx.doi.org/10.1016/j.abb.2016.02.016.

28. Bers DM. Calcium Cycling and Signaling in Cardiac Myocytes. Annual Review of Physiology. 2008;70(1):23-49. doi: doi:10.1146/annurev.physiol.70.113006.100455. PubMed PMID: 17988210.

29. Kentish JC, ter Keurs HE, Ricciardi L, Bucx JJ, Noble MI. Comparison between the sarcomere length-force relations of intact and skinned trabeculae from rat right ventricle. Influence of calcium concentrations on these relations. Circulation Research. 1986;58(6):755-68. doi: 10.1161/01.res.58.6.755.

30. de Tombe PP, Mateja RD, Kittipong T, Mou YA, Farman GP, Irving TC. Myofilament Length Dependent Activation. Journal of molecular and cellular cardiology. 2010;48(5):851-8. doi: 10.1016/j.yjmcc.2009.12.017. PubMed PMID: PMC2854194.

31. Rieck DC, Li K-L, Ouyang Y, Solaro RJ, Dong W-J. Structural basis for the in situ Ca2+ sensitization of cardiac troponin $\mathrm{C}$ by positive feedback from force-generating myosin cross-bridges. Archives of Biochemistry and Biophysics. 2013;537(2):198-209. doi: http://dx.doi.org/10.1016/j.abb.2013.07.013.

32. Isambert H, Venier P, Maggs A, Fattoum A, Kassab R, Pantaloni D, et al. Flexibility of actin filaments derived from thermal fluctuations. Effect of bound nucleotide, phalloidin, and muscle regulatory proteins. Journal of Biological Chemistry. 1995;270(19):11437-44. doi: 10.1074/jbc.270.19.11437.

33. Desai RA, Geeves MA, Kad NM. Using Fluorescent Myosin to Directly Visualize Cooperative Activation of Thin Filaments. Journal of Biological Chemistry. 2014. doi: 10.1074/jbc.M114.609743.

34. Hill AV. The Combinations of Haemoglobin with Oxygen and with Carbon Monoxide. I. Biochemical Journal. 1913;7(5):471-80. PubMed PMID: PMC1276492.

35. McConnell HM. Spin-Label Studies of Cooperative Oxygen Binding to Hemoglobin. Annual Review of Biochemistry. 1971;40(1):227-36. doi: doi:10.1146/annurev.bi.40.070171.001303. PubMed PMID: 4330577.

36. Kobirumaki-Shimozawa F, Inoue T, Shintani SA, Oyama K, Terui T, Minamisawa S, et al. Cardiac thin filament regulation and the Frank-Starling mechanism. The Journal of Physiological Sciences. 2014;64(4):221-32. doi: 10.1007/s12576-014-0314-y.

37. Korte FS, Feest ER, Razumova MV, Tu A-Y, Regnier M. Enhanced Ca2+ binding of cardiac troponin reduces sarcomere length dependence of contractile activation independently of strong crossbridges. American Journal of Physiology - Heart and Circulatory Physiology. 2012;303(7):H863-H70. doi: 10.1152/ajpheart.00395.2012.

38. Lee E-J, Nedrud J, Schemmel P, Gotthardt M, Irving TC, Granzier HL. Calcium sensitivity and myofilament lattice structure in titin N2B KO mice. Archives of Biochemistry and Biophysics. 2013;535(1):7683. doi: http://dx.doi.org/10.1016/j.abb.2012.12.004.

39. Brandt PW, Diamond MS, Rutchik JS, Schachat FH. Co-operative interactions between troponintropomyosin units extend the length of the thin filament in skeletal muscle. Journal of Molecular Biology. 1987;195(4):885-96. doi: http://dx.doi.org/10.1016/0022-2836(87)90492-X. 
40. Land S, Niederer SA. A Spatially Detailed Model of Isometric Contraction Based on Competitive Binding of Troponin I Explains Cooperative Interactions between Tropomyosin and Crossbridges. PLoS Computational Biology. 2015;11(8):e1004376. doi: 10.1371/journal.pcbi.1004376. PubMed PMID: PMC4532474.

41. Izakov V, Katsnelson LB, Blyakhman FA, Markhasin VS, Shklyar TF. Cooperative effects due to calcium binding by troponin and their consequences for contraction and relaxation of cardiac muscle under various conditions of mechanical loading. Circulation Research. 1991;69(5):1171-84. doi: 10.1161/01.res.69.5.1171.

42. Landesberg A, Sideman S. Mechanical regulation of cardiac muscle by coupling calcium kinetics with cross-bridge cycling: a dynamic model1994 1994-08-01 00:00:00. H779-H95 p. 



\title{
Chapter 3
}

\section{High tension in sarcomeres hinders myocardial relaxation: a computational study}

This chapter is based on:

Lauren J. Dupuis, Joost Lumens, Theo Arts, and Tammo Delhaas (2018). High tension in sarcomeres hinders myocardial relaxation: A computational study. In PLoS One. (Accepted).

\begin{abstract}
Experiments have shown that the relaxation phase of cardiac sarcomeres during an isometric twitch is prolonged in muscles that reached a higher peak tension. However, the mechanism is not completely understood. We hypothesize that the binding of calcium to troponin is enhanced by the tension in the thin filament, thus contributing to the prolongation of contraction upon higher peak tension generation. To test this hypothesis, we developed a computational model of sarcomere mechanics that incorporates tension-dependence of calcium binding. The model was used to simulate isometric twitch experiments with time dependency in the form of a two-state crossbridge cycle model and a transient intracellular calcium concentration. In the simulations, peak isometric twitch tension appeared to increase linearly by $51.1 \mathrm{KPa}$ with sarcomere length from $1.9 \mu \mathrm{m}$ to $2.2 \mu \mathrm{m}$. Experiments showed an increase of 47.3 $\mathrm{KPa}$ over the same range of sarcomere lengths. The duration of the twitch also increased with both sarcomere length and peak intracellular calcium concentration, likely to be induced by the inherently coupled increase of the peak tension in the thin
\end{abstract}


filament. In the model simulations, the time to $50 \%$ relaxation $\left(t_{R 50}\right)$ increased over the range of sarcomere lengths from $1.9 \mu \mathrm{m}$ to $2.2 \mu \mathrm{m}$ by $0.11 \mathrm{~s}$, comparable to the increased duration of $0.12 \mathrm{~s}$ shown in experiments. Model simulated $t_{R 50}$ increased by $0.12 \mathrm{~s}$ over the range of peak intracellular calcium concentrations from $0.87 \mu \mathrm{M}$ to 1.45 $\mu \mathrm{M}$. Our simulation results suggest that the prolongation of contraction at higher tension is a result of the tighter binding of $\mathrm{Ca}^{2+}$ to troponin in areas under higher tension, thus delaying the deactivation of the troponin.

\section{Introduction}

Cardiac muscle contraction is triggered by the rise in the concentration of intracellular calcium (1). It has been shown in skinned muscle preparations that a relatively small increase in the free intracellular calcium concentration, i.e. $\left[\mathrm{Ca}^{2+}\right]$, results in disproportionately large increases in steady state tension development, a process that has been called cooperativity in sarcomere activation and contraction $(2,3)$. In literature, researchers generally use a Hill coefficient $\left(n_{\text {Hill }}\right)$ to quantify cooperativity by determining the steepness of the slope in the $\left[\mathrm{Ca}^{2+}\right]$-tension relationship. Sun et al. have found that cooperativity is on the order of $n_{\text {Hill }}=3$ under zero load conditions (4). The data of Dobesh et al. has shown that in the presence of mechanical load, $n_{\text {Hill }}$ approaches a value of 7 (2). This increase of cooperativity suggests that tension in the thin filaments promotes the cooperative effect during sarcomere contraction.

Recently with the MechChem model, we introduced the hypothesis that tension in the thin filament tightens the binding of $\mathrm{Ca}^{2+}$ to the troponin complex (Tn) (5). Model simulations showed that high tension boosts the cooperativity from the purely chemical cooperativity ( $\mathrm{Ca}^{2+}$ binding to $\mathrm{Tn}$ in the absence of a mechanical load) shown by Sun et al. (4) to the cooperativity shown by Dobesh et al. (2) when the thin filament is under tension. The agreement between simulations and experimental data (5) suggested that the proposed tension-dependence of cooperativity may be responsible for the added cooperative activation of $T n^{\prime}$ s. 
Experiments have been conducted to release the afterload during an isometric twitch resulting in a quick drop in sarcomere tension $(6,7)$. The drop in sarcomere tension corresponds with an instantaneous increase in the free intracellular calcium concentration. The immediate increase in free intracellular calcium concentration with a sudden decrease in sarcomere tension shows that the mechanics of the sarcomere has a clear influence on the binding of $\mathrm{Ca}^{2+}$ to $\mathrm{Tn}$. Hence, it is likely that the tensiondependent activation of the $T n^{\prime}$ 's along the thin filament has an effect on the relaxation of cardiac muscle. It has been shown by Janssen and Hunter (8) that the duration of muscle contraction increases linearly with the developed peak tension. While Janssen and Hunter proposed that higher peak tension caused the longer duration of contraction, the underlying physical mechanism that explains the experimental results was lacking. In the present study, we hypothesize that prolongation of contraction, or delay in relaxation, is a result of hindrance to thin filament deactivation imposed by high tension locally in the thin filament. In order to test this hypothesis, we extend the MechChem model, originally designed to simulate steady state tension, with a two state cross-bridge cycle model and a transient intracellular calcium concentration. The additions to the model allow us to simulate isometric twitch contractions. We simulate an isometric twitch at multiple sarcomere lengths $\left(L_{\text {sarc }}\right)$ and compare the model results to the experimental data presented by Janssen and Hunter (8). Additionally, we simulate an isometric twitch with varying peak intracellular calcium concentrations to mimic the experimental conditions of Kassiri et al (9).

\section{Methods}

The previous version of the MechChem model was developed to describe a steady state isometric contraction. In that model, we assumed that $\mathrm{Ca}^{2+}$ binding to the $\mathrm{Tn}$ results in the movement of tropomyosin $(\mathrm{Tm})$ out of the position in which it blocks the binding sites on the thin filament. The binding of $\mathrm{Ca}^{2+}$ to $\mathrm{Tn}$ is cooperative in nature with a baseline chemical cooperativity that is increased by mechanical tension in the thin filament. Additionally, it has been assumed that unblocking of cross-bridge (XB) binding sites implies automatic $X B$ formation and force generation. Furthermore, we 
assumed that all $X B^{\prime}$ s form only in the single overlap region and generate equal force. Tension in the thin filament increases at each location of $X B$ binding by the corresponding $X B$ force. Thus, tension increases step by step from the beginning of the single overlap region near the center of the sarcomere towards the ending of this region near the z-disk.

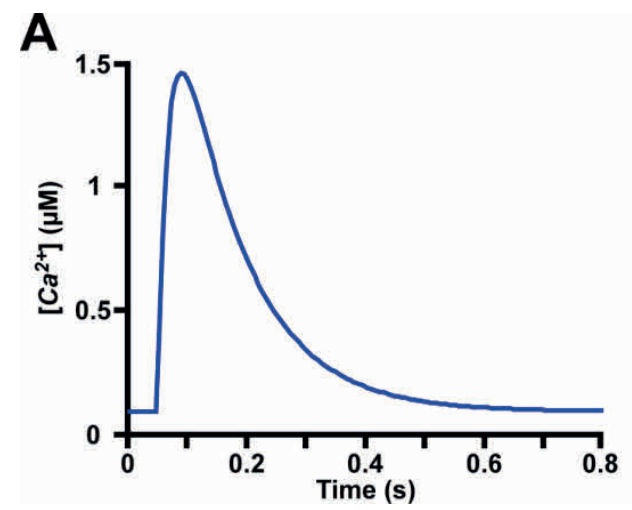

B

Figure 3.1: Schematic of the MechChem Model. (A) The displayed calcium transient derived from Rice et al. (10) is used as an input to the model. (B). Activation of the thin filament, moving from the non-permissive state $(N(x, t))$ to the permissive state $(P(x, t))$ occurs when calcium binds to the $T n$. Myosin heads in the detached, non-force-generating state $(D(x, t))$ can only enter the bound, force-generating cross-bridge state $(A(x, t))$ if the $X B$ binding sites are free for binding. Hence, $P(x, t)$ increases the rate of cross-bridge attachment $\left(f_{D A}\right)$. $A(x, t)$ determines the cross-bridge force density and hence the tension $(S(x, t))$ in the thin filament. The tension suppresses the rate of detachment of $\mathrm{Ca}^{2+}$ from $\mathrm{Tn}\left(f_{N P}\right)$. Solid arrows represent state transition rates while the red dashed arrows indicate effects on state transition rates.

In this study, we have modified the MechChem model of the cardiac sarcomere to mimic isometric twitch conditions. One of the steady state model assumptions detailed by Dupuis et al. (5) has changed, and two assumptions have been added. It is no longer assumed that unblocking of a binding site automatically implies $X B$ binding and force development. Instead, a two state cross-bridge cycle model has been implemented where $\mathrm{Ca}^{2+}$ binding to $T n$ unblocks the binding sites, enabling $X B$ formation with a given reaction rate (Figure 3.1B). One new assumption is that the intracellular $\mathrm{Ca}^{2+}$ concentration varies in time according to the formulation presented 
by Rice et al. (10) (Figure 3.1A). The second new assumption is that the cross-bridge cycle is rate-limiting in comparison with $\mathrm{Ca}^{2+}$ kinetics (11).

At rest, the $T n$ anchors a $T m$ molecule in place blocking the $X B$ binding sites on actin monomers. The binding of a $\mathrm{Ca}^{2+}$ ion to the $\mathrm{Tn}$ triggers a cascade of conformational changes (12). When the $T n$ is activated, the $T m$ strand moves so that the $X B$ binding sites on the thin filament are unblocked (13). We assume that the single overlap region is the only region on the thin filament where $X B^{\prime}$ s can bind. Hence, the working domain of the model is the single overlap region. The position $(x)$ is defined as the position along the single overlap region starting closest to the mid line and ending toward the Z-disk. The mathematical formulation of the proportion $(P(x, t))$ of active $T n^{\prime} s$ at time $t$ and position $x$ along the single overlap region is from the previously developed MechChem model (Equation 3.1). Parameters $C_{S}$ and $K_{\text {TnCaO }}$ represent the tension sensitivity constant of $\mathrm{Tn}$ and an equilibrium $\left[\mathrm{Ca}^{2+}\right]$ constant, respectively.

$$
P(x, t)=\frac{1}{1+e^{n\left(-C_{S} S(x, t)-\ln \left(\frac{\left[C a^{2+}\right](t)}{K_{\text {TnCa }}}\right)\right)}}
$$

Parameter $n$ indicates the Hill coefficient of $\mathrm{Ca}^{2+}$ binding to $\mathrm{Tn}$ in the absence of thin filament tension. We previously found that parameter $K_{\text {TnCaO }}$ changes with $L_{\text {sarc }}(5)$. We now assume $K_{\text {TnCaO }}$ to be linearly related to $L_{\text {sarc }}$ (Equation 3.2).

$$
K_{\text {TnCa0 }}=-a L_{\text {sarc }}+b
$$

Parameters $a$ and $b$ represent the slope and intercept of the linear relation.

The $X B$ cycle model utilized in our study is based on the $X B$ cycle model by Landesberg and Sideman (14). We simplified the model (Figure 3.1B) to a two state model that consisted of a force generating attached state and a non-force generating state being quantified by the fractions $A(x, t)$ and $D(x, t)=1-A(x, t)$, respectively. The rate of $X B$ attachment is assumed to be proportional to the fraction $P(x, t)$ of activated $T n$ complexes. Thus, for the rate of $X B$ increase, the change in $A(x, t)$ with respect to time is represented with Equation 3.3. 


$$
\frac{\partial A(x, t)}{\partial t}=(1-A(x, t)) P(x, t) f_{D A}-A(x, t) f_{A D}
$$

In the original MechChem model developed by Dupuis et al. (5), the tension in the thin filament was defined as the sum of all of the $X B$ forces from the start of the single overlap region near the mid-line till the end of the single overlap region closest to the z-disk. The model represents a population of sarcomeres. Instead of explicit modeling of individual $X B^{\prime} s, X B$ force density is represented as a continuous function of position $x$, proportional with $X B$ fraction $A(x, t)$.

Thus, the spatial derivative of thin filament tension $(\partial S(x, t) / \partial x)$ is represented by Equation 3.4.

$$
\frac{\partial S(x, t)}{\partial x}=C_{f} A_{F}(x, t)
$$

with boundary condition: $S(0, t)=0$

The constant $\left(C_{f}\right)$ represents the tension per unit length in the thin filament if all $X B^{\prime} S$ are active. The length of the single overlap region depends on $L_{\text {sarc }}$ using the formulation of Rice et al. (10) that can be found in the appendix.

\section{Numerical Implementation}

Equation 3.3 was solved with the following method. $A(x, t)$ was represented by an array of samples along the $x$ axis, being used as state variables $\left(A_{i x}\right)$ that varied in time. From the state variables $A_{i x}$, their time derivatives are calculated as follows. Tension $S(x, t)$ is calculated as $S_{i x}$ for each sample $A_{i x}$, applying Equation 3.4. Thus, the numerical integration along the $x$-direction is carried out by the cumulative summing of $A_{i x}$. From the tension samples $S_{i x}, T n$-activation $P_{i x}$ is calculated using Equation 3.1. From Tnactivation $P_{i x}$, the time derivative of $X B$ density $\partial A_{i x} / \partial t$ is calculated using Equation 3.3. Having written $\partial A_{i x} / \partial t$ as a function of $A_{i x}$, the related differential equation is solved by utilizing the Matlab ode23s solver. 


\section{Parameter estimation and Simulation Protocol}

Figure 2 from Janssen and Hunter, 1995 (8) was discretized, and data points were extracted from each curve at time points spaced every 0.02 seconds. The parameters $C_{s}, C_{f}, a$, and $b$ have been estimated by minimizing the error between the modelgenerated thin filament tension $\left(S_{\text {model }}\right)$ and the experimental data from Janssen and Hunter $\left(S_{\text {experiment }}\right)$. The objective function used to calculate the error is shown in Equation 3.5.

$$
\text { error }=\frac{\sum_{i=1}^{j}\left(S_{\text {model }}-S_{\text {experiment }}\right)^{2}}{j}
$$

\section{Simulation Protocol}

Simulations were designed to mimic the isometric twitch experiments of Janssen and Hunter. $L_{\text {sarc }}$ was held constant given parameter values $a, b, C_{f}$, and $C_{s}$, while calcium concentration changed in time with a peak value of $1.45 \mu \mathrm{M}$. The model provided thin filament tension as a function of time for 7 values of $L_{\text {sarc }}$ ranging from 1.90 to $2.20 \mu \mathrm{m}$. Additionally, simulations were performed in which $L_{\text {sarc }}$ was maintained at $2.2 \mu \mathrm{m}$ while calcium gradients were varied as follows: the amplitude of the calcium concentration was increased stepwise from $60 \%$ to $100 \%$ of the peak value used in the other simulations while the time constants of rise and decay remained constant. This simulation protocol mimics the experiments of Kassiri et al (9), showing that twitch duration increases with peak force independently from sarcomere length.

\section{Results}

The full set of parameters utilized is displayed in Table 3.1. The parameter values resulting from the fitting procedure are $a, b, C_{f}$, and $C_{s}$.

The comparison between data points from the experimental isometric twitch tension curves of Janssen and Hunter (8) and the model-generated tension curves is shown in Figures 3.2A and 3.2B. The ratio of the peak tension at $L_{\text {sarc }} 1.9 \mu \mathrm{m}$ to that measured at $L_{\text {sarc }} 2.2 \mu \mathrm{m}$ was 0.48 in experiments and 0.42 in simulations. 


\begin{tabular}{|c|c|c|c|c|}
\hline Parameter & Value & Units & Equation & Source \\
\hline$a$ & 1.39 & $\mu \mathrm{M} \mu \mathrm{m}^{-1}$ & 3.2 & model fit \\
\hline$b$ & 7.76 & $\mu \mathrm{M}$ & 3.2 & model fit \\
\hline$f_{D A}$ & 40 & $\mathrm{~s}^{-1}$ & 3.3 & (14) \\
\hline$C_{f}$ & 0.283 & $\mathrm{kPa} \mathrm{nm}^{-1}$ & 3.4 & model fit \\
\hline$C_{S}$ & 0.127 & $\mathrm{kPa}^{-1}$ & 3.1 & model fit \\
\hline$n$ & 3 & unitless & 3.1 & (4) \\
\hline$f_{A D}$ & 12 & $\mathrm{~s}^{-1}$ & 3.3 & (14) \\
\hline
\end{tabular}
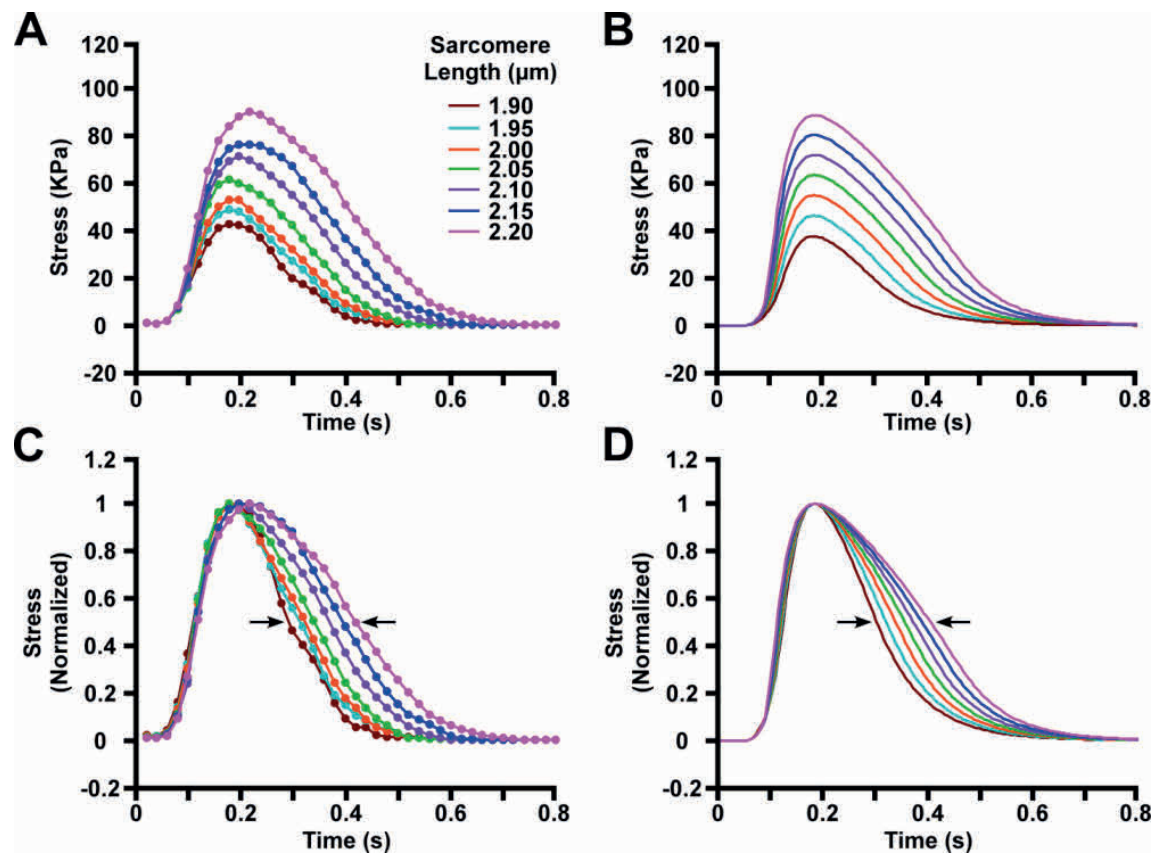

Figure 3.2: Tension traces of Sarcomeres in isometric conditions. (A) Experimentally measured tension traces at $L_{\text {sarc }}$ ranging from 1.90 to $2.20 \mu \mathrm{m}$ under isometric conditions. The dots are data points extracted from Figure 2 of Janssen and Hunter 1995 (8). (B) Model simulated tension traces mimicking the conditions in A are displayed. (C) The experimentally measured tension data in A are each normalized to their own peak tension. The arrows in the figure represent the relaxation to $50 \%$ of the maximum stress level after the peak stress. (D) The model-generated tension traces in B are each normalized to their own peak tension. 
Figures $3.2 \mathrm{C}$ and 3.2D display the tension traces of Figures $3.2 \mathrm{~A}$ and $3.2 \mathrm{~B}$, respectively, but each curve is normalized to its own peak tension. From these normalized curves, the prolongation of tension generation is obvious at larger $L_{\text {sarc }}$ 's. The time from peak tension generation to $50 \%$ relaxation $\left(t_{R 50}\right)$ differs between the largest $\left(L_{\text {sarc }}=2.2 \mu \mathrm{m}\right)$ and the smallest $\left(L_{\text {sarc }}=1.9 \mu \mathrm{m}\right) L_{\text {sarc }}$ by 0.12 seconds in experiments and 0.11 seconds in simulations. The $t_{R 50}$ for the sarcomere lengths ranging from $1.9 \mu \mathrm{m}$ to $2.2 \mu \mathrm{m}$ is shown in Figure 3.3. Additionally, the peak tension generated by the model has been compared to the experimental values from Janssen and Hunter (8) in Figure 3.3.

The model predicted proportion of $X B^{\prime} S$ in the force generating state $(A)$ during an isometric twitch as a function of time $(t)$ and position $(x)$ at $L_{\text {sarc }}=2.05 \mu \mathrm{m}$ is shown in Figure 3.4. The $L_{\text {sarc }}$ of $2.05 \mu \mathrm{m}$ was chosen because it was in the middle of the range of $L_{\text {sarc }}$ 's. The model predicts that the highest likelihood of $X B^{\prime}$ 's to generate force occurs for high $x$-values, i.e. in the single overlap region closest to the z-disk. Tension development at position $x$ in the thin filament is prolonged in the single overlap region near the z-disk as compared to this region near the center of the sarcomere. At $x=400$ $\mathrm{nm}$, the probability that there will be a strong cross-bridge bound reaches its peak value at a time of $0.20 \mathrm{~s}$ (marker 1 ). The probability of a bound cross-bridge drops to 0.10 by $0.38 \mathrm{~s}$ (marker 2). However, at the boundary of the single overlap region near the z-disk $(x=625 \mathrm{~nm}$ ), the probability of cross-bridge formation reaches its peak value of 0.77 at $0.31 \mathrm{~s}$ (marker 3 ) and drops to 0.10 at $0.56 \mathrm{~s}$ (marker 4). The time difference between markers 2 and 4 shows that relaxation of the thin filament is delayed in the area under the highest tension, i.e. in the single overlap region near the z-disk. 


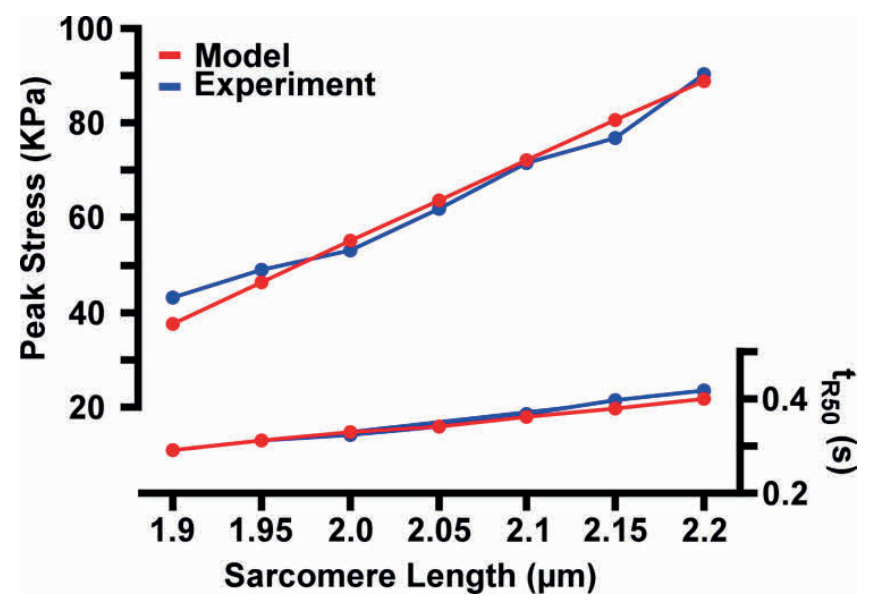

Figure 3.3: Comparison of model predicted metrics with experimental results. The peak tension and the time at which the muscle has relaxed by $50 \%\left(t_{R 50}\right)$ for each $L_{\text {sarc }}$ are compared between the model prediction (red) and the experimental results (blue).

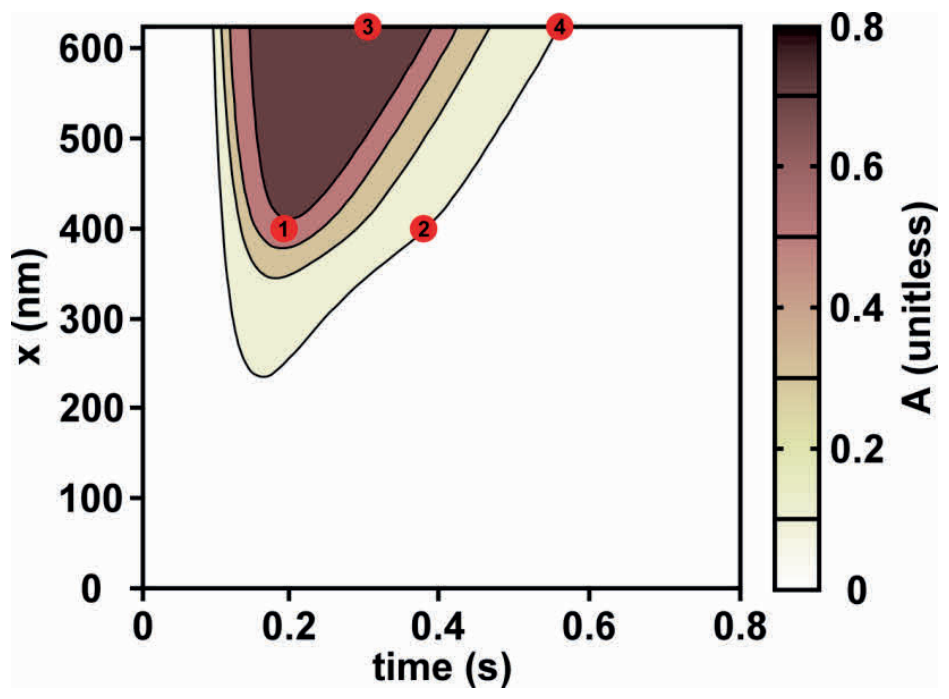

Figure 3.4: Proportion of force-generating cross-bridges $(A)$ throughout an isometric twitch. $A$ is displayed for sarcomere with length $2.05 \mu \mathrm{m}$ under isometric twitch conditions. The probability of formation of a force-generating cross-bridge depends both on time and position $(x)$ along the single overlap region of the thin filament. Markers 1 and 2, located at $400 \mathrm{~nm}$ from the mid line, show the peak level of activation of 0.65 and when the activation level reaches 0.10, respectively. Markers 3 and 4, located at $625 \mathrm{~nm}$ from the mid line closest to the z-disk, show the peak level of activation 0.77 and the subsequent drop to 0.10 , respectively. 
Figure 3.5 displays myofiber isometric twitch stress curves (magenta) resulting from input calcium transients (blue). The peak intracellular calcium concentration was increased from $0.87 \mu \mathrm{M}$ to $1.45 \mu \mathrm{M}$. The $t_{R 50}$ for the lowest peak intracellular calcium concentration was $0.10 \mathrm{~s}$, increasing to $0.22 \mathrm{~s}$ when the peak intracellular calcium concentration was increased to $1.45 \mu \mathrm{M}$. With greater peak tension, the duration of contraction increased.
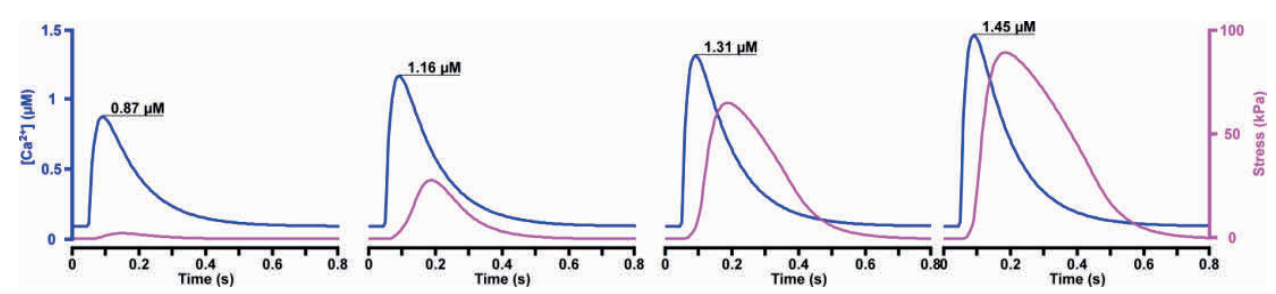

Figure 3.5: Isometric twitch stress with increases in peak $\left[\mathrm{Ca}^{2+}\right]$. The input calcium transient (blue curves) increases its peak value from $0.87 \mu \mathrm{M}$ to $1.45 \mu \mathrm{M}$ while maintaining time constants of rise and decay. The related isometric twitch stress curves (magenta) are shown for a sarcomere length of $2.2 \mu \mathrm{m}$.

\section{Discussion}

To study the time course of force development and relaxation of cardiac sarcomere contraction, we incorporated the dynamics of the cross-bridge cycle in our previously developed MechChem model of the mechano-chemical interactions in cardiac troponin activation (5). In addition to the assumed tension-dependent calcium-binding cooperativity in this model, we implemented time dependence by means of imposed calcium transients. We simulated isometric twitches for a range of sarcomere lengths and compared our results to the experimental data of Janssen and Hunter and Kassiri et al. $(8,9)$. Our model with only six parameters and two partial differential equations could reproduce the isometric twitch behavior as evidenced by strikingly similar values for each sarcomere length between model and experiment for peak tension, time of $50 \%$ relaxation, and increases in twitch duration with increased peak intracellular calcium concentration. These results not only suggest that the resultant force development after binding of calcium to troponin favors new bindings of calcium to troponin, but also that hindrance to thin filament deactivation increases with tension 
in the thin filament. The resultant of both mechanisms is an increase of both peak isometric tension and duration of the twitch with sarcomere length and peak intracellular calcium concentration. An interesting consequence of our hypothesis on tension-dependency of calcium binding to troponin is that contraction duration is locally distributed within a sarcomere, with longest duration close to the z-disk where tension in the thin filament is highest.

\section{Intracellular calcium transient}

There are multiple channels, pumps, and buffers that determine the intracellular calcium transient in cardiac muscle (1). For simplicity we have utilized the prescribed calcium transient formulated by Rice et al. (10) as the input in this study. While calcium handling is tightly controlled in cardiac muscle, there are pathological situations that cause differences in calcium transient morphology (15). A model of the calcium handling system will be necessary when studying a pathological situation, but the prescribed transient is suitable for our study in which we investigate the isometric twitch in healthy tissue at different sarcomere lengths. While a change in sarcomere length has an acute effect on the calcium transient, a recent article (16) has shown that intracellular calcium transient morphology does not exhibit significant long term changes in rat ventricular myocytes with a change in cell length.

\section{Rise of tension}

Experiments have shown that the upslope of tension in isometric twitches decreases with sarcomere length $(8,17)$. The MechChem model simulated upslope of tension development is independent of sarcomere length. It is possible that addition of a third cross-bridge state, a weakly-bound, non-force-generating state, could alter the rate of rise of tension in the sarcomere. It is also possible that the assumed calcium transient morphology is inconsistent with that in the experiments of Janssen and Hunter (8). Unfortunately, the free intracellular calcium concentration was not measured in the experiments. Janssen and Hunter altered the extracellular calcium concentration and did not measure the intracellular concentrations of calcium. Hence, we assumed a calcium transient in the absence of exact measurements. 


\section{Linear increase of peak tension with sarcomere length}

Experiments have shown that the peak isometric twitch tension developed in the sarcomere increases with sarcomere length $(8,18)$. The results of the MechChem model show a similar linear relationship between peak tension and sarcomere length. The increase in peak tension with sarcomere length shown in our simulations is a result of the increasing length of the single overlap region and the mechanism of cooperative activation as described by the MechChem model (5). We are aware that there are many proposed mechanisms of cooperative activation in cardiac muscle including nearest neighbor interactions (19), myofilament lattice spacing (20), the cooperative realignment of binding sites (21), the interaction between mutually overlapping $T m^{\prime}$ 's under $T n^{\prime} s(22)$, and the positive feedback between the number of cross-bridges and the binding affinity of $\mathrm{Ca}^{2+}$ for $\mathrm{Tn}(23)$, just to name a few. We previously proposed that the affinity of $\mathrm{Ca}^{2+}$ binding to $\mathrm{Tn}$ is boosted by mechanical tension locally in the thin filament. The cooperative mechanism we proposed is consistent with the results of previous studies suggesting that $\mathrm{Ca}^{2+}$ binding to $\mathrm{Tn}$ tightens with sarcomere tension (17). Hence, the cooperative activation mechanism presented in our previous study (5) was conserved within the current model framework. However, parameters $C_{s}$ and $C_{f}$ changed values because of the different experimental circumstances (3). With the addition of the $X B$ cycle, we have presented a model of the cardiac sarcomere that is composed of 2 partial differential equations and six parameters that is able to reproduce a range of isometric twitch conditions without further change of parameter values.

\section{Increase of peak tension with peak intracellular calcium concentration}

Increases in peak intracellular calcium concentration result in increases in peak myofiber tension in both steady state isometric experiments (2) and isometric twitch experiments (9). In the MechChem model, the affinity of $\mathrm{Tn}$ to bind $\mathrm{Ca}^{2+}$ increases with $L_{\text {sarc }}$. Therefore, some of the increases in twitch duration shown at high $L_{\text {sarc }}$ can be due to the increased affinity for $\mathrm{Ca}^{2+}$ instead of solely due to the increased peak tension. To uncouple the increase in tension generation from increased $\mathrm{Ca}^{2+}$ affinity, we have 
mimicked the experimental conditions of Kassiri et al. by maintaining the $L_{\text {sarc }}$ at 2.2 $\mu \mathrm{m}$ while altering the peak intracellular calcium concentration. The working range of calcium concentrations used by Kassiri et al. in experiments was lower than that used in our model, so relative changes were utilized for comparison, and the amplitude of the intracellular calcium transient was increased stepwise from $60 \%$ to $100 \%$ of the peak value used in our simulations. With greater peak intracellular calcium concentration, tension in the thin filament increased. The twitch duration increased with peak tension independent of the $L_{\text {sarc }}$ in these simulations. The additional test of the model provides further results supporting our hypothesis that greater tension in the thin filament locally hinders relaxation.

\section{Tension dependent mechanism of relaxation in cardiac muscle}

The experiments of Janssen and Hunter (8) have shown that cardiac myocyte relaxation during an isometric twitch is prolonged with greater developed peak tension. However, a likely physical mechanism that causes the prolongation of contraction is not described yet. A recent review by Biesiadecki et al. (24) highlights three possible rates that determine the moment and rate of relaxation: intracellular calcium decline, troponin deactivation, and cross-bridge cycling rates. Each of the three rates mentioned contribute to relaxation morphology.

Many computational models have been developed that replicate the prolongation of contraction with greater peak tension generated in the cardiac sarcomere. Landesberg and Sideman (14) developed a model of the cardiac sarcomere including $T n$ activation and the $X B$ cycle. They propose that the number of strongly bound $X B^{\prime}$ s increases the affinity of $\mathrm{Tn}$ to bind $\mathrm{Ca}^{2+}$. Similarly, Niederer et al. proposed a thorough mathematical description of the cardiac sarcomere to understand relaxation of cardiac muscle (25). The tension developed in the sarcomere by the Niederer model decreases the rate of detachment of $\mathrm{Ca}^{2+}$ from $\mathrm{Tn}$. While both the Niederer and the Landesberg and Sideman models propose cooperativity mechanisms dependent on sarcomere tension, an underlying physical explanation is lacking. They claim interactions between $T n^{\prime}$ s on the thin filaments over distances as large as $35 \mathrm{~nm}$ but do not provide a firm physical 
basis for this interaction. With the MechChem model, we propose a physico-chemical mechanism that tightens $\mathrm{Ca}^{2+}$ binding to $\mathrm{Tn}$ by molecular deformation caused by mechanical tension in the thin filaments, finally resulting in prolongation of contraction at higher peak tension. By incorporating the spatial scale along the thin filament, tension in the thin filament changes locally with position $x$. Greater tension in the thin filament renders release of $\mathrm{Ca}^{2+}$ from $\mathrm{Tn}$ energetically less favorable. Consequently, high tension in the thin filament impedes relaxation.

The model developed by Rice et al. (10) is able to reproduce sarcomere mechanics including isometric twitches and isotonic contractions utilizing a system of ordinary differential equations (ODE). The prolongation of contraction at higher $L_{\text {sarc }}$ 's is present within the results of the Rice model. The latter authors assume a mathematical formulation that the parameter governing the rate of transition from the weakly bound, non-force-generating $X B$ state to the unbound $X B$ state increases at lower $L_{\text {sarc }}$ 's. Consequently, the prolongation of contraction is, at least in part, prescribed by the parameter formulation. However, the mechanism behind the increasing rate transition rate at lower $L_{\text {sarc }}$ 's is unclear.

We propose that high tension increases the strength of the binding between $\mathrm{Ca}^{2+}$ and $\mathrm{Tn}$, thereby hindering relaxation. In our simulations, relaxation begins in areas of the single overlap region closer to the midline where tension is lower. Even if tension in the thin filament at the z-disk is at its maximum, there is always a relatively loose end closest to the sarcomere mid line under no tension. Consequently, as the intracellular calcium concentration decreases, relaxation will begin at this loose end first.

Our model simulations suggest heterogeneity in $X B$ binding along the single overlap region caused by the gradient of tension along the thin filament. This directionality in $X B$ binding was not found by Desai and colleagues (26), who fluorescently labeled S1 myosin heads to view individual bindings to an actin monomer on a strand. However, the myosin heads in that study were free in solution, so there was no force generated. According to our hypothesis that thin filament tension enhances $\mathrm{Ca}^{2+}$ binding to $\mathrm{Tn}$, we 
would not expect directionality to cross-bridge binding when the thin filament is not subject to mechanical load. The hypothesis we propose could potentially be tested if the experimental setup of Desai could be altered to incorporate force generating myosin heads.

\section{Limitations}

The MechChem model is a relatively simple model that utilizes two partial differential equations and six parameters to characterize the isometric twitch contraction of a cardiac sarcomere. Due to the simplicity of the MechChem model, some mechanisms that impact the cooperative binding of $\mathrm{Ca}^{2+}$ to $\mathrm{Tn}$ may not be included. Additionally, a prescribed calcium transient has been imposed as an input to the model, so force feedback on the electrophysiology (27) is currently not included. However, the calcium transient is an input that can later be replaced with a model. In the study by Janssen and Hunter (8) intracellular $\left[\mathrm{Ca}^{2+}\right]$ was not measured. Hence, we had to make assumptions on the calcium transient. Additionally, we have utilized the assumption that $\left[\mathrm{Ca}^{2+}\right]$ is uniform throughout the entire sarcomere lattice and the cell. It has been shown that in large animals such as pigs, there are intracellular spatial inhomogeneities in the $\mathrm{Ca}^{2+}$ transient (28). However, mice did not display inhomogeneity in $\left[\mathrm{Ca}^{2+}\right]$ probably due to the high density of T-tubules. Hence, the assumption of $\left[\mathrm{Ca}^{2+}\right]$ homogeneity is likely relatively accurate when modeling rat cardiomyocytes but may be incorrect when modeling a larger animal or human. Additionally, the calcium dynamics controlling the activation of the thin filament in the MechChem model are currently represented with the steady state calculation of $P(x, t)$. The use of steady state calcium binding rather than a dynamic description is considered a reasonable assumption because the cross-bridge cycle has been shown to be rate limiting. Therefore, the calcium dynamics can be viewed in equilibrium. When sarcomere shortening is added to the model, a description of the dynamics of calcium binding will be necessary.

The MechChem XB cycle model is a simplified, two state model. Although the twostate $X B$ cycle used in our model is a simplified representation of a more complex 
biological system, the lumping of these states improves the computational expense of running such a model while still providing sufficient complexity necessary to test our hypothesis.

\section{Conclusion}

The MechChem model of cardiac sarcomere contraction has been extended with a XB cycle model and a calcium transient that changes in time to simulate an isometric twitch at multiple sarcomere lengths. The results of the MechChem model showed that peak isometric twitch tension and the duration of the twitch increased with sarcomere length. The results support our hypothesis that high tension in the thin filament locally hinders relaxation. Compared to other models of cardiac sarcomere isometric twitch contraction, the MechChem model is simple with few parameters while many properties of myocyte contraction are included. 


\section{References}

1. Bers DM. Calcium Fluxes Involved in Control of Cardiac Myocyte Contraction. Circulation Research. 2000;87(4):275-81.

2. Dobesh DP, Konhilas JP, de Tombe PP. Cooperative activation in cardiac muscle: impact of sarcomere length. American Journal of Physiology - Heart and Circulatory Physiology. 2002;282(3):H1055H62.

3. Kentish JC, ter Keurs HE, Ricciardi L, Bucx JJ, Noble MI. Comparison between the sarcomere length-force relations of intact and skinned trabeculae from rat right ventricle. Influence of calcium concentrations on these relations. Circulation Research. 1986;58(6):755-68.

4. Sun Y-B, Lou F, Irving M. Calcium- and myosin-dependent changes in troponin structure during activation of heart muscle. The Journal of Physiology. 2009;587(Pt 1):155-63.

5. Dupuis L, Lumens J, Arts T, Delhaas T. Mechano-chemical Interactions in Cardiac Sarcomere Contraction: A Computational Modeling Study. PLOS Computational Biology. 2016;12(10):e1005126.

6. Backx PH, Ter Keurs HE. Fluorescent properties of rat cardiac trabeculae microinjected with fura2 salt. American Journal of Physiology - Heart and Circulatory Physiology. 1993;264(4):H1098-H110.

7. Kurihara S, Komukai K. Tension-dependent changes of the intracellular $\mathrm{Ca} 2+$ transients in ferret ventricular muscles. The Journal of Physiology. 1995;489(Pt 3):617-25.

8. Janssen PM, Hunter WC. Force, not sarcomere length, correlates with prolongation of isosarcometric contraction. American Journal of Physiology. 1995;269(2):H676-H85.

9. Kassiri Z, Myers R, Kaprielian R, Banijamali HS, Backx PH. Rate-dependent changes of twitch force duration in rat cardiac trabeculae: a property of the contractile system. The Journal of Physiology. 2000;524(Pt 1):221-31.

10. Rice JJ, Wang F, Bers DM, de Tombe PP. Approximate Model of Cooperative Activation and Crossbridge Cycling in Cardiac Muscle Using Ordinary Differential Equations. Biophysical Journal. 2008;95(5):2368-90.

11. Janssen PML, Stull LB, Marbán E. Myofilament properties comprise the rate-limiting step for cardiac relaxation at body temperature in the rat. American Journal of Physiology-Heart and Circulatory Physiology. 2002;282(2):H499-H507.

12. Solaro J. Chapter 30 - Mechanisms Regulating Cardiac Myofilament Response to Calcium. In: Cohen NSKTV, editor. Heart Physiology and Pathophysiology (Fourth Edition). San Diego: Academic Press; 2001. p. 519-26.

13. Solaro RJ, Rarick HM. Troponin and Tropomyosin: Proteins That Switch on and Tune in the Activity of Cardiac Myofilaments. Circulation Research. 1998;83(5):471-80.

14. Landesberg A, Sideman S. Mechanical regulation of cardiac muscle by coupling calcium kinetics with cross-bridge cycling: a dynamic model. American Journal of Physiology. 1994;267(2):H779-H95.

15. Briston SJ, Dibb KM, Solaro RJ, Eisner DA, Trafford AW. Balanced changes in Ca buffering by SERCA and troponin contribute to $\mathrm{Ca}$ handling during $\beta$-adrenergic stimulation in cardiac myocytes. Cardiovascular Research. 2014;104(2):347-54.

16. Schönleitner P, Schotten U, Antoons G. Mechanosensitivity of microdomain calcium signalling in the heart. Progress in Biophysics and Molecular Biology. 2017.

17. Allen DG, Kurihara $\mathrm{S}$. The effects of muscle length on intracellular calcium transients in mammalian cardiac muscle. The Journal of Physiology. 1982;327:79-94.

18. Wannenburg T, Janssen PML, Fan D, De Tombe PP. The Frank-Starling mechanism is not mediated by changes in rate of cross-bridge detachment. American Journal of Physiology - Heart and Circulatory Physiology. 1997;273(5):H2428-H35.

19. Razumova MV, Bukatina AE, Campbell KB. Different Myofilament Nearest-Neighbor Interactions Have Distinctive Effects on Contractile Behavior. Biophysical Journal. 2000;78(6):3120-37.

20. Williams CD, Salcedo MK, Irving TC, Regnier M, Daniel TL. The length-tension curve in muscle depends on lattice spacing. Proceedings of the Royal Society B: Biological Sciences. 2013;280(1766):20130697.

21. Daniel TL, Trimble AC, Bryant Chase P. Compliant Realignment of Binding Sites in Muscle: Transient Behavior and Mechanical Tuning. Biophysical Journal. 1998;74(4):1611-21.

22. Campbell SG, Lionetti FV, Campbell KS, McCulloch AD. Coupling of Adjacent Tropomyosins Enhances Cross-Bridge-Mediated Cooperative Activation in a Markov Model of the Cardiac Thin Filament. Biophysical Journal. 2010;98(10):2254-64. 
23. Izakov V, Katsnelson LB, Blyakhman FA, Markhasin VS, Shklyar TF. Cooperative effects due to calcium binding by troponin and their consequences for contraction and relaxation of cardiac muscle under various conditions of mechanical loading. Circulation Research. 1991;69(5):1171-84.

24. Biesiadecki BJ, Davis JP, Ziolo MT, Janssen PML. Tri-modal regulation of cardiac muscle relaxation; intracellular calcium decline, thin filament deactivation, and cross-bridge cycling kinetics. Biophysical Reviews. 2014;6(3):273-89.

25. Niederer SA, Hunter PJ, Smith NP. A Quantitative Analysis of Cardiac Myocyte Relaxation: A Simulation Study. Biophysical Journal. 2006;90(5):1697-722.

26. Desai RA, Geeves MA, Kad NM. Using Fluorescent Myosin to Directly Visualize Cooperative Activation of Thin Filaments. Journal of Biological Chemistry. 2014.

27. ter Keurs HEDJ, Shinozaki T, Zhang YM, Zhang ML, Wakayama Y, Sugai Y, et al. Sarcomere mechanics in uniform and non-uniform cardiac muscle: A link between pump function and arrhythmias. Progress in Biophysics and Molecular Biology. 2008;97(2-3):312-31.

28. Heinzel FR, Bito V, Volders PGA, Antoons G, Mubagwa K, Sipido KR. Spatial and Temporal Inhomogeneities During $\mathrm{Ca}^{2+}$ Release From the Sarcoplasmic Reticulum in Pig Ventricular Myocytes. Circulation Research. 2002;91(11):1023-30. 



\title{
Chapter 4
}

\section{Mechano-chemical interactions in cardiac sarcomere shortening}

This chapter is based on:

Lauren J. Dupuis, Joost Lumens, Theo Arts, and Tammo Delhaas (2018). Mechanochemical interactions in cardiac sarcomere shortening. In Proceedings of Computing in Cardiology.

\begin{abstract}
Tension development in the cardiac sarcomere is highly cooperative, yet the mechanism is not known. We have developed the MechChem model of sarcomere mechanics, which incorporates a mechano-chemical interaction-based cooperativity mechanism in which the thin filament has an intrinsic chemical cooperativity that is boosted by mechanical tension in the thin filament.. In the current study, we extend the MechChem model to include the possibility for the sarcomere to change length when contracting against different afterloads. The model was tested at various afterloads and compared to experimental data. The MechChem model successfully reproduced isotonic twitch experiments.
\end{abstract}

\section{Introduction}

The most basic functional unit of cardiac contraction, a sarcomere, is composed of thick and thin filaments. The binding of a calcium ion $\left(\mathrm{Ca}^{2+}\right)$ to a troponin complex $(\mathrm{Tn})$ on the thin filament triggers conformational changes that unblock binding sites (1). 
Cross-bridges $(X B)$ can form and generate force when myosin heads on thick filaments bind to unblocked binding sites on a thin filament. Force generation in the cardiac sarcomere is cooperative, meaning that small increases in the intracellular $\mathrm{Ca}^{2+}$ concentration produce disproportionately large increases in force (2). We have proposed a novel mechanism of cooperativity in which an intrinsic chemical cooperativity in the thin filament is boosted by mechanical tension (3). High tension locally along the thin filament impedes the unbinding of $\mathrm{Ca}^{2+}$ from the $\mathrm{Tn}$, thereby hindering relaxation. Based on this proposed mechanism of cooperativity, we developed the MechChem model of mechano-chemical interactions in cardiac sarcomere contractions (3) that was validated against experimental data on steadystate isometric contraction in skinned muscle (2) and isometric twitches in myofibers $(4,5)$. The aim of this study is to extend the MechChem model to mimic isotonic twitch experiments where the sarcomere can contract and shorten against different afterloads. Model validity will be evaluated by comparison of simulated and measured isotonic twitch experiments at various afterloads.

\section{Methods}

\section{Model Setup}

The setup of the MechChem model is based on several assumptions. First, we assume that binding of $\mathrm{Ca}^{2+}$ to a $\mathrm{Tn}$ results in the movement of tropomyosin ( $T m$ ) away from its rest position, unblocking the $X B$ binding sites on the thin filament. We assume there is an intrinsic chemical cooperativity in the binding of $\mathrm{Ca}^{2+}$ to $\mathrm{Tn}(6)$ that is boosted by mechanical tension in the thin filament. When binding sites are unblocked for $X B$ binding, we assume a XB can either be in the attached or detached states. Each bound $X B$ is assumed to generate a discrete force on the thin filament that sums up to the tension at position $x$ along the single overlap region (SOR) from the start of the mid line to the $z$-disk. The final assumption is that the sarcomere length $\left(L_{\text {sarc }}\right)$ changes with a Hill-type model (7). 
At rest, the binding sites for myosin are blocked by Tm molecules that wrap around the thin filament. They are anchored in place by $\mathrm{Tn}$ complexes. When $\mathrm{Ca}^{2+}$ binds to the $\mathrm{Tn}$, conformational changes occur that result in the movement of $\mathrm{Tm}$ away from the position in which it blocks the binding sites on the thin filament. We refer to this as the activation of the $T n$. The proportion of activated $T n$ at time $t$ and position $x$ along the thin filament, $P(x, t)$, is modeled with the following partial differential equation:

$$
\frac{\partial P(x, t)}{\partial t}=(1-P(x, t))\left(\frac{\left[C a^{2+}\right](t)}{K_{C a}(t)}\right)^{n} f_{C a_{D A}}-f_{C a_{A D}} P(x, t) e^{-n C_{S} S_{x}(x, t)}
$$

The calcium concentration $\left(\left[\mathrm{Ca}^{2+}\right]\right)$ is assumed to be uniform across the cell and to vary in time according to Rice et al. (8). Parameter $n=3$ represents the intrinsic cooperativity of the binding of $\mathrm{Ca}^{2+}$ to $\mathrm{Tn}$ in the absence of tension as described in Sun et al (6). $C_{s}$ represents the tension cooperativity constant. The parameter $f_{C a_{D A}}$ represents the rate of attachment of a $\mathrm{Ca}^{2+}$ to $\mathrm{Tn}$, and $f_{C a}$ represents the rate of detachment of $\mathrm{Ca}^{2+}$ from $\mathrm{Tn} . K_{\mathrm{Ca}}$ is an equilibrium constant that changes linearly with $L_{\text {sarc }}$ according to Equation 4.2 where parameters $a$ and $b$ are constants.

$$
K_{C a}=-a L_{s a r c}+b
$$

Once myosin heads on the thick filaments bind to unblocked sites, XBs can form and generate force. This is only possible in the SOR of the sarcomere, defined as the length of thin filament that overlaps with one thick filament and no other thin filaments. We calculate the length of the SOR as described previously (3).

The probability of formation of a strong $X B$ is represented by $A(x, t)$ (Equation 4.3).

$$
\frac{\partial A(x, t)}{\partial t}=(1-A(x, t)) f_{D A} P(x, t)-A(x, t) f_{A D}
$$

where the rates of $X B$ attachment and detachment are $f_{D A}=40 \mathrm{~s}^{-1}$ and $f_{A D}=12 \mathrm{~s}^{-1}$, respectively (9). 
Because the MechChem model represents an average of thousands of sarcomeres, $A(x, t)$ can be viewed as a density of $X B s$. The force density $\left(F_{X B}(x, t)\right)$ generated by the bound $X B$ s can be represented with Equation 4.4.

$$
F_{X B}(x, t)=F_{i s o} A(x, t) H(t)
$$

In Equation 4.4, $F_{\text {iso }}$ represents the maximum force generated by a single $X B$, and $H(t)$ is a normalized sarcomere length $L_{\text {sarc }}$ determined by Equation 4.5 .

$$
H(t)=\frac{h_{s}(t)-h_{i}(t)}{h_{S E 0}}
$$

We utilize a Hill-type model to represent the changes in length in the sarcomere. The half $L_{\text {sarc }}$ is represented by $h_{s}(\mathrm{t})$, the length of the intrinsic element is $h_{i}(\mathrm{t})$, and the length of the series elastic element where maximum force is generated is $h_{S E O} . H(t)$ is a proportion of the current length of the series elastic element, defined as $h_{s}(t)-h_{i}(t)$ to the length where the series elastic element generates the most tension $\left(h_{S E O}\right)$.

The change in length of the intrinsic element $h_{i}$ is determined with Equation 4.6.

$$
\frac{d h_{i}(t)}{d t}=(H(t)-1) v_{\max }
$$

where parameter $v_{\max }$ refers to the maximum velocity of sarcomere shortening.

The half sarcomere length $h_{s}(t)$ is determined by Equation 4.7.

$$
h_{s}(t)=\min \left\{\begin{array}{c}
h_{\text {iso }} \\
\frac{h_{S E 0}\left(S_{\text {afterload }}-S_{\text {passive }}(t)\right)}{F_{\text {iso }} A_{\text {Xmax }}(t)}+h_{i}(t)
\end{array}\right.
$$

A sarcomere shortens when it develops enough tension at the end of the SOR to overcome the afterload $\left(S_{\text {afterload }}\right)$. The afterload re-stretches the sarcomere when there is no longer enough tension developed to overcome it. If the sarcomere does not change length, $L_{\text {sarc }}$ is held constant at the same length as the previous step $\left(h_{\text {iso }}\right)$. $A_{X \max }$ 
represents the cumulative sum of the $X B$ density at the end of the SOR. $S_{\text {passive }}$ is the total passive tension in the sarcomere.

The output of the MechChem model is the total tension in the thin filament $\left(S_{x}\right)$ at location $x$ along the SOR and time $t$. It is the sum of the total active tension $\left(S_{x a c t i v e}(x, t)\right)$ and the total passive tension $\left(S_{\text {passive }}(t)\right)$ at time $t$, as shown in Equation 4.8.

$$
S_{x}(x, t)=S_{x_{\text {active }}}(x, t)+S_{\text {passive }}(t)
$$

$S_{x_{\text {active }}}(x, t)$ is modeled with Equation 4.9. The $X B$ density, $F_{X B}$, is integrated along the $x$ dimension at each time point to calculate the active tension locally in the thin filament.

$$
\frac{\partial S_{x_{\text {active }}}(x, t)}{\partial x}=F_{X B}(x, t)
$$

$S_{\text {passive }}(t)$ is modeled by the experimental results of Weiwad et al. 2000 (10) (Equation 4.10), depending on $L_{\text {sarc }}$

$$
S_{\text {passive }}=4.41\left(e^{2.87\left(L_{\text {sarc }}-1.85\right)}-1\right)
$$

\section{Parameter fitting procedure}

The parameters $F_{i s o}, C_{s}, a$, and $b$ were fitted using a linear least squares analysis to minimize the difference between the tension data of Katsnelson et al. (11) ( $\left.S_{\text {experiment }}\right)$ and the tension output by the MechChem model $\left(S_{\text {model }}\right)$. The objective function to be minimized is presented in Equation 4.11. The parameter $v_{\max }$ was then fit to the length data of Katsnelson et al.

$$
\text { error }=\frac{\sum_{i=1}^{j}\left(S_{\text {model }}-S_{\text {experiment }}\right)^{2}}{j}
$$




\section{Simulation protocol}

Simulations were performed to mimic isotonic twitch experiments. Given the fitted parameter values, we evaluated how well the model reproduces length and tension data as a function of time in comparison to the isotonic twitch experiments of Katsnelson et al (11). The sarcomere was allowed to contract against seven afterloads ranging from $17 \mathrm{mN}$ to $100 \mathrm{mN}$, as was done in the experiments by Katsnelson et al (11). Throughout all simulations, $2.15 \mu \mathrm{m}$ was the initial and maximum $L_{\text {sarc }}$. The MechChem model outputs the myofiber tension and $L_{\text {sarc }}$ for all steps throughout the cycle.

\section{Results}

\section{Isotonic Twitch}

Figure 4.1 displays the comparison between the experimentally measured isotonic twitch data of Katsnelson et al (11) and the MechChem model-generated isotonic twitch results given the fitted parameter values in Table 4.1. Figure 4.1A and 4.1B display the experimentally measured muscle tension and the MechChem modelgenerated myofiber tension at various afterloads, respectively. Figure $4.1 \mathrm{C}$ displays the experimentally measured muscle length corresponding to the tension curves in Figure 4.1A, and Figure 4.1D displays the MechChem model-generated $L_{\text {sarc }}$ curves.

The MechChem model-generated tension curves are qualitatively similar to the experimental data of Katsnelson et al. The peak tension generated in the isometric contraction (afterload $100 \mathrm{mN}$ ) is higher in the experiments. The duration of the shortening phase is similar between the experiments and the simulated data. In the MechChem model-generated results, the duration of the shortening phase decreased from 0.21 seconds at the lowest afterload to 0.095 seconds at an afterload of $79 \mathrm{mN}$. In the experimental data of Katsnelson et al, the duration of shortening showed a similar decrease in the duration of shortening from 0.23 seconds to 0.12 seconds for the same afterloads. 


\section{Experimental data}

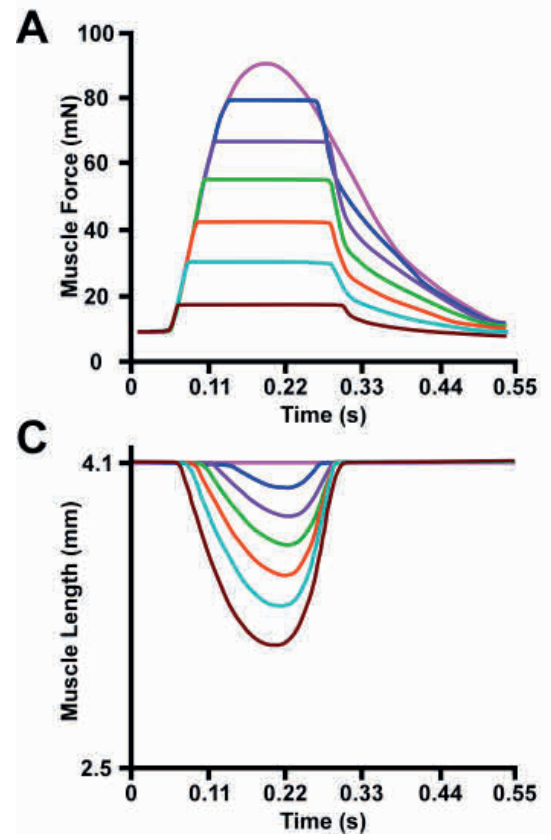

\section{MechChem Results}
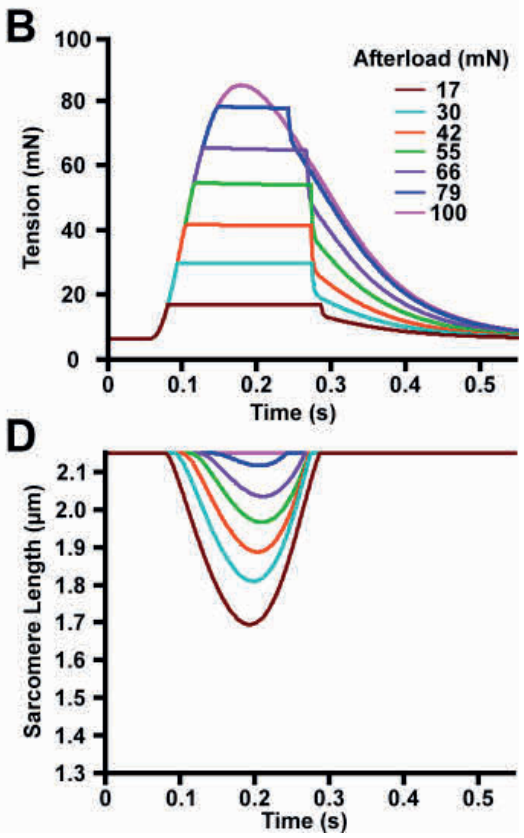

Figure 4.1: Myofiber tension and length in isotonic twitches with seven different maximum afterloads. The (A) muscle force generated in the experimental results of Katsnelson et al. (11) are compared to (B) MechChem model-generated myofiber tension. (C) experimentally measured muscle length is compared to (D) simulated $L_{\text {sarc }}$.

Table 4.1. Fitted parameter values.

\begin{tabular}{lll}
\hline Parameter & Value & Units \\
\hline$C_{s}$ & 0.02 & $\mathrm{kPa}^{-1}$ \\
$a$ & 3.25 & $\mu \mathrm{M} \mathrm{\mu m} \mathrm{m}^{-1}$ \\
$b$ & 8.56 & $\mu \mathrm{M}$ \\
$F_{\text {iso }}$ & 0.29 & $\mathrm{kPa} \mathrm{nm}^{-1}$ \\
$v_{\max }$ & 5.77 & $\mu \mathrm{m} \mathrm{s}^{-1}$ \\
\hline
\end{tabular}




\section{Discussion and conclusions}

In this study, we have expanded the MechChem model of mechano-chemical interactions in cardiac sarcomere contraction to simulate an isotonic twitch. The sarcomere was allowed to shorten against various afterloads, and the model-predicted myofiber tension and length. The model-generated results were in qualitative agreement with the experimental results of Katsnelson et al. (11).

In the experimental data of Katsnelson et al, the tension traces show that the fully isometric twitch requires the longest time to relax. It has been proposed that greater tension developed in the sarcomere causes a longer duration of contraction (4). We propose that the prolongation of contraction is due to the tension dependent mechanism of cooperativity in sarcomere contraction. Greater developed tension in the thin filament hinders relaxation. In this study, the maximum tension was limited by afterload. In experiments, the only shortening phase to peak out past the isometric twitch was that of $79 \mathrm{mN}$. In our simulations, the contractions with an afterload of 79 $\mathrm{mN}$ and $66 \mathrm{mN}$ peaked out past the isometric tension trace. As maximum tension was decreased, the duration of relaxation also decreased.

A greater single overlap length has been shown to allow greater tension to develop in the thin filament (12). Shortening of sarcomeres results in smaller SORs. If the afterload is low, the sarcomere shortens to a smaller length, decreasing the length where a strongly bound $X B$ can develop. Hence, the overall force density decreases. This could help explain the reduced duration of the isotonic twitch at lower afterloads. When $\mathrm{Ca}^{2+}$ is decreased in the cytosol, low tension in the thin filament, partially due to the short SOR, does not have a large hindering effect on relaxation.

The MechChem model-generated relaxation phase begins quickly following the end of the shortening phase. As $L_{\text {sarc }}$ increases, a slower phase of relaxation begins. With a longer SOR, there are greater probabilities of strong $X B S$ developing, slowing the relaxation phase. 
We compare the $L_{\text {sarc }}$ generated by the MechChem model to left ventricular papillary muscle length output in the experiments of Katsnelson et al (11). While muscle length and $L_{\text {sarc }}$ are not directly comparable, the general trends in length change remain similar. The shortening phase is slower than the relengthening phase in both experimental data and MechChem model results.

The MechChem model utilizes a prescribed $\mathrm{Ca}^{2+}$ transient as input. It has been shown that the free intracellular $\mathrm{Ca}^{2+}$ transient is affected by the feedback of force on the $\mathrm{Ca}^{2+}$ handling system (13). However, the $\mathrm{Ca}^{2+}$ transient was not measured in the experiments of Katsnelson et al (11), so these effects could not be viewed in the experimental data. In the future, however, the MechChem model can be coupled to a $\mathrm{Ca}^{2+}$ handling system to understand changes in the calcium transient due to the feedback of mechanics during sarcomere shortening as well as during the relaxation phase.

The addition of the ability for the sarcomere to shorten is a necessary step added to the MechChem model. In a whole heart, sarcomeres shorten during systole, and blood is ejected from the left ventricle. Hence, for the MechChem model to be utilized within the framework of a full heart contraction model, sarcomere shortening must be possible. With this development, it is a logical next step to insert the MechChem model into the framework of a whole-heart model such as the CircAdapt model of the closed-loop circulation.

The MechChem model has been extended to allow the sarcomere to change length. The model-generated tension and $L_{\text {sarc }}$ curves during isotonic twitches at various afterloads are in good qualitative agreement with experimental results. This development step in the MechChem model will allow its use to couple it with a calcium handling system as well as a whole-heart model. 


\section{References}

1. Solaro RJ, Rarick HM. Troponin and Tropomyosin: Proteins That Switch on and Tune in the Activity of Cardiac Myofilaments. Circulation Research. 1998;83(5):471-80.

2. Dobesh DP, Konhilas JP, de Tombe PP. Cooperative activation in cardiac muscle: impact of sarcomere length. American Journal of Physiology - Heart and Circulatory Physiology. 2002;282(3):H1055H62.

3. Dupuis LJ, Lumens J, Arts T, Delhaas T. Mechano-chemical Interactions in Cardiac Sarcomere Contraction: A Computational Modeling Study. PLOS Computational Biology. 2016;12(10):e1005126.

4. Janssen PM, Hunter WC. Force, not sarcomere length, correlates with prolongation of isosarcometric contraction. American Journal of Physiology. 1995;269(2):H676-H85.

5. Kassiri Z, Myers R, Kaprielian R, Banijamali HS, Backx PH. Rate-dependent changes of twitch force duration in rat cardiac trabeculae: a property of the contractile system. The Journal of Physiology. 2000;524(Pt 1):221-31.

6. Sun Y-B, Lou F, Irving M. Calcium- and myosin-dependent changes in troponin structure during activation of heart muscle. The Journal of Physiology. 2009;587(Pt 1):155-63.

7. Hill AV. The Heat of Shortening and the Dynamic Constants of Muscle. 1938;126(843):136-95.

8. Rice JJ, Wang F, Bers DM, de Tombe PP. Approximate Model of Cooperative Activation and Crossbridge Cycling in Cardiac Muscle Using Ordinary Differential Equations. Biophysical Journal. 2008;95(5):2368-90.

9. Landesberg A, Sideman S. Mechanical regulation of cardiac muscle by coupling calcium kinetics with cross-bridge cycling: a dynamic model. American Journal of Physiology. 1994;267(2):H779-H95.

10. Weiwad WKK, Linke WA, Wussling MHP. Sarcomere Length-tension Relationship of Rat Cardiac Myocytes at Lengths Greater than Optimum. Journal of Molecular and Cellular Cardiology. 2000;32(2):24759.

11. Katsnelson LB, Nikitina LV, Chemla D, Solovyova O, Coirault C, Lecarpentier Y, et al. Influence of viscosity on myocardium mechanical activity: a mathematical model. Journal of Theoretical Biology. 2004;230(3):385-405.

12. Gordon AM, Homsher E, Regnier M. Regulation of Contraction in Striated Muscle. Physiological Reviews. 2000;80(2):853-924.

13. Kurihara S, Komukai K. Tension-dependent changes of the intracellular Ca2+ transients in ferret ventricular muscles. The Journal of Physiology. 1995;489(Pt 3):617-25. 




\section{Chapter 5}

\section{Linking cross-bridge cycling kinetics to}

\section{response to cardiac resynchronization therapy:}

\section{A Multiscale modeling study}

This chapter is based on:

Lauren J. Dupuis, Joost Lumens, Theo Arts, and Tammo Delhaas (2018). Linking crossbridge cycling kinetics to response to cardiac resynchronization therapy: A Multiscale modeling study. In Europace. (Accepted).

\section{Abstract}

Aims: Cardiac resynchronization therapy (CRT) is currently the most widely used treatment for heart failure patients with left bundle branch block (LBBB). In recent years, the presence of septal rebound stretch has been found to be a positive indicator for CRT response although the mechanism is unknown. Methods: In an attempt to understand the relation between cellular mechanics and global pump function in CRT patients, we utilize the CircAdapt closed-loop cardiovascular system model in combination with the MechChem model of cardiac sarcomere contraction. LBBB has been simulated with increasing delay in left ventricular (LV) free wall and septal wall activation. In addition to the electrical dyssynchrony, myocardial mechanical function was diminished by decreasing the cross-bridge cycling rate. Results: Our results have shown that a decrease in the cross-bridge cycling rate in addition to LBBB resulted in a decrease in septal rebound stretch (SRS) with a concomitant decreased response to 
resynchronization. Conclusions: The results of our multiscale modeling study suggest that, while greater SRS during systole clearly indicates electrical dyssynchrony, it also predicts mechanical viability and healthy cross-bridge cycling rates in the myocardium. Hence, septal rebound stretch positively indicates response to CRT.

\section{Introduction}

Cardiac resynchronization therapy (CRT) is currently the most widely used treatment option for chronic heart failure (HF) patients with left bundle-branch block (LBBB) and reduced left ventricular ejection fraction $($ LVEF) $\leq 35 \%$ (1). However, many patients that meet all clinical criteria for CRT do not respond to this treatment for unknown reasons (2). It has been hypothesized that some patients with an electrical delay are less responsive to electrical resynchronization because they suffer from a mechanical deficiency in the myocardial tissue (3).

It has been shown previously that septal strain patterns can aid in the determination of prognosis of HF patients with LBBB (4); a baseline septal strain pattern featuring early-systolic shortening followed by rebound stretch during the ejection phase has been shown to be a signature of good response to CRT. In contrast, LBBB patients with a septal strain pattern showing a late-systolic shortening peak and little or no systolic rebound stretch were associated with less favorable response to CRT, in terms of LV reverse remodeling. These results have been reproduced by other investigators who also showed that patients with little or no septal systolic rebound stretch have a significantly worse clinical outcome than patients with septal systolic rebound stretch either or not in combination with systolic pre-stretch of the postero-lateral wall $(3,5)$. The worse outcome related to lower septal rebound stretch was attributed to a decrease in contractility of the tissue. However, the exact mechanisms underlying on the one hand the absence of septal systolic rebound stretch despite a clear electrocardiographic LBBB morphology and on the other hand the relation between absent systolic rebound stretch and non-response to CRT remain unclear. 
The sarcomeric cross-bridge cycle is fundamental to the mechanical force development in cardiac muscle (6). An experimental study by Alpert et al. (7) suggested that the maximum velocity of sarcomere shortening, which is among others determined by the cross-bridge cycling rate, decreases with age as well as in heart failure. This could be due to either stiffening of tissue or an increase in the time crossbridges remain attached. Additionally, ATP is necessary to detach cross-bridges. The creatine kinase reaction resynthesizes ATP when the heart is under increased load. It has been shown that the creatine kinase reaction decreases in function during heart failure (8).

In this study, we hypothesize that decreased sarcomere shortening velocity can explain both the absence of septal systolic rebound stretch in LBBB patients and the limited functional improvement they gain from CRT. We utilize the CircAdapt closedloop cardiovascular system model (9) in combination with the MechChem model of cardiac sarcomere contraction (10) to better understand the relation between cellular mechanics and global pump function.

\section{Methods}

The CircAdapt model of the closed-loop cardiovascular system was used to investigate effects of dyssynchronous activation and decreased cross-bridge cycling on hemodynamics and septal strain patterns. The CircAdapt model allows real-time beatto-beat simulation of cardiac mechanics and circulatory hemodynamics (9). Modules that represent sarcomere mechanics, myocardial walls, valves, the pericardium, large blood vessels, and peripheral resistances are included in the CircAdapt model. The TriSeg model (11) is utilized to mechanically couple the three ventricular walls using the concept of conservation of energy. Within each wall, the stress is uniform, so it can be calculated as a single muscle fiber (12). Prior to the current study, the CircAdapt model utilized a phenomenological model of sarcomere mechanics as described in detail by Lumens et al. (11) in which the sarcomere was represented by a three element Hill model that prescribes an increase in force development and duration of contraction for greater sarcomere lengths. Calcium activation was not explicitly 
accounted for, but was instead incorporated in a contractility curve formulation. For this study, the phenomenological module representing sarcomere mechanics has been replaced in the CircAdapt modeling platform by the more physics- and physiologybased MechChem model (10) describing the mechano-chemical interactions underlying cardiac sarcomere contraction.

The MechChem model, shown schematically in Figure 5.1, takes activation time as input and outputs the tension developed in the sarcomere. Sarcomere tension is calculated for each time $(t)$ and position along the single overlap region of the thin filament $(x)$. The MechChem model utilizes a two-state thin filament activation model: the non-permissive state $N(x, t)$ where the binding sites for cross-bridges are blocked, and the permissive state $P(x, t)$ where calcium is bound to the troponin complex and the binding sites are unblocked for cross-bridges to bind. The cross-bridge cycle model consists of a detached, non-force-generating state $D(x, t)$, and an attached, forcegenerating state $A(x, t) . A(x, t)$ determines a cross-bridge density locally along the thin filament, and it determines the tension in the thin filament. High tension in the thin filament locally blocks the transition of the thin filament from the permissive state to the non-permissive state. The CircAdapt model determines the initial length of the sarcomere and the time the tissue is activated, triggering the calcium transient and thus sarcomeric force generation. The MechChem model outputs the active myofiber stress. 


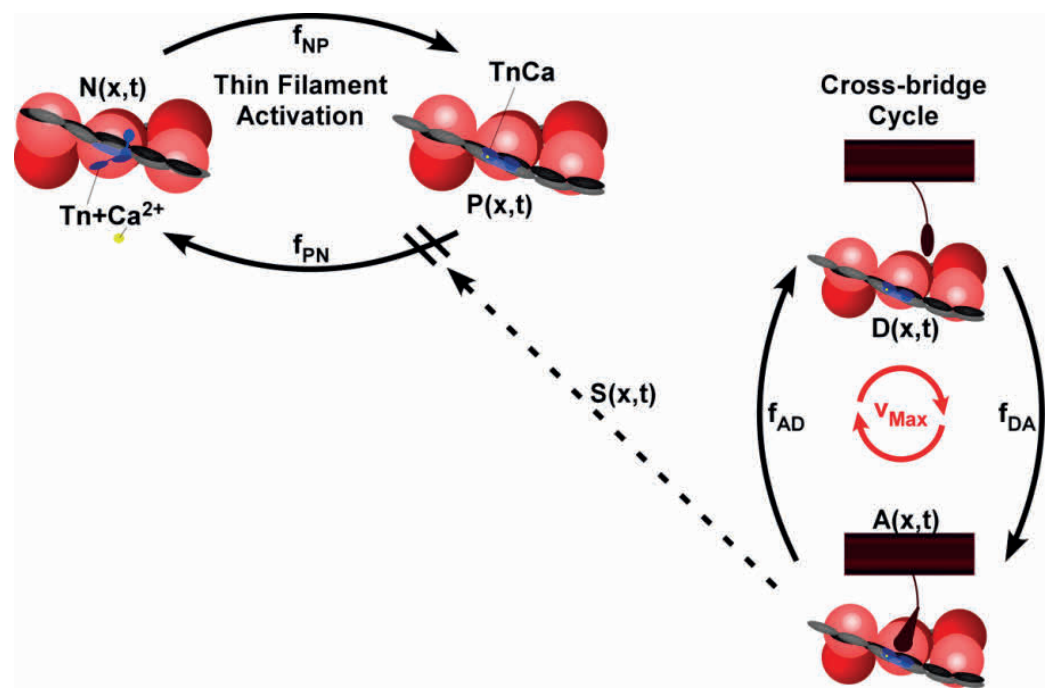

Figure 5.1: MechChem model schematic. The MechChem model of mechano-chemical interactions in cardiac sarcomere contraction consists of two separate processes, the binding of calcium ions $\left(\mathrm{Ca}^{2+}\right)$ to troponin ( $\mathrm{Tn}$ ) to activate the cardiac muscle, and the cross-bridge cycle. The thin filament is initially at rest, so the binding sites for cross-bridges are blocked, thus forming the non-permissive thin filament state $(\mathrm{N}(\mathrm{x}, \mathrm{t})) . \mathrm{Ca}^{2+}$ can bind to Tn, triggering conformational changes that result in the unblocking of the crossbridge sites, changing the thin filament to the permissive state $(P(x, t))$. Initially, cross-bridges are not bound $(D(x, t))$. A cross-bridge can bind to the thin filament in the attached, force generating state of the crossbridge $(A(x, t))$. The maximum velocity of shortening $\left(v_{M a x}\right)$ is determined by the cross-bridge cycling rate. The tension in the thin filament determined by the cross-bridges in state $A(x, t)$ blocks the transition of the thin filament from state $P(x, t)$ to $N(x, t)$.

\section{Simulation Protocol}

The following simulations were performed: a reference simulation with synchronous activation of the ventricular wall, LBBB simulation with various degrees of asynchrony, and LBBB with mechanic dysfunction. During all simulations, mean arterial pressure was maintained at $92 \mathrm{mmHg}$ while cardiac output was maintained at $3.8 \mathrm{~L} / \mathrm{min}$ to mimic heart failure (13). To this end we applied homeostatic control, featuring alterations of circulating blood volume and systemic vascular resistance. Heart rate was assumed constant at $70 \mathrm{bpm}$ in all simulations. An LBBB-like activation pattern was simulated by delaying the activation from the right ventricular wall to the LV free wall by $25 \mathrm{~ms}$ to represent a mild LBBB up to $75 \mathrm{~ms}$ to represent a severe LBBB. The 
septal wall was activated at one-third of the delay between the RV free wall and the LV free wall. In the CircAdapt model, the delay between LV and RV free wall activation is not equivalent to the QRS duration. The activation times refer to the average activation time of all of the tissue in the wall. Hence, the delay used in model simulations is not equal to the delay between the earliest activated RV tissue and latest activated LV tissue but the delay between the average activation time of all of the RV and LV tissue. A 50 ms delay in activation from the RV to the LV free walls was utilized for all simulations to match an average CRT candidate, based on human measurements by Huntjens et al (14), unless otherwise specified. The parameter determining the maximum shortening velocity of the sarcomere, $v_{M a x}$, was gradually decreased from $100 \%$ to $40 \%$. Previous studies have related a late peak septal strain pattern in LBBB to the weakening of myocardial tissue (4). In our study, $v_{\operatorname{Max}}$ was reduced to $40 \%$ of its value because it led to the clear late peak septal strain pattern that has been described.

To assess the function of the heart, LV ejection fraction (EF) and end-diastolic pressure (EDP) were calculated. To assess the distribution of regional LV tissue load, the work performed by the LV free wall tissue and the septal wall tissue were calculated as the integral of the simulated myofiber stress-natural strain relationship in those tissues multiplied with the wall volume. The estimated onset QRS was determined by the average onset of ventricular calcium transients. This point was used as the zero-strain reference point.

\section{Results}

Figure 5.2 shows the effects of increased dyssynchrony on cardiac function. The delay in LV free wall activation time increases from left to right. Pressure-volume loops, engineering strain, and myofiber stress-strain loops are displayed in Panels $A, B$, and $C$, respectively. The results in Panel A show the progressive dilation of the LV with enddiastolic volumes increasing from $100 \mathrm{~mL}$ during synchronous contraction to $129 \mathrm{~mL}$ when the LV free wall activation is delayed by $50 \mathrm{~ms}$. The strain patterns shown in Panel B during a synchronous contraction show relatively uniform shortening during 
ejection and relengthening during filling in all three ventricular walls. With increased delay in LV free wall activation, both LV free wall pre-stretch and septal stretch during systole increased. Greater septal stretch during systole resulted in less and even negative work done by the septum.

LV Free wall activation delay

A

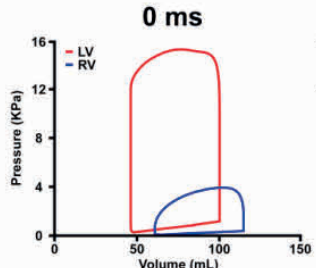

B

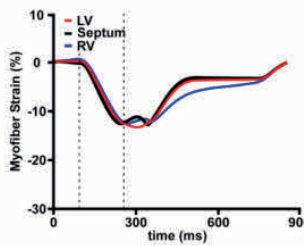

C

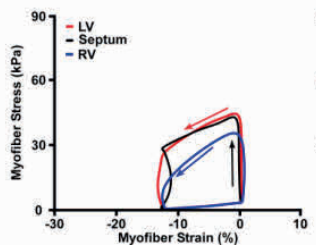

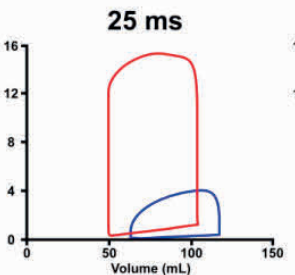
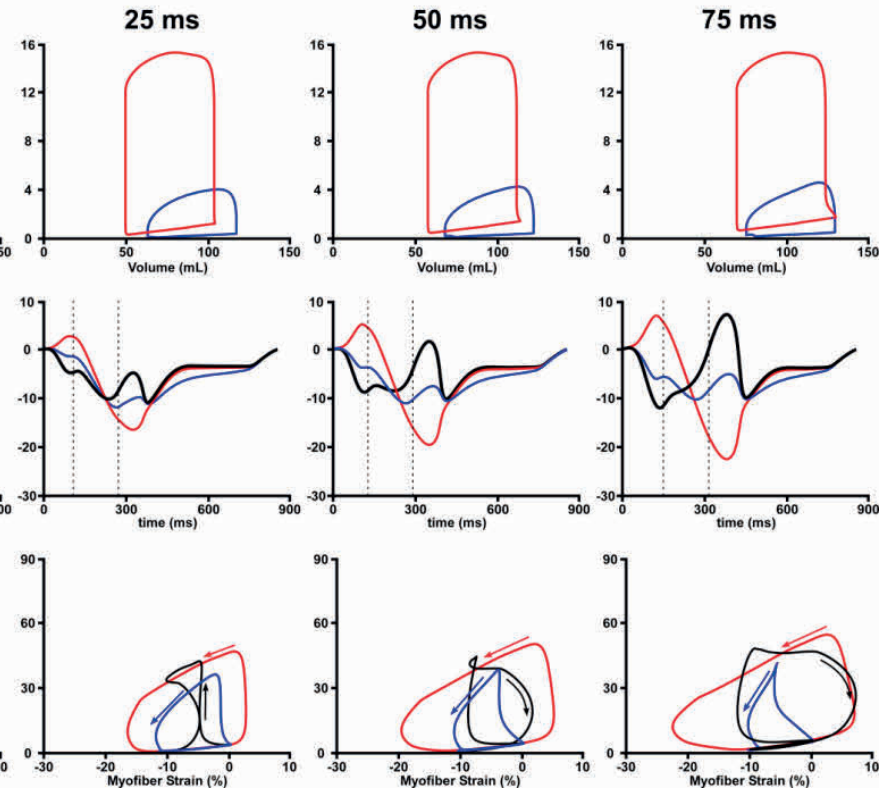

Figure 5.2: Effects of increased dyssynchrony on cardiac function in the reference and LBBB simulations. Dyssynchrony increases from left to right $(0 \mathrm{~ms}, 25 \mathrm{~ms}, 50 \mathrm{~ms}, 75 \mathrm{~ms})$. The resulting (A) pressure-volume loops in the right ventricle (blue) and left ventricle (red) are displayed. (B) Engineering strain in the right ventricle (blue), left ventricle (red), and septum (black) are shown. The vertical dotted lines denote the ejection phase.(C) Myofiber stress-strain loops in left ventricular tissue (red), right ventricular tissue (blue), and septal tissue (black) are shown. Arrows indicate the direction of the loops.

Figure 5.3A displays the septal strain pattern resulting from an LBBB with a $50 \mathrm{~ms}$ delay in LV free wall activation and a $16.7 \mathrm{~ms}$ delay in septal wall activation. The septum exhibits clear septal rebound stretch (red segments). Figure 5.3B displays the decrease in septal rebound stretch with decreasing $\mathrm{v}_{\mathrm{Max}}$. The septal rebound stretch decreases from $5.6 \%$ to $1.3 \%$ with decreasing $\mathrm{v}_{\mathrm{Max}}$ from $100 \%$ of its maximal value to $40 \%$ of its maximal value. 

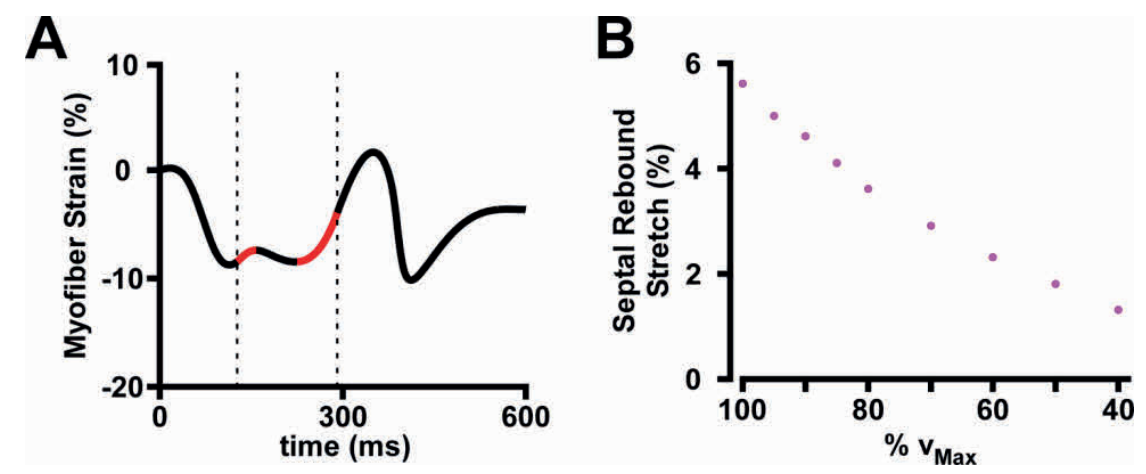

Figure 5.3: Septal rebound stretch in an LBBB heart with a 50 ms delay in LV free wall activation. (A) The septal rebound stretch is illustrated in the red segment of the septal strain pattern. (B) Changes in septal rebound stretch with decreasing $\mathrm{v}_{\mathrm{Max}}$ down to $40 \%$ of its maximal value are displayed.

\section{Global Pump Function}
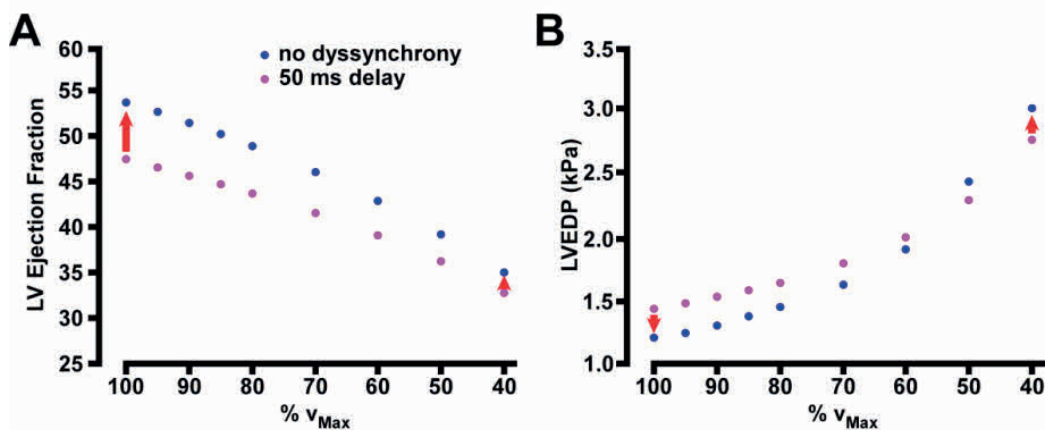

Regional Tissue Mechanics
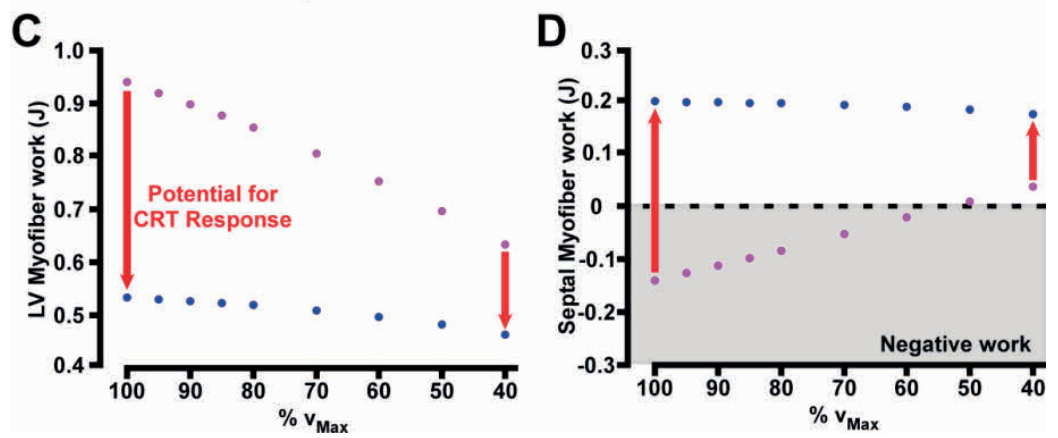

Figure 5.4: Changes in pump function with no dyssynchrony (blue) and 50 ms delay in LV free wall activation (magenta) with decreasing $\mathbf{v}_{\mathbf{M a x}}$. The changes shown are $(A)$ the LV ejection fraction, (B) LV End Diastolic Pressure (EDP), (C) LV myofiber work, and (D) the septal myofiber work. Red arrows denote the potential for CRT response. 
Figure 5.4 displays the changes in global pump function with decreasing maximal sarcomere shortening velocity $\left(\mathrm{v}_{\mathrm{Max}}\right)$ during synchronous activation (blue dots) and with LBBB corresponding to $50 \mathrm{~ms}$ delay in LV free wall activation (magenta dots). A $60 \%$ decrease in $\mathrm{V}_{\mathrm{Max}}$ in a synchronous failing heart resulted in decreased LV ejection fraction from $54 \%$ to $35 \%$, an increase in LVEDP from 1.2 to $3.0 \mathrm{KPa}$, a decrease in LV myofiber work from $0.53 \mathrm{~J}$ to $0 .-46 \mathrm{~J}$, and a decrease in septal myofiber work from 0 . $20 \mathrm{~J}$ to $0.17 \mathrm{~J}$. The same decrease in $\mathrm{v}_{\mathrm{Max}}$ in LBBB simulations with a $50 \mathrm{~ms}$ delay resulted in a decrease in LV ejection fraction from $47 \%$ to $33 \%$, an increase in LVEDP from 1.4 to $2.8 \mathrm{KPa}$, a decrease in LV myofiber work from $0.94 \mathrm{~J}$ to $0.63 \mathrm{~J}$, and an increase in septal myofiber work from $-0.14 \mathrm{~J}$ to $0.04 \mathrm{~J}$. Additionally, the relative difference in LVEF between healthy and LBBB hearts decreased with decreasing $v_{\text {Max }}$.

Figure 5.5 displays the comparison between simulated septal strain patterns (top row) and clinically measured septal strain patterns of a healthy volunteer and two patients with LBBB (bottom row). The reference simulation is shown in Figure 5.5A, whereas LBBB simulations with a 50 ms delay in LV free wall activation is shown in Figure 5.5B. Figure 5.5C displays simulated strain patterns in LBBB with 50 ms delay in free wall activation time in combination with a $60 \%$ decrease in $\mathrm{v}_{\text {Max }}$. The normal control is shown in Figure 5.5D, an LBBB septal strain pattern with septal rebound stretch is shown in Figure 5.5E, and a late peak septal strain patter in LBBB is shown in Figure 5.5F. Qualitatively, simulations compare well to the experimentally measured data. Ejection begins in the dyssynchrony only case (Figure 5.5B and 5.5E) after initial shortening of the septal wall, and the septum begins lengthening at the beginning of ejection in both cases. When $\mathrm{v}_{\mathrm{Max}}$ is decreased to $40 \%$ of its healthy value, the septum is still shortening when the ejection phase begins. There is a similar late peak in septal shortening during ejection in both the simulations and the experimentally measured data (Figure 5.5F). In both cases, the septal wall begins to relengthen before the end of ejection. 

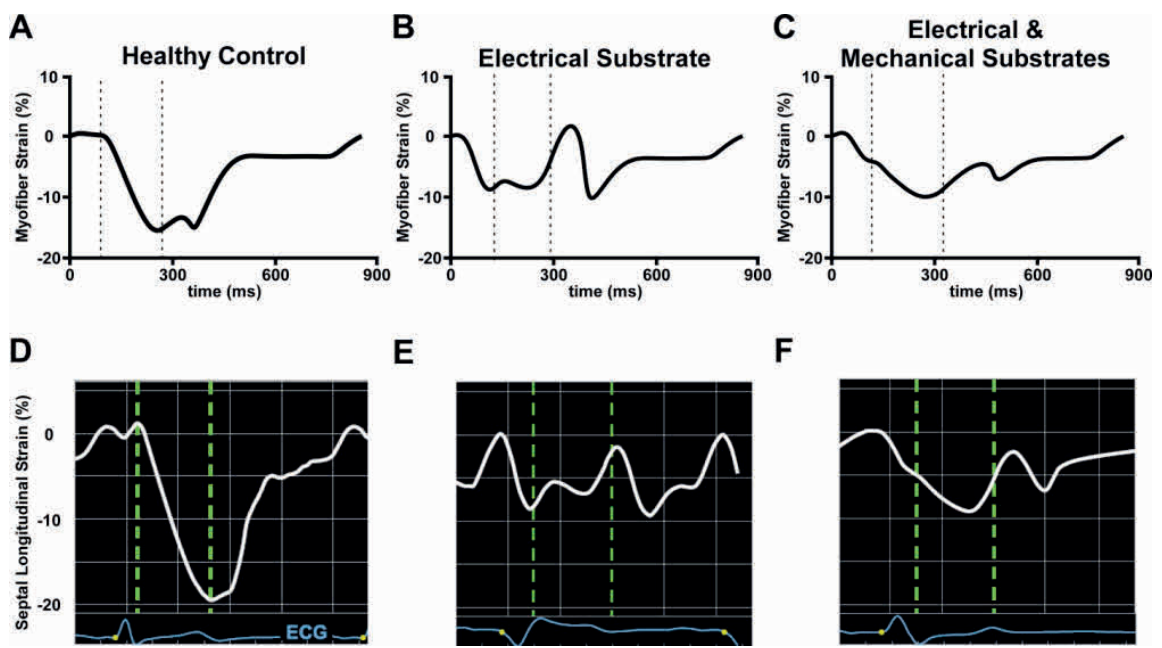

E

F
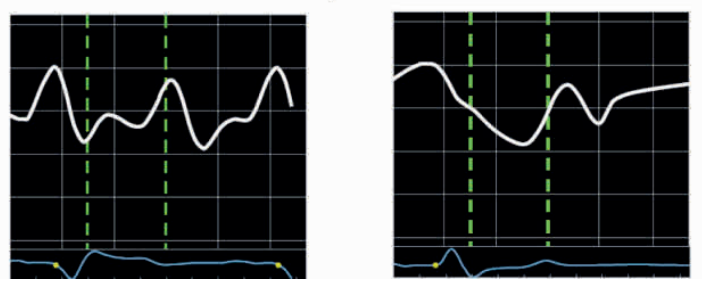

Figure 5.5: Comparison of simulated and experimentally measured septal strain patterns from Leenders et al. (4). Simulated septal myofiber strain patterns are shown on the top (A, B, C), while the bottom row displays septal longitudinal strain patterns measured in a healthy volunteer and two heart failure patients with $\operatorname{LBBB}(D, E, F)$. (A and D) Strain patterns resulting from synchronous contraction are displayed. (B and E) A double-peak septal strain pattern is compared to a simulations with solely an electrical substrate, i.e. a LV free wall activation delay of $50 \mathrm{~ms}$ and a septal wall activation delay of $16.7 \mathrm{~ms}$. (C and F) The late-peak septal strain pattern is compared to a simulation with both an electrical (LV free wall activation delay of 50 $\mathrm{ms}$ ) and a mechanical ( $\mathrm{v}_{\operatorname{Max}}$ is reduced to $40 \%$ ) substrate. The dashed lines indicate LV ejection.
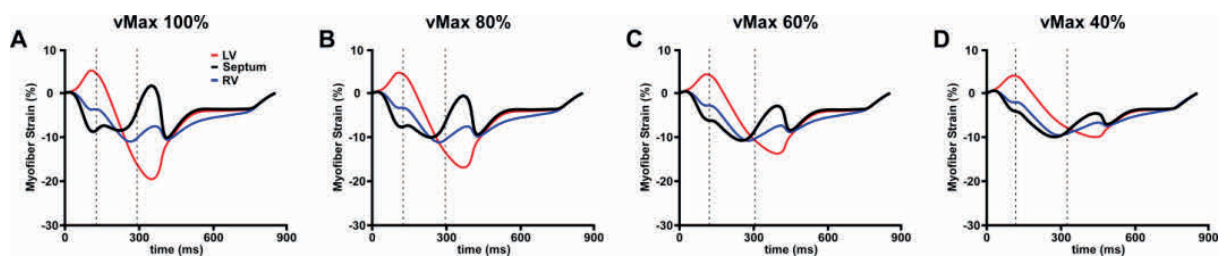

Figure 5.6: Progression of strain patterns with reduction in $\mathbf{v}_{\text {Max }}$ ( $A$ ) The strain patterns in the LV (red), septum (black), and RV (blue) are displayed for the LBBB simulation with the electrical substrate alone (LV free wall activation delay of $50 \mathrm{~ms}$ ). The strain patterns are displayed with a reduction in $\mathrm{v}_{\operatorname{Max}}$ to (B) $80 \%,(C)$ $60 \%$, and (D) $40 \%$ of its healthy value, while the electrical substrate is kept constant. Dotted lines denote LV ejection. 
Figure 5.6 displays the progression of strain patterns in an LBBB heart with gradual reductions in $v_{\operatorname{Max}}$ from $100 \%$ to $40 \%$. As $v_{\text {Max }}$ reduces, the degree of septal rebound stretch diminishes, and the septal wall no longer relengthens early in the ejection phase. In addition to the diminished septal lengthening as $\mathrm{v}_{\mathrm{Max}}$ decreases, the LV free wall shortens less. The relative differences between LV and septal myofiber strain at the peaks decrease, making the strain patterns appear more synchronous in the different tissue segments.

\section{Discussion}

For this study, the MechChem model of cardiac sarcomere contraction was integrated in the CircAdapt model of the closed circulation. The model was used to understand the effects of electrical delay, with or without a decrease in the cross-bridge cycling rate, on regional myocardial deformation and on global cardiac pump function. Our results underlined our hypothesis that decreases in cross-bridge cycling rate diminish septal rebound stretch that, on its turn, signals decreased mechanical viability of the myocardium and poor CRT response.

\section{Relative functional gains from cardiac resynchronization therapy deplete with decreased $\mathbf{v}_{\operatorname{Max}}$}

We manipulated a mechanical property of cardiac tissue, the maximum velocity of sarcomere shortening $\left(\mathrm{v}_{\mathrm{Max}}\right)$, which is depending on the cross-bridge cycling rate. With decreases in $\mathrm{V}_{\mathrm{Max}}$ in both a healthy heart and an LBBB heart, LVEF decreased. Additionally, both the absolute and relative difference in LVEF between the healthy and $L B B B$ case decreased with decreasing $\mathrm{V}_{\mathrm{Max}}$, suggesting that the potential for functional improvement by CRT decreases. We predict increases in LVEF with CRT treatment of 6.3 and $2.3 \%$-points with $\mathrm{v}_{\mathrm{Max}}$ at $100 \%$ and $40 \%$, respectively. Mechanical function of the tissue is poor when $\mathrm{v}_{\mathrm{Max}}$ is low, so the $2.3 \%$ points gain induced by CRT only increases LVEF to $35 \%$. An LVEF of $35 \%$ is considered to be a reduced EF (15), so treatment with CRT would not return the EF to a normal range. Simulation results showed that LVEDP increased with decreasing $v_{\operatorname{Max}}$ in both the LBBB simulation and the synchronous simulation. Like LVEF, the relative differences between LVEDP of the 
synchronous and LBBB hearts decreased with decreasing $\mathrm{v}_{\text {Max }}$. However, when $\mathrm{v}_{\operatorname{Max}}$ was decreased past $60 \%$ of its healthy value, LVEDP was higher in synchronously activated hearts than in the ones with LBBB.

Simulation results showed that as $\mathrm{v}_{\mathrm{Max}}$ decreases, ventricular strain patterns become more uniform during ejection. In LBBB hearts with a decrease in $\mathrm{v}_{\operatorname{Max}}$ to $40 \%$ of its maximum value, the ventricular walls appear to contract nearly synchronously. The decrease in cross-bridge cycling rate results in less shortening of the tissue in the LV free wall. Hence, the LV free wall cannot stretch the septal tissue to the degree it would if the cross-bridge cycling rate was higher. Additionally, LV free wall pre-stretch decreases with decreasing $\mathrm{v}_{\mathrm{Max}}$, resulting in a less forceful contraction of the LV free wall. The decreased cross-bridge cycling rate also corresponds to the homogenization of ventricular tissue strain rates during ejection. Our results suggest that in the failing heart a relatively normal cross-bridge cycling rate is essential for 1) septal systolic rebound stretch and pre-stretch of the LV free wall in LBBB patients and also for 2) improvement of LV systolic pump function after electrical resynchronization. These findings may explain why systolic stretch (i.e. the sum of LV free wall pre-stretch and septal systolic rebound stretch) has been shown to identify patients who benefit more favorably from CRT, including those with intermediate electrocardiographic criteria (i.e., QRS duration of 120-149 ms or non-LBBB), where CRT response is less certain by ECG alone (3).

\section{Myofiber work distribution with decreasing $\mathbf{v}_{\mathrm{Max}}$}

In our simulations, $\mathrm{v}_{\mathrm{Max}}$ appears to have little impact on myofiber work in the LV free wall and septum when the heart contracts synchronously. Our results show that a $60 \%$ decrease in $\mathrm{v}_{\mathrm{Max}}$ in a synchronous failing heart decreases LV and septal myofiber work by $7.1 \%$ and $2.4 \%$, respectively. The addition of electrical dyssynchrony, however, results in a complete redistribution of work.

It is documented that the septum performs negative work in severe cases of LBBB due to the stretch in septal tissue during systole (16-18). In our simulations, the negative 
work performed by the septum increases with dyssynchrony. Our results showed that a $60 \%$ decrease in $\mathrm{v}_{\mathrm{Max}}$ from its maximum value caused a $33 \%$ decrease in LV free wall myofiber work and completely diminished the negative work of the septum. It has been proposed that negative work in the septum can be saved and turned into positive work with CRT (18). Our simulations predict that nearly all of the negative septal work can be turned to positive work with CRT when $\mathrm{v}_{\mathrm{Max}}$ is $100 \%$. In simulations where $\mathrm{v}_{\mathrm{Max}}$ is reduced to $40 \%$, the septum no longer performs negative work because the LV free wall is not able to stretch it as much during systole. Hence, the potential for an increase in septal work by CRT is less. Our results show that negative septal work resulting from septal rebound stretch is a positive indicator for CRT response. Besides the presence of an LBBB electrical activation pattern, it indicates that the tissue is still mechanically viable. When the strain patterns appear relatively synchronous and the septum exhibits the late peak pattern in systole, the tissue has worse mechanical deficiency and has less to gain from CRT.

\section{Limitations}

In this study, we utilize a model with a simplified geometry. The LV, septum, and RV are each represented by a single, lumped contraction model (11). Hence, it is not possible to represent tissue inhomogeneities within a wall. However, this study was chosen to focus on global changes in mechanical parameters, so the lumped models were suitable. It is currently impossible to measure $\mathrm{v}_{\operatorname{Max}}$ in tissue in the human heart in vivo, so changes in this parameter cannot be compared to experimental data. Additionally, Kirk and Kass (19) summarized their previously published experimental data in a recent review and pointed out that heart failure combined with LBBB can induce alterations at the sub-cellular level that can impact whole-heart function. The alterations include changes to the intracellular calcium transient, the sensitivity of the thin filament proteins to calcium, the flow of ions across specific channels, and cellular metabolic remodeling, among other possible changes. These possible sub-cellular changes, while outside the scope of the current study, present possible future research. 


\section{Conclusions}

A subset of patients with heart failure and left bundle branch block (LBBB) meet all current clinical criteria to receive cardiac resynchronization therapy (CRT), yet respond negatively to treatment for unknown reasons. Our study has shown that a decreased cross-bridge cycling rate in cardiac tissue, in addition to an electrical delay, results in a change in septal strain pattern morphology. Ventricular tissues shorten more homogeneously during systole in LBBB with decreased cross-bridge cycling rates, producing a late peak septal strain pattern that is a predictor of poor CRT response. We have shown that the slowed cross-bridge cycling rate reduces possible gains in pump function from electrical resynchronization. Conversely, patients exhibiting a greater degree of septal rebound stretch during systole tend to positively respond to CRT. The results of our study suggest that septal rebound stretch predicts healthy mechanical function in cardiac tissue and positive response to CRT. 


\section{References}

1. Brignole M, Auricchio A, Baron-Esquivias G, Bordachar P, Boriani G, Breithardt O-A, et al. 2013 ESC Guidelines on cardiac pacing and cardiac resynchronization therapyThe Task Force on cardiac pacing and resynchronization therapy of the European Society of Cardiology (ESC). Developed in collaboration with the European Heart Rhythm Association (EHRA). EP Europace. 2013;15(8):1070-118.

2. Leyva F, Nisam S, Auricchio A. 20 Years of Cardiac Resynchronization Therapy. Journal of the American College of Cardiology. 2014;64(10):1047-58.

3. Lumens J, Tayal B, Walmsley J, Delgado-Montero A, Huntjens PR, Schwartzman D, et al. Differentiating Electromechanical From Non-Electrical Substrates of Mechanical Discoordination to Identify Responders to Cardiac Resynchronization Therapy. Circulation: Cardiovascular Imaging. 2015;8(9).

4. Leenders GE, Lumens J, Cramer MJ, De Boeck BWL, Doevendans PA, Delhaas T, et al. Septal Deformation Patterns Delineate Mechanical Dyssynchrony and Regional Differences in Contractility: Analysis of Patient Data Using a Computer Model. Circulation Heart Failure. 2012;5(1):87-96.

5. Maréchaux S, Guiot A, Castel AL, Guyomar Y, Semichon M, Delelis F, et al. Relationship between Two-Dimensional Speckle-Tracking Septal Strain and Response to Cardiac Resynchronization Therapy in Patients with Left Ventricular Dysfunction and Left Bundle Branch Block: A Prospective Pilot Study. Journal of the American Society of Echocardiography. 2014;27(5):501-11.

6. Gordon AM, Homsher E, Regnier M. Regulation of Contraction in Striated Muscle. Physiological Reviews. 2000;80(2):853-924.

7. Alpert NR, Mulieri LA, Warshaw D. The Failing Human Heart. Cardiovascular Research. 2002;54:110.

8. Liao R, Nascimben L, Friedrich J, Gwathmey JK, Ingwall JS. Decreased Energy Reserve in an Animal Model of Dilated Cardiomyopathy. Relationship to Contractile Performance. 1996;78(5):893-902.

9. Arts T, Delhaas T, Bovendeerd P, Verbeek X, Prinzen FW. Adaptation to mechanical load determines shape and properties of heart and circulation: the CircAdapt model. American Journal of Physiology - Heart and Circulatory Physiology. 2005;288(4):H1943-H54.

10. Dupuis LJ, Lumens J, Arts T, Delhaas T. Mechano-chemical Interactions in Cardiac Sarcomere Contraction: A Computational Modeling Study. PLOS Computational Biology. 2016;12(10):e1005126.

11. Lumens J, Delhaas T, Kirn B, Arts T. Three-Wall Segment (TriSeg) Model Describing Mechanics and Hemodynamics of Ventricular Interaction. Annals of Biomedical Engineering. 2009;37(11):2234-55.

12. Arts T, Bovendeerd PH, Prinzen FW, Reneman RS. Relation between left ventricular cavity pressure and volume and systolic fiber stress and strain in the wall. Biophysical Journal. 1991;59(1):93-102.

13. Vidal B, Sitges M, Marigliano A, Delgado V, Díaz-Infante E, Azqueta $M$, et al. Optimizing the Programation of Cardiac Resynchronization Therapy Devices in Patients With Heart Failure and Left Bundle Branch Block. The American Journal of Cardiology. 2007;100(6):1002-6. 
14. Huntjens PR, Ploux S, Strik M, Walmsley J, Ritter P, Haissaguerre M, et al. Electrical Substrates Driving Response to Cardiac Resynchronization Therapy. A Combined Clinical-Computational Evaluation. $2018 ; 11(4)$.

15. Ponikowski P, Voors AA, Anker SD, Bueno H, Cleland JGF, Coats AJS, et al. 2016 ESC Guidelines for the diagnosis and treatment of acute and chronic heart failureThe Task Force for the diagnosis and treatment of acute and chronic heart failure of the European Society of Cardiology (ESC)Developed with the special contribution of the Heart Failure Association (HFA) of the ESC. European Heart Journal. 2016;37(27):2129-200.

16. Ghani A, Delnoy PPHM, Adiyaman A, Ottervanger JP, Ramdat Misier AR, Smit JJJ, et al. Septal rebound stretch as predictor of echocardiographic response to cardiac resynchronization therapy. International Journal of Cardiology Heart \& Vasculature. 2015;7:22-7.

17. Russell K, Eriksen M, Aaberge L, Wilhelmsen N, Skulstad H, Gjesdal O, et al. Assessment of wasted myocardial work: a novel method to quantify energy loss due to uncoordinated left ventricular contractions. American Journal of Physiology-Heart and Circulatory Physiology. 2013;305(7):H996-H1003.

18. Vecera J, Penicka M, Eriksen M, Russell K, Bartunek J, Vanderheyden M, et al. Wasted septal work in left ventricular dyssynchrony: a novel principle to predict response to cardiac resynchronization therapy. European Heart Journal - Cardiovascular Imaging. 2016;17(6):624-32.

19. Kirk JA, Kass DA. Cellular and Molecular Aspects of Dyssynchrony and Resynchronization. Cardiac electrophysiology clinics. 2015;7(4):585-97. 




\section{Chapter 6}

\section{General Discussion}

\section{Introduction}

In this thesis we have constructed a novel hypothesis to explain the mechanism of cooperativity in the cardiac sarcomere. The central hypothesis for this work is that an intrinsic chemical cooperativity in the sarcomere is boosted by tension locally along the thin filament. Each force-generating cross-bridge increases the tension along the thin filament from the mid-line toward the z-disk. We propose that high tension tightens the chemical binding of $\mathrm{Ca}^{2+}$ to the troponin complex. Hence, in areas of high tension, deactivation of the thin filament is energetically unfavorable. We have developed the MechChem model of mechano-chemical interactions in cardiac sarcomeres around this central hypothesis. This thesis has detailed the step by step build-up and validation of the MechChem model from the capability to simulate static isometric contractions (Chapter 2) to isometric twitch contractions (Chapter 3) to isotonic twitches (Chapter 4). The MechChem model was developed for its eventual use in understanding clinically relevant problems in the human heart. To this end, MechChem was implemented within the CircAdapt model of the human heart and closed-loop circulation. As a first test, the combined model of CircAdapt with MechChem was utilized to understand the relationship between cellular- and organlevel function during heart failure and LBBB with and without CRT (Chapter 5). Overall, the latter study suggests that mechanical viability of the tissue is an important determinant of acute hemodynamic response to CRT.

\section{Comparison of previously hypothesized cooperativity mechanisms with MechChem}

The calcium concentration and resulting muscle tension data from static skinnedmuscle cell experiments are typically represented with a Hill-type fit $(1,2)$. The Hill- 
type fit uses an s-shaped curve with a steep upslope that rounds toward saturation. The experimental data presented in the study by Dobesh et al. (1) can be represented by a Hill-type curve where the steepness of the upward slope of the experimental calcium-tension relationship is well represented by this curve. However, the rounding of the top of the Hill-type curve toward saturation consistently overestimates the experimental data of Dobesh et al (1). This overestimation may be due to the fact that the Hill-type model was originally developed to understand the cooperative binding of oxygen to hemoglobin (3). Cooperative binding of oxygen to hemoglobin acts within a single molecule, and the interactions are purely chemical. This is not the case in cardiac muscle where the working range of the cooperativity mechanism, the length of the thin filament, is $1.2 \mu \mathrm{m}$ long. Aditionally, mechanical tension in the thin filament has the potential to strain the molecules. Hence, the physical explanation for the use of the Hill model is lacking in cardiac muscle. Therefore, many hypotheses have been proposed for the mechanism of cooperativity in cardiac muscle.

Some have proposed nearest neighbor cooperativity where activation of one troponin complex could promote the activation of neighboring troponin complexes, where bound $X B$ s could promote activation of neighboring troponin complexes, or where $X B \mathrm{~s}$ could promote the binding of other XBs in close proximity (4). Campbell and colleagues have proposed another near-neighbor interaction between tropomyosin molecules that mutually overlap under a troponin complex, promoting activation of neighboring troponin complexes (5). The aforementioned interactions do not account for cooperativity in a long range, so the binding of an $X B$ only promotes the binding of a neighboring $X B$. In the MechChem model, each force-generating $X B$ contributes to the tension in the thin filament from the start of the single overlap region closest to the mid line toward the z-disk. In this way, tension developed by the $X B$ in the area of the single overlap region closest to the mid line contributes to tension all along the single overlap region, thus promoting the cooperative mechanism on a long range.

In addition to nearest-neighbor cooperativity mechanisms, other groups have proposed cooperativity mechanisms that are valid over a long range. Brandt et al. 
suggested an all-or-nothing phenomenon where the mutual overlap of tropomyosin molecules under a troponin complex would result in a chain that activated or deactivated together (6). However, it is unclear how the mechanism proposed by Brandt et al. would explain the increased peak tension with incremental increases in calcium concentrations in static isometric experiments.

In addition to interactions between molecules, strain has been proposed to contribute to the cooperativity mechanism in the myocardium. Some groups have proposed that strain in the whole cell contribute to the cooperativity mechanism. They propose that the lattice of a cell has a fixed volume, so lengthening the cell would result in a thinner lattice. Hence, a myosin head would be closer to the thin filament to form an $X B(7,8)$. Others have proposed a cooperativity mechanism dependent on the strain solely within the thin filament (9). The theory proposed by Daniel et al. is that the thin filament can be stretched by up to $2 \mathrm{~nm}$ when under high stress, which could move the binding site on the thin filament within the range of a myosin head. However, both approaches apply solely to cooperative $X B$ binding and not to activation as they ignore tropomyosin.

In addition to the proposed cooperativity mechanisms regarding strain, others have attributed the mechanism of cooperativity to the number of bound XBs. With greater $X B s$, the sensitivity to calcium increases globally $(10,11)$. The global increase in calcium sensitivity leads to the ignorance of local changes in tension in the thin filament, a phenomenon that is central to the MechChem model hypothesis. A recent model by Land and Niederer (12) proposed that the troponin complex has an associated free energy related to its state dependent on the states of neighboring troponin complexes and $X B$ s. They hypothesize that the deformation of tropomyosin molecules caused by the positions of neighboring tropomyosin molecules as well as bound $X B s$ is the main determinant for whether a given troponin complex is activated. However, they do not take into account explicit effects of the tension in the thin filament. 
Contrary to all other proposed cooperativity mechanisms in cardiac tissue, we suggest a mechanism where the mechanical tension in the thin filament directly affects the chemistry. In the first developmental step of the MechChem model (Chapter 2), we simulated time-independent isometric contractions (13) to match the experimental conditions of Dobesh et al. (1). The MechChem model-simulated results fit the data of Dobesh et al.(1) better than the traditional Hill-type fit. Dobesh and colleagues noted the asymmetry in their $\left[\mathrm{Ca}^{2+}\right]$-tension data points. In an attempt to fit the data better, they utilized a modified Hill function with two cooperativity coefficients that met at the calcium concentration required for half maximal tension. The disagreement between the experimental $\left[\mathrm{Ca}^{2+}\right]$-tension data and the Hill-type fit could be because of the effects of mechanical tension in the thin filament that are not accounted for in the Hill model. Since our model with only 3 parameters fits the data better than the generally accepted method, it is possible that the mechanism we propose is the main mechanism working in the cooperativity of cardiac sarcomeres. Based on the good fit of the MechChem model results to experimental data, the novel mechanism of cooperativity we proposed could not be disproved. Hence, the motivation was provided for subsequent model development steps.

\section{Modeling sarcomere mechanics with the MechChem model}

In the physiological situation, the intracellular calcium concentration changes in time. Hence, an isometric twitch was the next logical step in model development (Chapter 3). To this end, a dynamic calcium transient, as modeled by Rice et al. (14), was introduced for time dependence. Additionally, an XB cycle model was introduced because we could no longer assume that XBs formed automatically when the thin filament was activated. We utilized a simplified two state XB cycle model to represent the detached, non-force-generating, and the attached, force-generating XB states. Simulations at various sarcomere lengths fit well with the experimental data of Janssen and Hunter (15) and Kassiri et al (16). We used these studies with their different methodologies as independent tests for the MechChem model. The ability to replicate the general qualitative results of the studies of Janssen and Hunter (15) and 
Kassiri et al. (16) provided further evidence that the mechanism of cooperativity we proposed could be the main one at work within the sarcomere. Whereas Janssen and Hunter changed sarcomere length and Kassiri et al. produced differences in peak intracellular calcium concentrations, both experimental studies showed increased duration of contraction with increased peak tension generated by the sarcomeres.

The successful test of the MechChem model in Chapter 3 led us to further develop the MechChem model to include changes in sarcomere length during contraction and relaxation in Chapter 4. To this end, we implemented the possibility of sarcomere length change within the MechChem model utilizing a Hill-type relationship (17). In the Hill-type model, the sarcomere is represented by three elements, a passive element, a contractile element, and a series elastic element. We utilized the extended model to mimic the isotonic twitch experiments of Katsnelson et al. (18) where the initial muscle length was set, and it would contract against an afterload. We tested the model against 7 afterloads, and the resulting isotonic twitch tension curves and sarcomere length curves were in good qualitative agreement with the experimental data. After this test, the logical next step for the MechChem model was to implement it within the framework of a whole heart model.

\section{MechChem modeling in the whole-heart}

By replacing the previously developed phenomenological model of cardiac sarcomere mechanics (19) in CircAdapt with the more physics and physiology-based MechChem model, the model could be utilized to further understand the relationship between cellular-level function and whole organ function. Upon implementation of the MechChem model within the CircAdapt modeling framework, we have conducted a first test (Chapter 5) to understand the relationship between cell-level and organ-level function in heart failure and LBBB with and without CRT. The results showed that electro-mechanical dyssynchrony, represented by septal rebound stretch, was reduced when cross-bridge cycling rates were lower. This finding could help us interpret tissue strain patterns in heart failure patients with LBBB because the reduced septal rebound stretch would mean reduced mechanical viability in the tissue related to lower cross- 
bridge cycling rates. The study presented in Chapter 5 was a first test for the combined model. This work paved the way for more multi-scale modeling studies in the future, some of which are outlined in the Future Perspectives section.

\section{Critical appraisal of the MechChem model}

In this thesis, we developed a computational model of cardiac sarcomere contraction that centered around a single mechanism of cooperativity. It is likely that we left out some mechanisms of cooperativity that may be important in cardiac contraction. However, due to the ability of the MechChem model to reproduce results from many different experimental setups, it is possible that the mechanism presented in this thesis is the main mechanism working in cardiac muscle.

Further, each development step of the MechChem model has been assessed using data recorded in different experimental conditions. All of the experimental data we compared to in Chapters $2-4$ came from rats. The time-independent skinned muscle cell experiments by Dobesh et al. (1) were performed on RV cardiac trabeculae at a temperature of $15{ }^{\circ} \mathrm{C}$ (Chapter 2). The experimental data used for comparison in Chapter 3 differed from the study of Dobesh et al. as the muscles were intact instead of skinned, and the temperatures of the experiments of Jannsen and Hunter (15) and Kassiri et al (16) were $23-24^{\circ} \mathrm{C}$ and $20-22^{\circ} \mathrm{C}$, respectively. In Chapter 4 , the experimental results of Katsnelson et al. were used for comparison (18). The data were collected from LV papillary muscles at $29^{\circ} \mathrm{C}$. It has been shown that changes in temperature have a large effect on results in experiments on sarcomeres (20), which may explain why the MechChem model needed re-parameterization for comparison to each experimental setup. Although re-parameterization was necessary due to different experimental conditions for the data we compared to, the underlying physical principles governing the equations of the model did not change.

While the underlying physical principles determining the MechChem model behavior did not change between development steps, new components were introduced to the MechChem model with each development step. While the MechChem parameters 
changed throughout each new development step of the model, the underlying physical principles remained consistent. The underlying physical principles are conserved across species, so the MechChem model that was previously parameterized for rat could be parameterized for human tissue. Hence, implementation of the MechChem model in the CircAdapt modeling framework of the human closed-loop circulation can be viewed as a test for the model. In Chapter 5, the myocardial tissue strain patterns calculated by the MechChem model within CircAdapt are comparable to human LBBB patients. Hence, we can conclude that the general principles and hypothesis of the MechChem model cannot be rejected based on the work presented in this thesis.

Additionally, the studies presented in Chapters 3, 4, and 5 all utilize a prescribed calcium transient to represent the dynamic free intracellular calcium concentration. Hence, the calcium transient is the same for every beat without the feedback of force on the calcium transient. It has also been shown that atrial cells have different calcium transients than ventricular cells, mainly due to the lack of t-tubules in atrial cells (21). It will be important in future research to utilize calcium transients that are more specific to the different cell types. Additionally, the TriSeg model in CircAdapt used in this thesis (Chapter 5) utilizes a simplified geometry (19). Each wall is represented as a single muscle fiber (22), so tissue properties are not varied throughout a wall. Despite this limitation, the simplified geometry still produced results that were comparable to clinical measurements of regional myocardial deformation in patients with dyssynchronous heart failure.

\section{Model-generated testable hypotheses}

The MechChem model results have provided potentially testable hypotheses on the biophysical working mechanism of sarcomere contraction and relaxation. In the timeindependent skinned isometric contraction results (Chapter 2), it was shown that it was more likely that a troponin would be activated in areas of the single overlap region closer to the z-disk because those were under the greatest degree of tension. The aforementioned hypothesis may be testable with an experimental setup similar to 
that by Sun et al. (23) where regions of troponin C were labeled with fluorescent probes. When the troponin complex was activated, the probes would change orientation. However, Sun et al. admitted that the probes they place on the troponin complex could alter the effect of tension on calcium binding. If alternative probes could be found that would not interfere with calcium binding based on tension, the directionality of activation could be viewed under areas of high tension.

In addition to the predicted heterogeneity in troponin complex activation along the thin filament, the MechChem model predicted heterogeneity in $X B$ binding along the thin filament. The results of Chapter 3 suggest that $X B$ s are more likely to bind in areas of the thin filament under high tension. Additionally, when the calcium concentration decreases in the intracellular space, the MechChem model predicts that relaxation begins in the single overlap region closer to the mid line where the thin filament is under low mechanical tension. Desai et al. have conducted experiments observing the binding of a myosin head to the thin filament (24). Their results showed no directionality in $X B$ binding. However, the myosin heads were not tethered to a thick filament, so they could not generate force. If their experimental setup could be altered to utilize tethered myosin heads that could generate force, the setup could provide a way to test our hypothesis.

\section{Future perspectives and applications}

This thesis has detailed the build-up of the model of mechanics of the cardiac sarcomere. In the future, it will be important to develop a better understanding of the electrics. It has been widely known since the seminal work of Ringer (25) that calcium induces muscle contraction. Throughout the years, research was done to better understand the dynamics of calcium handling in the myocardium and the relationship between calcium and muscle contraction, known as excitation-contraction coupling. In the studies in this thesis, a representative calcium transient was utilized beginning in Chapter 3 when the MechChem model was extended to mimic an isometric twitch. The dynamic calcium transient prescribed in the MechChem model can be replaced with a model of calcium handling. This is especially important in view of the feedback 
of force on the intracellular calcium transient that is apparent in experiments on myocardium (26-29) but currently not included in the MechChem model representation of the calcium transient.

With the implementation of a calcium handling model, we can even look another level deeper. The calcium concentration in the cytoplasm is determined by the flow of ions through multiple channels and pumps. There are many possibilities for genetic mutations in any one of those channels or pumps in the heart, possibly resulting in changes to the activation of tissue in the whole heart. The implementation of MechChem in CircAdapt enables implementation of a calcium handling system model as well. When that is complete, we can begin understanding tissue- and organ-level consequences of abnormalities at the genetic level.

In Chapter 5, we introduced a proof of concept study in which the MechChem model was implemented within the CircAdapt framework. The implementation of a model of calcium handling within this framework will be necessary for future studies. For example, the morphology of the calcium transient has been shown to differ between healthy and failing hearts (30). The current calcium handling model does not output a different calcium transient based on the underlying conditions.

In the whole heart, the calcium transient is initiated by an action potential. Re-entrant waves that depolarize the myocardium can cause problems such as atrial fibrillation. The current CircAdapt model determines activation times for each piece of tissue, triggering the calcium transient for that section of tissue. In the future, CircAdapt can be coupled to an electrophysiology model such as the model developed by Grandi et al. (31) to model the human ventricular action potential. In a model of the action potential, individual ion currents determine the voltage of the cell. The currents activated throughout the action potential eventually trigger calcium release from the sarcoplasmic reticulum. The addition of an action potential model may be useful in understanding the connection between depolarization in the tissue and the resulting calcium transient. 
Multi-scale modeling provides the possibility to understand interactions between spatial scales. So far, the future perspectives have focused on changes in calcium transient morphology and the action potential and how the changes scale up to wholeheart mechanics and hemodynamics. Utilizing the model, it is also possible to focus on whole-heart mechanics and scale down to tissue-level mechanics. The Frank-Starling law of the heart results in a more forceful contraction when preload is higher. This is especially important during ventricular conduction abnormalities such as LBBB, where late-activated tissue is pre-stretched by early-activated tissue. We have shown in Chapter 3 that the duration of contraction is highly dependent on the peak tension developed in the sarcomere. Hence, pre-stretched tissue will contract longer and more forcefully. This can contribute to greater levels of dyssynchrony, but only when the tissue remains mechanically viable (Chapter 5). It may also be interesting to look at our new mechanism in hypertensive conditions where left or right ventricular afterloads are chronically heightened. It has been shown by Chirinos and colleagues $(32,33)$ that high afterloads in late systole can cause diastolic dysfunction in humans. A higher afterload late in systole would require the tissue to generate more tension to overcome the high afterload. High peak tension in the thin filament leads to a prolongation of contraction in the muscle (Chapter 3). Hence, the systolic phase may last longer due to prolonged contraction of the sarcomeres. In turn, there is less time for the diastolic phase of the cardiac cycle. Prolongation of contraction could result in altered filling patterns in the ventricles, eventually leading to diastolic heart failure. This could be an interesting test for the model in the future.

\section{General Conclusions}

In this thesis, we aimed to understand the mechanics of cardiac sarcomere cooperativity and contraction. We developed a sarcomere contraction model based on the novel hypothesis of the mechanism of cooperativity in cardiac sarcomeres. We proposed that there is an intrinsic chemical cooperativity in the thin filament that is boosted by mechanical tension locally along the thin filament. As a result of the proposed mechanism, high tension in the thin filament both promotes activation of 
the troponin complexes early in contraction and hinders relaxation in the thin filament. The MechChem model has successfully reproduced time-independent isometric contraction experiments, isometric twitch experiments, and isotonic twitch experiments. The model has led to two main predictions. 1) Cross-bridges form heterogeneously along the thin filament with increasing probability of a strongly bound cross-bridge in areas of high tension closer to the z-disk. 2) High tension locally on the thin filament not only increases the probability of strongly bound cross-bridges but also increases the strength of the bond of calcium to troponin. Higher developed tension in the thin filament is responsible for the prolongation of contraction seen in isometric twitch experiments.

While the MechChem model was developed and validated with experimental data from isolated cells, the eventual goal was the use of the model to help understand the mechanical function of the whole heart. Hence, MechChem has been implemented within the framework of the CircAdapt model of the closed-loop circulation to simulate the failing human heart with asynchronous cardiac activation due to left bundle branch block in the human heart. Simulations have shown that septal rebound stretch is a strong indicator for cardiac resynchronization therapy response. Further, we have shown that decreased septal rebound stretch could be due to the decrease in mechanical viability of the tissue, represented in our simulations as a reduction in the cross-bridge cycling rate. The reduction in cross-bridge cycling rate provides an explanation for why cardiac resynchronization therapy would not be successful in heart failure patients with left bundle branch block but little septal rebound stretch. An electrical treatment such as cardiac resynchronization therapy will not solve this mechanical dysfunction. Given these results, the addition of the MechChem model to the CircAdapt framework is a promising step forward in multi-scale and multi-physics modeling of cardiovascular dynamics. The model will be a powerful tool in the future to develop hypotheses with regards to mechanical dysfunction at the cellular level in cardiac dysfunction. 


\section{References}

1. Dobesh DP, Konhilas JP, de Tombe PP. Cooperative activation in cardiac muscle: impact of sarcomere length. American Journal of Physiology - Heart and Circulatory Physiology. 2002;282(3):H1055H62.

2. Kentish JC, ter Keurs HE, Ricciardi L, Bucx JJ, Noble MI. Comparison between the sarcomere length-force relations of intact and skinned trabeculae from rat right ventricle. Influence of calcium concentrations on these relations. Circulation Research. 1986;58(6):755-68.

3. Hill AV. The Combinations of Haemoglobin with Oxygen and with Carbon Monoxide. I. Biochemical Journal. 1913;7(5):471-80.

4. Razumova MV, Bukatina AE, Campbell KB. Different Myofilament Nearest-Neighbor Interactions Have Distinctive Effects on Contractile Behavior. Biophysical Journal. 2000;78(6):3120-37.

5. Campbell SG, Lionetti FV, Campbell KS, McCulloch AD. Coupling of Adjacent Tropomyosins Enhances Cross-Bridge-Mediated Cooperative Activation in a Markov Model of the Cardiac Thin Filament. Biophysical Journal. 2010;98(10):2254-64.

6. Brandt PW, Diamond MS, Rutchik JS, Schachat FH. Co-operative interactions between troponintropomyosin units extend the length of the thin filament in skeletal muscle. Journal of Molecular Biology. 1987;195(4):885-96.

7. Tanner BCW, Farman GP, Irving TC, Maughan D W, Palmer BM, Miller M S. Thick-to-Thin Filament Surface Distance Modulates Cross-Bridge Kinetics in Drosophila Flight Muscle. Biophysical Journal. 2012;103(6):1275-84.

8. Williams CD, Salcedo MK, Irving TC, Regnier M, Daniel TL. The length-tension curve in muscle depends on lattice spacing. Proceedings of the Royal Society B: Biological Sciences. 2013;280(1766):20130697.

9. Daniel TL, Trimble AC, Bryant Chase P. Compliant Realignment of Binding Sites in Muscle: Transient Behavior and Mechanical Tuning. Biophysical Journal. 1998;74(4):1611-21.

10. Izakov V, Katsnelson LB, Blyakhman FA, Markhasin VS, Shklyar TF. Cooperative effects due to calcium binding by troponin and their consequences for contraction and relaxation of cardiac muscle under various conditions of mechanical loading. Circulation Research. 1991;69(5):1171-84.

11. Landesberg A, Sideman S. Mechanical regulation of cardiac muscle by coupling calcium kinetics with cross-bridge cycling: a dynamic model. American Journal of Physiology. 1994;267(2):H779-H95.

12. Land S, Niederer SA. A Spatially Detailed Model of Isometric Contraction Based on Competitive Binding of Troponin I Explains Cooperative Interactions between Tropomyosin and Crossbridges. PLoS Computational Biology. 2015;11(8):e1004376.

13. Dupuis LJ, Lumens J, Arts T, Delhaas T. Mechano-chemical Interactions in Cardiac Sarcomere Contraction: A Computational Modeling Study. PLOS Computational Biology. 2016;12(10):e1005126.

14. Rice JJ, Wang F, Bers DM, de Tombe PP. Approximate Model of Cooperative Activation and Crossbridge Cycling in Cardiac Muscle Using Ordinary Differential Equations. Biophysical Journal. 2008;95(5):2368-90.

15. Janssen PM, Hunter WC. Force, not sarcomere length, correlates with prolongation of isosarcometric contraction. American Journal of Physiology. 1995;269(2):H676-H85.

16. Kassiri Z, Myers R, Kaprielian R, Banijamali HS, Backx PH. Rate-dependent changes of twitch force duration in rat cardiac trabeculae: a property of the contractile system. The Journal of Physiology. 2000;524(Pt 1):221-31.

17. Hill AV. The Heat of Shortening and the Dynamic Constants of Muscle. 1938;126(843):136-95.

18. Katsnelson LB, Nikitina LV, Chemla D, Solovyova O, Coirault C, Lecarpentier Y, et al. Influence of viscosity on myocardium mechanical activity: a mathematical model. Journal of Theoretical Biology. 2004;230(3):385-405.

19. Lumens J, Delhaas T, Kirn B, Arts T. Three-Wall Segment (TriSeg) Model Describing Mechanics and Hemodynamics of Ventricular Interaction. Annals of Biomedical Engineering. 2009;37(11):2234-55.

20. de Tombe PP, Stienen GJM. Impact of temperature on cross-bridge cycling kinetics in rat myocardium. The Journal of Physiology. 2007;584(Pt 2):591-600.

21. Smyrnias I, Mair W, Harzheim D, Walker SA, Roderick HL, Bootman MD. Comparison of the Ttubule system in adult rat ventricular and atrial myocytes, and its role in excitation-contraction coupling and inotropic stimulation. Cell Calcium. 2010;47(3):210-23. 
22. Arts T, Bovendeerd PH, Prinzen FW, Reneman RS. Relation between left ventricular cavity pressure and volume and systolic fiber stress and strain in the wall. Biophysical Journal. 1991;59(1):93-102.

23. Sun Y-B, Lou F, Irving M. Calcium- and myosin-dependent changes in troponin structure during activation of heart muscle. The Journal of Physiology. 2009;587(Pt 1):155-63.

24. Desai RA, Geeves MA, Kad NM. Using Fluorescent Myosin to Directly Visualize Cooperative Activation of Thin Filaments. Journal of Biological Chemistry. 2014.

25. Ringer S. A further Contribution regarding the influence of the different Constituents of the Blood on the Contraction of the Heart. The Journal of Physiology. 1883;4(1):29-42.

26. Backx PH, Ter Keurs HE. Fluorescent properties of rat cardiac trabeculae microinjected with fura2 salt. American Journal of Physiology - Heart and Circulatory Physiology. 1993;264(4):H1098-H110.

27. ter Keurs HEDJ, Wakayama Y, Miura M, Shinozaki T, Stuyvers BD, Boyden PA, et al. Arrhythmogenic $\mathrm{Ca} 2+$ release from cardiac myofilaments. Progress in Biophysics and Molecular Biology. 2006;90(1-3):151-71.

28. Jiang $\mathrm{Y}$, Patterson MF, Morgan DL, Julian FJ. Basis for late rise in fura $2 \mathrm{R}$ signal reporting [Ca2+]i during relaxation in intact rat ventricular trabeculae. American Journal of Physiology - Cell Physiology. 1998;274(5):C1273-C82.

29. Kurihara S, Komukai K. Tension-dependent changes of the intracellular Ca2+ transients in ferret ventricular muscles. The Journal of Physiology. 1995;489(Pt 3):617-25.

30. Kirk JA, Kass DA. Cellular and Molecular Aspects of Dyssynchrony and Resynchronization. Cardiac electrophysiology clinics. 2015;7(4):585-97.

31. Grandi E, Pasqualini FS, Bers DM. A novel computational model of the human ventricular action potential and Ca transient. Journal of Molecular and Cellular Cardiology. 2010;48(1):112-21.

32. Chirinos JA, Phan TS, Syed AA, Hashmath Z, Oldland HG, Koppula MR, et al. Late Systolic Myocardial Loading is Associated With Left Atrial Dysfunction in Hypertension CLINICAL PERSPECTIVE</span>. Circulation: Cardiovascular Imaging. 2017;10(6):e006023.

33. Chirinos JA, Segers P, Duprez DA, Brumback L, Bluemke DA, Zamani P, et al. Late Systolic Central Hypertension as a Predictor of Incident Heart Failure: The Multi-Ethnic Study of Atherosclerosis. Journal of the American Heart Association. 2015;4(3). 



\section{Summary}

The heart is a pump that propels blood throughout the body. This pump function is enabled by the rhythmic contraction and relaxation of the cardiac muscle developing tension in the cardiac walls. The most basic functional unit of muscle, called the sarcomere, is responsible for the active generation of force through biophysical interactions between thick and thin filaments. Myosin heads that protrude from the thick filaments can attach to myosin binding sites on thin filaments, forming crossbridges (XB) that can generate force. At rest, binding sites on the thin filaments are blocked. Electrical signals initiate depolarization of myocardial cells, triggering the release of calcium into the intracellular space. Muscle is activated when a calcium ion $\left(\mathrm{Ca}^{2+}\right)$ binds to a molecule called troponin, triggering conformational changes of the thin filament that result in the unblocking of the binding sites.

It has been shown that slight increases in the intracellular calcium concentration result in disproportionately large increases in tension. While it is widely accepted that the disproportionate increase in tension is due to cooperativity, the mechanism is debated. In this thesis, we propose a novel, physics- and chemistry-based mechanism of cooperativity in the cardiac sarcomere and develop a computational model to test it. Because the model is made to understand clinically relevant questions, it is built up and validated step by step for its eventual implementation within a model of the closed-loop circulation.

Groups have performed experiments in which isolated cardiac muscle cells were skinned and placed in a bath containing specific concentrations of ions. The muscle cells developed tension while their length was held constant, and the developed steady-state tension was recorded. The experiment was conducted multiple times with different calcium concentrations resulting in a group of data points that characterized the relationship between calcium concentration and tension for a given 
sarcomere length. The calcium-tension relationship from these experiments is typically fitted with a sigmoidal curve. The curve generally has a steep upslope in tension with small changes in calcium concentration, implying cooperativity. In Chapter 2, we present our novel hypothesis for the mechanism of cooperativity in the cardiac sarcomere. We hypothesize that high mechanical tension in the thin filament hinders the release of calcium from the troponin complex, thus hindering deactivation of the muscle. We propose that a baseline level of chemical cooperativity intrinsic to the thin filament is boosted by mechanical tension. Based on our hypothesis, we have developed a computational model of mechano-chemical interactions in the cardiac sarcomere, called MechChem. We utilized the MechChem model to mimic the conditions in the steady-state isometric contraction experiments. The MechChem model produced calcium-tension curves that fit experimental data better than the typically used sigmoidal curve, so our hypothesis could not be disproved. In addition, simulation results provided a potentially testable hypothesis because our model predicted the existence of a spatial heterogeneity in $\mathrm{XB}$ binding, with more $\mathrm{XBS}$ forming in areas of the thin filament under greater tension.

The ability of the MechChem model to successfully reproduce data from skinned muscle cell experiments provided the motivation to develop the model further. In a physiological situation, the calcium concentration in the intracellular space does not remain constant but rises and falls in time. The increase in calcium concentration triggers contraction, and the subsequent decrease results in relaxation. In Chapter 3, we extended the MechChem model to include transient changes in calcium concentration. Additionally, we introduced a model of the cross-bridge cycle in which myosin heads can cycle between an attached, force-generating state and a detached, non-force-generating state. We utilized the model to mimic isometric twitch experiments in which the sarcomere length was held constant while the calcium concentration changed in time. Experiments have shown that the duration of the relaxation phase of contraction increases with greater developed peak tension during a twitch. MechChem simulation results suggest that the prolongation of the relaxation 
phase at higher tension is a result of the tighter binding of a calcium ion to troponin in areas under higher tension, thus delaying the deactivation of troponin.

In Chapter 4, we extended the MechChem model further to include the ability of the sarcomere to shorten. Model simulations were performed where the initial sarcomere length was set, and the sarcomere contracted against various afterloads. When the sarcomere generated enough tension to overcome the afterload, it would shorten. When it no longer developed enough tension, the afterload would cause relengthening of the sarcomere, mimicking isotonic twitch experiments. Modelgenerated results were strikingly similar to experimental results. This was the final developmental step before the model was ready to be integrated within a whole-heart model.

In Chapter 5, the MechChem model was integrated within the CircAdapt model of the heart with its closed-loop circulation. The combined model was utilized in an attempt to understand the connection between cross-bridge kinetics and whole-heart function of heart failure patients with left bundle branch block (LBBB) before and after cardiac resynchronization. Our results suggested that while regional ventricular tissue deformation patterns appeared more synchronous and uniform in patients with a reduced cross-bridge cycling rate, their potential for improved outcome after cardiac resynchronization is significantly compromised. In addition, the simulated patterns of left ventricular mechanical discoordination in the electrically dyssynchronous heart showed the same typical characteristics as observed clinically in patients. These results suggest that the integration of the MechChem model within the CircAdapt framework is a promising advancement in multi-scale modeling of cardiovascular dynamics. Moving forward, the integrated model will be a powerful hypothesis-generating tool when understanding cellular level mechanical dysfunction in relation to whole-heart hemodynamics. 



\section{Valorisation}

Cardiovascular dysfunction is a leading cause of death in the western world according to the World Health Organization. Despite centuries of research, there still remain gaps in the current understanding of the cardiovascular system. In order to understand the complexities of cardiac function in both healthy and pathological situations, computational models have been developed to mimic various biophysical aspects of the circulation.

Of the various aspects of the circulation, this thesis centers on the development of a novel computational model of the most basic functional unit of the contractile system, called the sarcomere. The computational model presented in this thesis, the MechChem model, was built for its eventual use in understanding clinically relevant problems in the human heart. The emphasis on clinical translation means that this project was specifically designed with valorisation in mind. This chapter will focus on the valorisation opportunities originating from the research presented in this thesis.

\section{Knowledge transfer}

Knowledge gained through research, whether the data is positive or negative, is valuable to the scientific community. Distribution of knowledge can save both time and money. If knowledge and data are freely available, the number of experiments repeated by different research groups could potentially be decreased. Additionally, the distribution of knowledge allows others to build on existing ideas and hypotheses. Hence, knowledge transfer for further utilization is a key aspect of valorisation. To this end, the work described in this thesis has been presented at international conferences and workshops. Furthermore, each of the chapters (Chapter 2-5) detailing the buildup of the MechChem model as well as its application have been accepted for publication 
in scientific journals with the purpose of making the studies available to the scientific community.

In addition to the publication of the central hypotheses and results in this work, the source code of the computational models described in this thesis has been made openly available online. Chapter 2 details our novel hypothesis of cooperative activation in the cardiac sarcomere. The availability of the source code makes it possible for other groups to utilize the model that represents the main hypothesis underlying MechChem with their own contraction models. Chapter 3 builds on the MechChem model by adding a simplified contraction model for which the source code is also freely available online. This work can also be utilized by other groups wishing to insert a simplified contraction model in a larger model of the heart. Overall, the availability of the source code provides possibilities for others to build on the work presented in this thesis, thus increasing the possible knowledge gained from this work.

Prior to the development of the MechChem model, a relatively simple model of the closed-loop circulation, called CircAdapt, has been developed at Maastricht University. The model is composed of various modules that represent the components of the circulation. A user-friendly interface, developed previously for use of the CircAdapt model as an educational tool, is freely available on www.circadapt.org. This educational tool enables real-time beat-to-beat simulation of various hemodynamic signals, and its interface allows easy modification of model parameters. As a result, one can learn about cardiac physiology by tweaking different properties of the circulation. The educational tool is part of the medical curriculum in Maastricht University. In the future, the MechChem model may be included in the educational tool. This would allow students to better understand properties of the heart at the tissue level and how they determine regional myocardial mechanics, which can be measured clinically using echocardiographic deformation (or strain) imaging. Regional myocardial strain patterns provide information on regional tissue properties such as myocardial stiffness and contractility that can be utilized to pinpoint the underlying causes of cardiac dysfunction. Further, this information may provide insight to which 
treatment option to choose. Hence, the addition of the MechChem model to the CircAdapt educational tool, and further the medical curriculum, may help future clinicians gain deeper understanding of the cardiac abnormalities they will eventually treat. Because the additional knowledge gained through the use of the MechChem model benefits clinicians, it will also benefit the patients who will be receiving treatments.

\section{Benefits of a model-based approach}

When integrated in a multi-scale model of the human heart and circulation, such as CircAdapt, the well-validated MechChem model provides the ideal environment for in silico cardiovascular research. As such, it can be utilized as a hypothesis-generating tool. The in silico development of a more targeted hypothesis and study design prior to animal experimentation can reduce the number of animals necessary for research. In addition to the potential reduction in animals used for experiments due to computational modeling, the cost will also be reduced, allowing allocation of financial resources to further research projects.

While computational modeling can potentially decrease the number of experiments necessary on animals, it also provides a platform to isolate and change specific parameters in a simple and fast way that may not be possible in an animal model. The study presented in Chapter 5 of this thesis is an example of the latter. We changed a single parameter to allow us to understand the effect of a reduced rate of cross-bridge cycling, independent of any other interactions, on mechanical dyssynchrony in patients with dyssynchronous heart failure. To perform the same reduction in rate of contraction in animals, a certain drug may need to be administered or animals would need to be bred to have specific traits. Utilizing a computational model also allows the changes to be made in a short period of time, thereby saving time to generate results. Reduction in the intervention on animals, in addition to saving time and money, may also reduce ethical concerns. A computational model can be pushed to absolute extremes without any inhumane actions. While computational modeling cannot 
replace experiments on animals in the foreseeable future, it can reduce the amount of experiments that need to be performed by helping to create a more targeted hypothesis for experimentation.

\section{Clinical applications}

In this thesis (Chapter 5), we have utilized the MechChem model embedded in the CircAdapt model of the closed-loop circulation to understand a clinical problem. Previous studies have shown that the deformation pattern of tissue in the septal wall of the heart can serve as an indication for whether a heart failure patient will respond favorably to cardiac resynchronization therapy. In our study in Chapter 5, we tried to understand the underlying mechanisms responsible for the different septal deformation patterns in a specific cardiac abnormality known as left bundle branch block. The results in Chapter 5 are hypothesis-generating and also have potential clinical applicability. It may be possible to utilize the model in the future to understand septal strain patterns in the individual heart failure patient with the possibility to aid in the determination and personalization of therapy. The study presented in Chapter 5 was a first test of the multi-scale CircAdapt-MechChem model, but the potential impact will be broader. As the model is validated and improved, it may be utilized to study a wider range of cardiac abnormalities. The model could be made available to clinicians to help them make decisions regarding patient prognosis and treatment options. The model can be fed personalized information such as ECG-based activation times as well as tissue deformation patterns in different segments of the heart. Hence, the model has the potential to affect treatment in the clinic by aiding the clinician with decision-making, eventually improving the standard of care that patients receive. 




\section{Acknowledgements}

Completing a $\mathrm{PhD}$ here in Maastricht has proven a rewarding experience filled with ups and downs, and it would not have been possible without those of you who have been there for me along the way. Throughout my years as a PhD student, many of you have taught, helped, supported, and encouraged me. I am grateful to every one of you.

First and foremost, I would like to thank my supervisors for everything. Tammo, you have put so much time into this project including all of the meetings, scientific discussions, and paper editing. You always provide a critical opinion and push for improvement. I have learned so much from you including how to snowboard! I would also like to recognize my co-promoter (and voice of reason), Joost, for all of the time you have put into this project. Thanks for everything both in and outside of the office. It has been a lot of fun to enjoy not only scientific discussions, but wine, jokes, and barbeques as well. Theo, you have contributed so much to this project including countless ideas. You always thought everything through several steps in advance, so you were always ready for any question I could throw at you. You have invested your time and effort to help me and to make sure that I could progress. This project would never have been possible without you. I have really enjoyed all of our discussions (including the non-scientific ones).

Bill Hunter, you have inspired, encouraged, taught, and guided me. I am truly grateful for your continued mentorship and interest in my career. Frits, your valuable input and discussions about LBBB are greatly appreciated. Gudrun and Patrick, you have been our go-to calcium handling experts. To all of the other I'MCARIM PhD representatives, it has been great to serve alongside you. 
I would like to extend a special thanks to all of my colleagues in BME who have made a great environment in the department throughout my years here. Claire, you keep the whole department together and make sure we never do anything too stupid. Jeroen, you are always willing to provide technical help. Koen (Kuku), I have really enjoyed all of our chats about life. I would like to extend a special thanks to the Lumens crew (Joost, John, Aurore, Peter, Erik, Tijmen, and Nick) for all of the discussions, beers, and BBQs. Ben and Frank, you are the fruit club's most awesome members and best Friday song enthusiasts. I want to send a special shout out to Bennis and Niek for the jam sessions! I also want to thank Sjeng and Maarten for all of our beautiful \#coffeemoments. I have had so many fun chats with Pamir, Arnold, Wouter, and Raoul. I wish the best of luck to the newest PhD students in our department, Myrthe and Mehrdad. Bibi, Rosa, Yeshi, and Job, you guys were super fun students. Georgina (Gi), Jeire (Jeire-bomb), and Raf, all of the dinners and trips to Valkenburg have been great. Bart, thanks for all of the beers, BBQs, and Mestreeeeechse noises. Elien, I could always count on you for nice chats, tennis watching, and hilarious comments. Finally, I need to extend a very special thank you to my office mates Aurore and Marije for creating a nice atmosphere, dealing with my shenanigans, and all of the fun times outside of work.

I would like to extend a special thanks to my BME bestie and paranymph, Peter (Pierre). You really made my time in the department as fun and lovely as it could possibly be. We, the dream team, got up to so much stupid stuff, took trips all over the world, had countless BBQs, dinners, beers, wine, hilarious moments, pranks, and even occasional serious chats. Sabine, all of the dinners and wine have been so much fun, and I need to extend a special thanks [and sorry] for putting up with our ridiculous antics.

Another special thanks goes out to my paranymph, Margaux (Gaux). We have been doing crazy stuff together since the moment we met, and it's made my time in Maastricht amazing. Thanks for all of the awesome trips we took, for coming to my hometown, for constantly making me laugh (and spit beer), SHAKESPEARE, Dobby, 
Mona (your absolute fav), "Rarmony", the parties, shots, dinners, shenanigans, and for being there in the serious moments. To the member completing Team Triangle, Kim (Kiks), we could always count on you for great times, face zoom-ins, coffee breaks, discussions, wine nights, movie nights, a voice of reason, your love for peanuts, and Limburg lessons. The nights out and the movies with the rest of the pathology crew (special shout out to Olivia, Jan, and Jenny) have been really fun!

Judith (J.C.) and Paula, thanks for being super awesome scientists with a tequila twist!

I absolutely must send a giant grazie to Little Italy down the hall. First, obviously, I must recognize Andrea (L'uomo) for being the best. To the others in the cardio crew (Raquel, Vasco, Christina, Nico, Zenab, Martina, and Indira), you guys are a great lunch and party crew. Steffi and Leni, thanks for all of the coffee breaks and PhD club nights! Bea and Fede, we have shared so many fun times, cooking evenings, parties, and trips to Italy! Marida, you are my true espresso buddy and great person to talk to. Dr. Siracusano, you are the most amazing dog-box buddy ever! Lara, I am deeply thankful for the friendship we share. Thank you for being my wine buddy and my sounding board. I have super enjoyed all of the dinners, drinks, salty couscous, spritz optimization, \#barolomoments, and especially all of our chats.

Thanks to my original Maastricht crew! Daniel, you are the true BBQ master! Michael, you're a totally Skye beer buddy. Jennifer (Jenny Stardust), you are generally awesome, you fix all of the Picasso's, and you're never 'geein me the boke'. John (Johnny boy), you have been there since day 1 , provided great advice, served as a brilliant beer buddy, and made the best burritos in Europe. Hazel, I have really enjoyed all of the dinners and wine! Rens, you are a great whiskey buddy. Elke, my dear friend, it has been a wild ride. Thank you for always having my back, sharing dinosaurs, all the beers, the sober fun, and of course the epic Marschings.

Tina, Georg, and Natalie, I have enjoyed all of the walks, nerd movie days, dinners, and holidays. Andrea and Paolo, thanks for all of the documentary nights, wine, dinners, and North American evenings! Monika and Nicola, you have gone through 
this whole process with me (even if you didn't want to at first), and thanks for all of the trips, wine, Italian lessons, cooking lessons, and discussions.

Thanks to all of my New Jersey people for being there for me no matter where I go! Mike and Ashley, you guys provide endless laughter. Tarin and Matt (M-Dawg), you guys are always there for me when I need someone to talk to. Tammy (Tamster), thanks for putting up with me. Katherine and Marty, you have always given me advice and support. Cheers to my Doyles's/Karnaval crew (Nick, Michelle, Angelina, Pat, and Brittany) for the ridiculous times. I would like to extend a shout out to my NJIT buddies Megan, Breanna, and Breanne for all of the Skype sessions, advice, and jokes!

Finally, I would like to thank my wonderful family for always being there for me. Mom and Dad, you always support and encourage me (and run me back and forth to the airport). There is absolutely no way I could have done this without you guys. Michelle and Dan, you support me and send me all the photos of my totally adorable niece and nephew. Annabelle and Daniel, it's amazing to see the two of you progress, and I can't wait to see what you will accomplish as you grow up.

Thank you all. 




\section{Curriculum vitae and publications}

\section{About the author}

Lauren J. Dupuis was born on July 26, 1990 in Toms River, New Jersey. She completed high school in June 2008 and began work toward her Bachelor degree at New Jersey Institute of Technology in September 2008. Lauren graduated summa cum laude with her B.Sc. degree in Biomedical Engineering in May 2012. Thereafter she moved to Auckland and started in January 2013 her master's research project entitled The Mechano-Energetics of the Heart under the supervision of Professor Martyn Nash, Dr. Denis Loiselle, Dr. David Nickerson, and Dr. Kenneth Tran. With the successful completion of this project, Lauren completed her Master of Engineering at the University of Auckland with First Class Honours in January 2014 and was awarded her degree thereafter. In March 2014, Lauren began her PhD research in the department of Biomedical Engineering at Maastricht University (CARIM School for Cardiovascular Diseases, Maastricht). There she helped develop the MechChem model of mechanochemical interactions in the cardiac sarcomere under the supervision of Professor Tammo Delhaas, Professor Theo Arts, and Dr. Joost Lumens.

\section{List of Publications}

Dupuis L, Lumens J, Arts T, Delhaas T. Mechano-chemical Interactions in Cardiac Sarcomere Contraction: A Computational Modeling Study. PLOS Computational Biology. 2016;12(10):e1005126.

Dupuis LJ, Lumens J, Arts T, Delhaas T. High Tension in Sarcomeres Hinders Myocardial Relaxation. PLOS One. (Accepted).

Dupuis L, Lumens J, Arts T, Delhaas T. Mechano-chemical Interactions in Cardiac Sarcomere Shortening. Computing in Cardiology. 2018.

Dupuis L, Lumens J, Prinzen F, Arts T, Delhaas T. Linking Cross-bridge Cycling Kinetics to Cardiac Resynchronization Therapy: A Multiscale Modelling Study. EP Europace. (Accepted). 


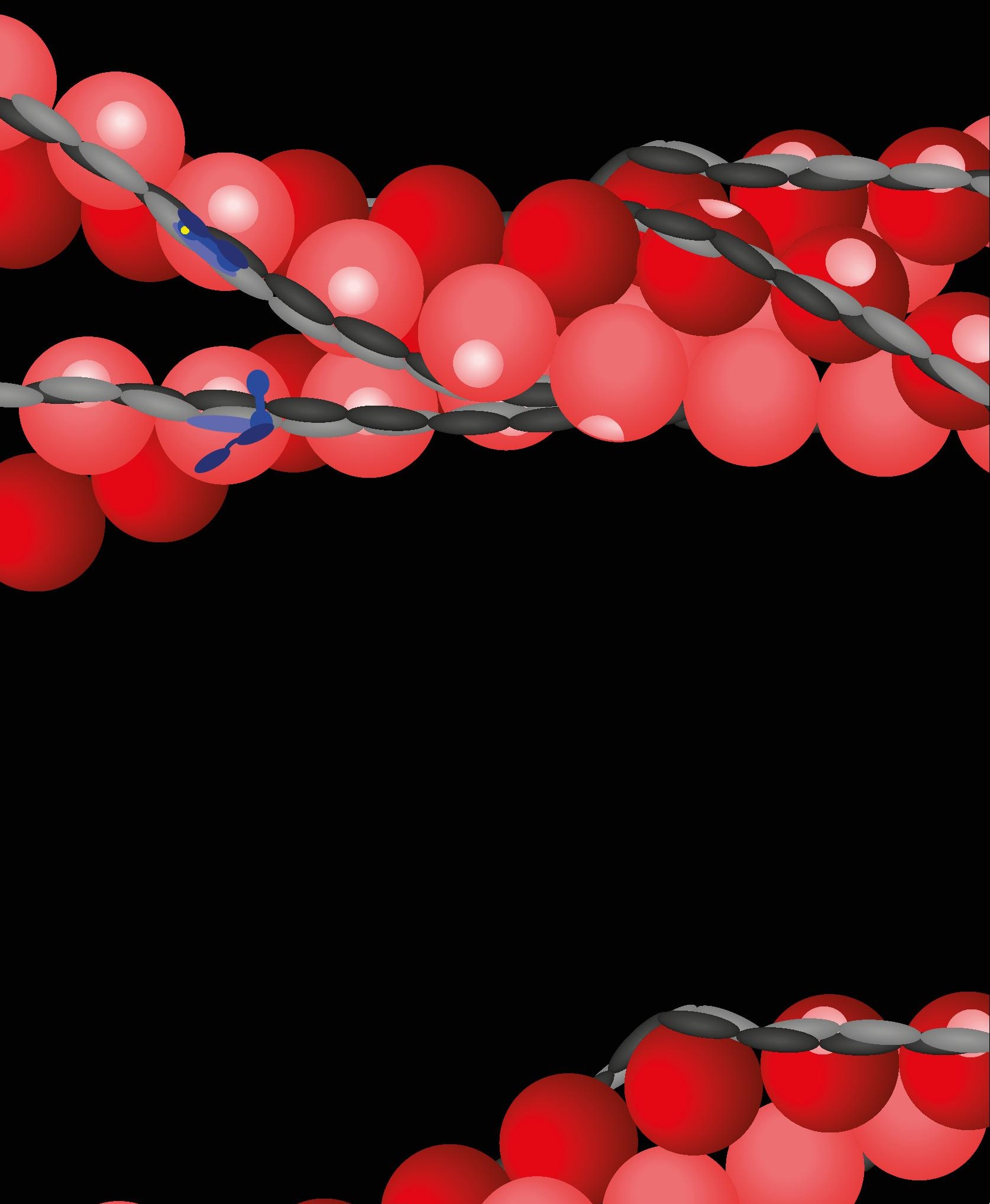

